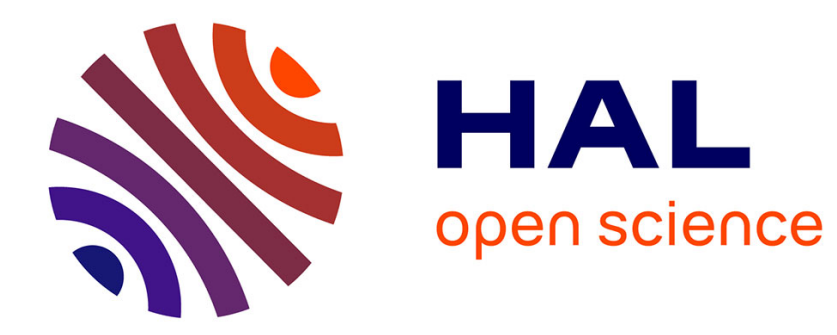

\title{
Counting connected components of a semi-algebraic set in subexponential time
}

\author{
Dima Grigoriev, Nicolai Vorobjov
}

\section{To cite this version:}

Dima Grigoriev, Nicolai Vorobjov. Counting connected components of a semi-algebraic set in subexponential time. Computational Complexity, 1992. hal-03052999

\section{HAL Id: hal-03052999 \\ https://hal.science/hal-03052999}

Submitted on 10 Dec 2020

HAL is a multi-disciplinary open access archive for the deposit and dissemination of scientific research documents, whether they are published or not. The documents may come from teaching and research institutions in France or abroad, or from public or private research centers.
L'archive ouverte pluridisciplinaire $\mathbf{H A L}$, est destinée au dépôt et à la diffusion de documents scientifiques de niveau recherche, publiés ou non, émanant des établissements d'enseignement et de recherche français ou étrangers, des laboratoires publics ou privés. 
comput complexity 2 (1992), 133-186

\title{
COUNTING CONNECTED COMPONENTS OF A SEMIALGEBRAIC SET IN SUBEXPONENTIAL TIME
}

\author{
D. YU. GRIGOR'Ev AND N. N. Vorobjov, JR.
}

\begin{abstract}
Let a semialgebraic set be given by a quantifier-free formula of the first-order theory of real closed fields with $k$ atomic subformulae of the type $f_{i} \geq 0$ for $1 \leq i \leq k$, where the polynomials $f_{i} \in \mathbb{Z}\left[X_{1}, \ldots, X_{n}\right]$ have degrees $\operatorname{deg}\left(f_{i}\right)<d$ and the absolute value of each (integer) coefficient of $f_{i}$ is at most $2^{M}$. An algorithm is exhibited which counts the number of connected components of the semialgebraic set in time $\left(M(k d)^{n^{20}}\right)^{O(1)}$. Moreover, the algorithm allows us to determine whether any pair of points from the set are situated in the same connected component.
\end{abstract}

Subject classifications. $68 \mathrm{C} 25$

\section{Introduction}

In the present paper a subexponential-time algorithm is designed which finds the number of connected components of a semialgebraic set given by a quantifierfree formula of the first-order theory of real closed fields (for a rather wide class of real closed fields, see Grigor'ev \& Vorobjov 1988, Grigor'ev 1988). Moreover, the algorithm decides, for any two points from the semialgebraic set, whether they belong to the same connected component.

Decidability of the mentioned problems follows from the quantifier elimination method in the first-order theory of real closed fields, described for the first time in Tarski (1951). However, the complexity bound of this method is nonelementary, and in particular one cannot estimate it by any finite iteration of the exponential function. In Collins (1975) (see also Wüthrich 1976) the construction of a cylindrical algebraic decomposition is proposed, which allows one to solve these problems in exponential time.

For an arbitrary ordered field $F$ we denote by $\tilde{F} \supset F$ its uniquely defined real closure (see, e.g., Lang 1965). In the following we consider input polynomials over the ordered ring $\mathbf{Z}_{m}=\mathbf{Z}\left[\delta_{1}, \ldots, \delta_{m}\right] \subset \mathbf{Q}_{m}=\mathbf{Q}\left(\delta_{1}, \ldots, \delta_{m}\right)$, where 
$\delta_{1}, \ldots, \delta_{m}$ are algebraically independent elements over $Q$ and the ordering in the field $\mathrm{Q}_{m}$ is defined as follows. The element $\delta_{1}$ is infinitesimal with respect to $\mathbf{Q}$ (i.e., $0<\delta_{1}<a$ for any rational number $0<a \in \mathbb{Q}$ ), and for each $1 \leq i<m$ the element $\delta_{i+1}>0$ is infinitesimal with respect to the field $Q_{i}$.

Thus, let a quantifier-free formula $\Xi$ of the first-order theory of real closed fields be given as input, where $\Xi$ contains atomic subformulae of the form $f_{i} \geq 0$ for $1 \leq i \leq k$, with $f_{i} \in \mathbb{Z}_{m}\left[X_{1}, \ldots, X_{n}\right]$. Any rational function $g \in \mathbb{Q}_{m}\left(Y_{1}, \ldots, Y_{s}\right)$ can be represented as $g=g_{1} / g_{2}$, where the polynomials $g_{1}, g_{2} \in \mathbf{Z}_{m}\left[Y_{1}, \ldots, Y_{s}\right]$ are relatively prime. Denote by $l(g)$ the maximum of the bit-lengths of the (integer) coefficients of the polynomials $g_{1}, g_{2}$ (in variables $\left.Y_{1}, \ldots, Y_{s}, \delta_{1}, \ldots, \delta_{m}\right)$. We will assume that the following bounds are valid:

$$
\operatorname{deg}_{X_{1}, \ldots, X_{n}}\left(f_{i}\right)<d, \operatorname{deg}_{\delta_{1}, \ldots, \delta_{m}}\left(f_{i}\right)<d_{0}, l\left(f_{i}\right) \leq M
$$

for $1 \leq i \leq k$, where $d, d_{0}, M$ are integers. Note that the bit-length of the formula $\Xi$ can be estimated by the value $\mathcal{L}=k M d^{n} d_{0}^{m}$ (see Chistov \& Grigor'ev 1983, Grigor'ev 1986).

In the case $m=0$, i.e., for polynomials with integer coefficients, the algorithms from Collins (1975) and Wüthrich (1976) allow one to produce the connected components (in particular to solve the problems considered in the present paper) within time $M^{O(1)}(k d)^{2^{O(n)}}$.

We use the notation $h_{1} \leq \mathcal{P}\left(h_{2}, \ldots, h_{t}\right)$ for the functions $h_{1}>0, \ldots, h_{t}>0$ if, for suitable integers $c, \gamma$, the inequality $h_{1} \leq c\left(h_{2} \cdot \ldots \cdot h_{t}\right)^{\gamma}$ is satisfied.

Recall that a semialgebraic set (in $F^{n}$ where $F$ is a real closed field) is a set $\{\Pi\} \subset F^{n}$ of all points satisfying a certain quantifier-free formula $\Pi$ of the first-order theory of the field $F$, where each atomic subformula of $\Pi$ has the form $(g \geq 0)$ for some $g \in F\left[X_{1}, \ldots X_{n}\right]$.

A semialgebraic set $\{\Xi\} \subset\left(\tilde{\mathbf{Q}}_{m}\right)^{n}$ is (uniquely) decomposable as the union of a finite number of connected components $\{\Xi\}=\bigcup_{1 \leq i \leq t}\left\{\Xi_{i}\right\}$, each of them in turn being a semialgebraic set determined by an appropriate quantifier-free formula $\Xi_{i}$ of the first-order theory of the field $\tilde{\mathbf{Q}}_{m}$ (see, e.g., Collins 1975 and Wüthrich 1976 for the field $F=\mathbf{R}$, and Tarski 1951 for an arbitrary real closed field; see also below).

In this paper we shall use the following way of representing the points $u=\left(u_{1}, \ldots, u_{n}\right) \in\left(\tilde{\mathbf{Q}}_{m}\right)^{n}$ (see Grigor'ev \& Vorobjov 1988). First, the field $\mathbf{Q}_{m}\left(u_{1}, \ldots, u_{n}\right)$ is represented as a primitive extension $\mathbf{Q}_{m}[\eta]$ of $\mathbf{Q}_{m}$ (see Lang 1965) where we are explicitly given:

(i) a minimal polynomial $\varphi(Z) \in \mathbf{Q}_{m}[Z]$ for $\eta$;

(ii) integers $0 \leq \alpha_{1}, \ldots, \alpha_{n} \leq \operatorname{deg}_{Z}(\varphi)$ such that $\eta=\sum_{1 \leq i \leq n} \alpha_{i} u_{i}$; 
(iii) elements $\beta_{i}^{(j)} \in \mathbb{Q}_{m}$ such that $u_{i}=\sum_{0 \leq j<\operatorname{deg}_{Z}(\varphi)} \beta_{i}^{(j)} \eta^{j}$.

Second, we are explicitly given a sequence of pluses, minuses and zeros that designate the signs of the derivatives of all orders $\varphi^{1}(\eta), \varphi^{(2)}(\eta), \ldots, \varphi^{(\operatorname{deg}(\varphi))}(\eta)$ of the polynomial $\varphi$ at $\eta$. Thom's lemma (see, e.g., Fitchas et al. 1988) implies that the latter condition uniquely determines the root $\eta$ of $\varphi$.

We say that a polynomial $g \in \mathbb{Z}_{m}\left[X_{1}, \ldots, X_{n}\right]$ satisfies the $\left(D, D_{0}, \mathcal{M}\right)$ bound if the following inequalities hold:

$$
\operatorname{deg}_{X_{1}, \ldots, X_{n}}(g)<D ; \operatorname{deg}_{\delta_{1}, \ldots, \delta_{m}}(g), \operatorname{deg}_{\delta_{1}, \ldots, \delta_{m}}\left(\beta_{i}^{(j)}\right)<D_{0} ; l(g), l\left(\beta_{i}^{(j)}\right) \leq \mathcal{M} .
$$

One then sees that the bit-length of the representation of the point $u$ does not exceed $\mathcal{P}\left(\mathcal{M}, D, D_{0}^{m}, n\right)$ (see Grigor'ev \& Vorobjov 1988, Grigor'ev 1988). The main purpose of the paper is to prove the following theorem (see also Vorobjov \& Grigor'ev 1988).

\section{THEOREM 1.}

(1) There is an algorithm which, for any formula $\Xi$ of the above form satisfying the bounds (1), finds the number of connected components (in particular, tests the connectedness) of the semialgebraic set $\{\Xi\} \subset\left(\tilde{Q}_{m}\right)^{n}$ in time $\mathcal{P}\left(M,\left(d_{0}(k d)^{n^{19}}\right)^{n} \leq \mathcal{L}^{O\left(\log ^{20} \mathcal{L}\right)}\right.$ (i.e., the time-bound is subexponential in $\left.\mathcal{L}\right)$.

(2) For any two points $u^{(1)}, u^{(2)} \in\{\Xi\}$ satisfying the $\left(\bar{d}, \bar{d}_{0}, \bar{M}\right)$-bound, the algorithm can test whether $u^{(1)}, u^{(2)}$ belong to the same connected component of $\{\Xi\}$ in time $\left.\mathcal{P}\left(M, \bar{M},\left(d_{0} \bar{d}_{0}(k d)^{n} \bar{d}\right)^{n^{18}}\right)^{n+m}\right)$ (i.e., subexponentially in $\mathcal{L}$ and in the bit lengths of the points $\left.u^{(1)}, u^{(2)}\right)$.

To prove this theorem we shall need some subroutines which we exhibit in the rest of this introduction (see also Grigor'ev 1986). First, we need the algorithm from Chistov \& Grigor'ev (1983) for decomposing an algebraic variety (considered over an algebraically closed field) into its irreducible components. We formulate this result here for the case of zero characteristic fields, taking into account that only ordered fields are considered below. This allows one to avoid some "swelling" in the formulae that could otherwise occur in nonseparable field extensions. Second, we need the algorithm from Grigor'ev \& Vorobjov (1988) for solving systems of polynomial inequalities. Third, we need the decision procedure for real closed fields from Grigor'ev (1988). Finally, we require an algorithm from Vorobjov (1989) for finding connected components of a semialgebraic curve in the space $\left(\tilde{\mathbf{Q}}_{m}\right)^{n}$.

We remark that we do not use here the subexponential-time quantifier elimination procedure for real closed fields (see Renegar 1989, Heintz et al. 1990c), 
of which we learned after our paper was already finished. Actually, one can deduce the subexponential bound for quantifier elimination from a reduction of the whole problem to the problem of eliminating a single quantifier (see Grigor'ev 1988); one can find the solution of the latter problem in Ben-Or et al. (1986).

We mention that in Canny (1988) a similar construction to the one described in Section 3 below is contained which allows one to count the number of connected components of a nonsingular bounded hypersurface. But in the mentioned work the proof of the correctness of the algorithm and the proof of the complexity bounds (which is technically very difficult) are absent (see Sections 4 and 5 of the present paper).

After the paper was already written, the authors learned that a similar result was obtained (involving a different method) by Heintz, Roy \& Solernó (1990a,b); see also Grigor'ev, Heintz, Roy, Solernó \& Vorobjov (1990).

Notice that the main theorem of the paper can be generalized to finding connected components of a semialgebraic set with similar complexity bounds. (This result will appear in Canny et al. 1991.)

So, assume for the time being that we are given a ground field $F=$ $\mathrm{Q}\left(T_{1}, \ldots, T_{m}\right)[\eta]$, where the elements $T_{1}, \ldots, T_{m}$ are algebraically independent over $\mathrm{Q}$ and the element $\eta$ is algebraic over the field $\mathrm{Q}\left(T_{1}, \ldots, T_{m}\right)$. Let $\varphi=$ $\sum_{0 \leq i \leq \operatorname{deg}_{Z}(\varphi)}\left(\varphi_{i}^{(1)} / \varphi^{(2)}\right) Z^{i} \in \mathbb{Q}\left(T_{1}, \ldots, T_{m}\right)[Z]$ be the minimal polynomial for $\eta$ over $\mathbb{Q}\left(T_{1}, \ldots, T_{m}\right)$ with the leading coefficient $l c_{Z}(\varphi)=1$, where the polynomials $\varphi_{i}^{(1)}, \varphi^{(2)} \in \mathbb{Z}\left[T_{1}, \ldots, T_{m}\right]$ and $\operatorname{deg}\left(\varphi^{(2)}\right)$ is the least possible. Every polynomial $f \in F\left[X_{1}, \ldots, X_{n}\right]$ can be uniquely represented in the form

$$
f=\sum_{0 \leq i<\operatorname{deg}_{Z}(\varphi) ; i_{1}, \ldots, i_{n}}\left(a_{i, i_{1}, \ldots, i_{n}} / b\right) \eta^{i} X_{1}^{i_{1}} \cdot \ldots \cdot X_{n}^{i_{n}}
$$

where the polynomials $a_{i, i_{1}, \ldots, i_{n}}, b \in \mathbf{Z}\left[T_{1}, \ldots, T_{m}\right]$ and $\operatorname{deg}(b)$ is the least possible. Denote the degree

$$
\operatorname{deg}_{T_{1}, \ldots, T_{m}}(f)=\max _{i, i_{1}, \ldots, i_{n}}\left\{\operatorname{deg}_{T_{1}, \ldots, T_{m}}\left(a_{i, i_{1}, \ldots, i_{n}}\right), \operatorname{deg}_{T_{1}, \ldots, T_{m}}(b)\right\}
$$

and the bit length of the coefficients $l(f)=\max _{i, i_{1}, \ldots, i_{n}}\left\{l\left(a_{i, i_{1}, \ldots, i_{n}}\right), l(f)\right\}$ (see above).

Let an input system of equations $f_{1}=\cdots=f_{k}=0$ be given, where the polynomials $f_{1}, \ldots, f_{k} \in F\left[X_{1}, \ldots, X_{n}\right]$ satisfy the following bounds: $\operatorname{deg}_{x_{1}, \ldots, x_{n}}\left(f_{i}\right)<d, \operatorname{deg}_{T_{1}, \ldots, T_{m}}\left(f_{i}\right)<d_{0}, \operatorname{deg}_{T_{1}, \ldots, T_{m}}(\varphi)<d_{0}, \operatorname{deg}_{Z}(\varphi)<d_{1}$, $l\left(f_{i}\right) \leq M, l(\varphi) \leq M$ for $1 \leq i \leq k$. For the size $\mathcal{L}_{1}$ of the system in Proposition 1 we use the value $k d^{n} d_{1} d_{0}^{m} M$. 
The variety $\mathcal{W} \subset \bar{F}^{n}$ of all roots (defined over the algebraic closure $\bar{F}$ of the field $F$ ) of the system $f_{1}=\cdots=f_{k}=0$ is representable as the union of its components $\mathcal{W}=\bigcup_{\alpha} W_{\alpha}$ which are defined and irreducible over the field $F$ (all necessary notions from algebraic geometry can be found in Shafarevich 1974). The algorithm from Proposition 1 finds the components $W_{\alpha}$ and outputs every $W_{\alpha}$ in the following two ways: by its generic point (see below), and by a system of algebraic equations such that $W_{\alpha}$ coincides with the variety of all the roots of this system.

Let $W \subset \bar{F}^{n}$ be a closed variety of dimension $\operatorname{dim} W=n-n_{1}$ defined and irreducible over $F$. Denote by $t_{1}, \ldots, t_{n-n_{1}}$ some algebraically independent elements over $F$. A generic point of the variety $W$ can be given by the field isomorphism

$$
F\left(t_{1}, \ldots, t_{n-n_{1}}\right)[\theta] \simeq F\left(X_{1}, \ldots, X_{n}\right)=F(W),
$$

where the element $\theta$ is algebraic over the field $F\left(t_{1}, \ldots, t_{n-n_{1}}\right)$. Denote by $\varphi(Z) \in F\left(t_{1}, \ldots, t_{n-n_{1}}\right)[Z]$ the minimal polynomial of $\theta$ over $F\left(t_{1}, \ldots, t_{n-n_{1}}\right)$ with leading coefficient $l c_{Z}(\varphi)=1$. The elements $X_{1}, \ldots, X_{n}$ are considered here as rational (coordinate) functions on the variety $W$. Under the isomorphism (*) we have $t_{i} \rightarrow x_{j_{i}}$ for suitable $1 \leq j_{1}<\ldots<j_{n-n_{1}} \leq n$, where $1 \leq i \leq n-n_{1}$. The element $\theta$ is the image under the isomorphism $(*)$ of an appropriate linear function $\sum_{1 \leq i \leq n} \lambda_{i} X_{i}$, where $\lambda_{1}, \ldots, \lambda_{n}$ are integers. The algorithm from Proposition 1 represents the isomorphism $(*)$ by the integers $\lambda_{1}, \ldots, \lambda_{n}$ and also by the images of the coordinate functions $X_{1}, \ldots, X_{n}$ in the field $F\left(t_{1}, \ldots, t_{n-n_{1}}\right)[\theta]$. In the formulation of Proposition 1 we sometimes identify a rational function with its image under the isomorphism.

Proposition 1. (Chistov \& Grigor'ev 1983, Grigor'EV 1986.) An algorithm can be designed which produces a generic point of every component $W_{\alpha}$ and constructs a certain family of polynomials $\psi_{\alpha}^{(1)}, \ldots, \psi_{\alpha}^{(N)} \in F\left[X_{1}, \ldots\right.$, $\left.X_{n}\right]$ such that $W_{\alpha}$ coincides with the variety of all the roots of the system $\psi_{\alpha}^{(1)}=\ldots=\psi_{\alpha}^{(N)}=0$. Let $n-n_{1}=\operatorname{dim} W_{\alpha}, \theta_{\alpha}=\theta, \varphi_{\alpha}=\varphi($ see $(*))$. Then $\operatorname{deg}_{Z}\left(\varphi_{\alpha}\right) \leq \operatorname{deg}\left(W_{\alpha}\right) \leq d^{n_{1}} ;$ and for all $1 \leq j \leq n$ and $1 \leq s \leq N$ we have

$$
\begin{array}{r}
\operatorname{deg}_{T_{1}, \ldots, T_{m}, t_{1}, \ldots, t_{n-n_{1}}}\left(\varphi_{\alpha}\right), \operatorname{deg}_{T_{1}, \ldots, T_{m}, t_{1}, \ldots, t_{n-n_{1}}}\left(X_{j}\right), \operatorname{deg}_{T_{1}, \ldots, T_{m}}\left(\psi_{\alpha}^{(s)}\right) \leq \\
d_{0} \mathcal{P}\left(d^{n_{1}}, d_{1}\right),
\end{array}
$$

and $\left.\operatorname{deg}_{X_{1}, \ldots, X_{n}}\left(\psi_{\alpha}^{(s)}\right)\right) \leq d^{2 n_{1}}$. The number of equations $N \leq n_{1}^{2} d^{4 n_{1}}$. Furthermore, $l\left(\varphi_{\alpha}\right), l\left(X_{j}\right) \leq\left(M+(n+m) d_{0}\right) \mathcal{P}\left(d^{n_{1}}, d_{1}\right)$, and

$$
l\left(\psi_{\alpha}^{(s)}\right) \leq\left(M+n d_{0}\right) \mathcal{P}\left(d^{n}, d_{1}\right) .
$$


Finally, the total running time of the algorithm can be bounded by

$$
\mathcal{P}\left(M,\left(d^{n} d_{1} d_{0}\right)^{n+m}, k\right) .
$$

Obviously, the latter value does not exceed $\mathcal{P}\left(\mathcal{L}_{1}^{\log \mathcal{L}_{1}}\right)$, in other words, is subexponential in the size $\mathcal{L}_{1}$.

We proceed to formulate the result from Grigor'ev \& Vorobjov (1988) on solving systems of polynomial inequalities over real closed fields. To do this we need to generalize the notion of a connected component of a semialgebraic set from the field $\mathbf{R}$ to arbitrary real closed fields. It is well known (Tarski 1951) that all real closed fields are equivalent in an elementary way. This means that if $K_{1}, K_{2}$ are real closed fields where $K_{1} \subset K_{2}$ and $\Pi$ is any closed formula (without free variables) from the language of the first order theory of the field $K_{1}$, then the truth values of $\Pi$ in the fields $K_{1}$ and $K_{2}$ coincide. In this paper we refer to this statement as the "transfer principle."

Now we shall demonstrate how the transfer principle can work and also show (a known fact) that any semialgebraic set over a real closed field $K$ can be represented uniquely as a union of its connected components, each in turn being a semialgebraic set. Consider a semialgebraic set $W=\{\Pi\} \subset K^{n}$, determined by a quantifier-free formula $\Pi$ of the first-order theory of real closed fields (henceforth we shall omit the term "first-order") with atomic subformulas of the kind $(f \geq 0)$ for some $f \in K\left[X_{1}, \ldots, X_{n}\right]$. By the format of the formula $\Pi$ we shall mean the sum of the number of its variables, the number of atomic subformulas and the degrees of the polynomials $f$.

In the case of the field $K=\mathbb{R}$ the set $W$ is uniquely representable as a union of its connected components $W=\bigcup_{i} W_{i}$, where every $W_{i}$ is in turn a semialgebraic set (and connected in the Euclidean topology). From the papers Collins (1975) and Wüthrich (1976) one can deduce the existence of a function $H$ such that if the format of formula $\Pi$ is less than $\mathcal{N}$ then the number of the components $W_{i}$ is less than $H(\mathcal{N})$. Moreover, one can find quantifier-free formulas $\Pi_{i}$ of the theory of real closed fields, each having format less than $H(\mathcal{N})$, such that $W_{i}=\left\{\Pi_{i}\right\}$. Indeed, the algorithms from Collins (1975) and Wüthrich (1976) allow one to produce a cylindrical algebraic decomposition of a semialgebraic set and, as a corollary, to produce its decomposition into connected components. For a given format $\mathcal{N}$ of an initial formula (with symbolic coefficients) each of the two algorithms can be represented as a rooted tree (directed outward from the root) having vertices either with out-degree one or out-degree three. The root corresponds to the initial formula; each vertex of the tree with out-degree one corresponds to an arithmetic operation; 
and finally, each vertex with out-degree three corresponds to a polynomial. The computation for an arbitrary initial formula, with the specified coefficients substituted instead of symbolic ones, proceeds along a relevant path of the tree starting from the root. It performs the corresponding arithmetic operation in a vertex with out-degree one, and branches at a vertex with out-degree three according to the sign of the corresponding polynomial. This representation as a tree provides the desired function $H$.

Thus, for a given $\mathcal{N}$, one can produce a formula $\Omega_{\mathcal{N}}$ of the theory of real closed fields (for the case of the field $K=\mathrm{R}$ ) expressing the existence of a decomposition of any semialgebraic set $W=\{\Pi\}$, where the format of $\Pi$ is less than $\mathcal{N}$, into its connected components $W=\bigcup_{i}\left\{\Pi_{i}\right\}$ such that the format of every $\Pi_{i}$ and the number of them are all less than $H(\mathcal{N})$. Moreover, the formula $\Omega_{\mathcal{N}}$ states that for each pair of indices $i \neq j$ the components $\left\{\Pi_{i}\right\}$ and $\left\{\Pi_{j}\right\}$ are "separated," i.e., the following formula of the theory of real closed fields is valid:

$$
\forall\left(a_{1}, \ldots, a_{n}\right) \in\left\{\Pi_{i}\right\} \exists z>0 \forall\left(b_{1}, \ldots, b_{n}\right) \in\left\{\Pi_{j}\right\}\left(\sum_{1 \leq l \leq n}\left(a_{l}-b_{l}\right)^{2} \geq z\right) .
$$

Furthermore, the formula $\Omega_{\mathcal{N}}$ claims the "connectedness" of every $\left\{\Pi_{i}\right\}$. This means that there do not exist two "separated" semialgebraic subsets of $\left\{\Pi_{i}\right\}$, each determined by a quantifier-free formula of the theory of real closed fields with format less than $H(H(\mathcal{N}))$.

In addition, for given $\mathcal{N}, \mathcal{M}$ one can prove (for the case of the field $K=\mathbf{R}$ ) a formula $\Omega_{\mathcal{N}, \mathcal{M}}$ of the theory of real closed fields expressing the following. Suppose $\{\Pi\}$ (where the format of $\Pi$ is less than $\mathcal{N}$ ) can be represented as the union of more than one and fewer than $\mathcal{M}$ pairwise "separated" semialgebraic sets, each being determined by a quantifier-free formula of the theory of real closed fields of format less than $\mathcal{M}$. Then $\{\Pi\}$ can be represented as the union of more than one and less than $H(\mathcal{N})$ pairwise "separated" semialgebraic "connected" sets, each being determined by a quantifier-free formula of the theory of real closed fields of format less than $H(\mathcal{N})$.

Applying the transfer principle to all the formulas $\Omega_{\mathcal{N}}$ and $\Omega_{\mathcal{N}, \mathcal{M}}$, one concludes that any semialgebraic set (over a real closed field $K$ ) can be uniquely represented as the union of its pairwise "separated," "connected components;" moreover each component is semialgebraic and is "connected," i.e., cannot be represented as the union of a finite number of pairwise "separated" semialgebraic sets. Below we utilize the terms "connected semialgebraic set" and "connected components of a semialgebraic set" without quotation marks, since the notion of connectedness in any topology will not be considered. 
Note that a semialgebraic set is connected if it is linearly connected, i.e., each two of its points can be linked by a connected semialgebraic curve. To prove this, observe that for the case of the field $K=\mathbb{R}$ the constructions described in Collins (1975) and Wüthrich (1976) imply the following statement. For a semialgebraic set determined by a quantifier-free formula of a fixed format $N_{1}$ and for every two points belonging to one of its connected components $W_{0}$, these points can be linked by a connected semialgebraic curve lying in $W_{0}$. Moreover the curve can be given by a quantifier-free formula of a format depending only on $N_{1}$. One can write the latter statement as a formula of the theory of real closed fields, and therefore, by virtue of the transfer principle, the statement is also true for an arbitrary real closed field $K$.

Let the polynomials $f_{1}, \ldots, f_{k} \in \mathbf{Z}_{m}\left[X_{1}, \ldots, X_{n}\right]$ be given, satisfying the bounds (1). We say in this case that $f_{1}, \ldots, f_{k}$ satisfy $\left(d, d_{0}, M\right)$-bounds (see formulation of the theorem).

Following Heintz (1983) (see also Grigor'ev 1988) we use the term $\left\{f_{1}\right.$, $\left.\ldots, f_{k}\right\}$-cell to denote any nonempty semialgebraic set of the form $\left\{\Lambda_{i \in I}\left(f_{i}=\right.\right.$ $\left.0) \wedge \bigwedge_{i_{1} \in I_{1}}\left(f_{i_{1}}>0\right) \wedge \bigwedge_{i_{2} \in I_{2}}\left(f_{i_{2}}<0\right)\right\}$ where $I \cup I_{1} \cup I_{2}=\{1, \ldots, k\}$. Denote by $\mathcal{U}\left(\left\{f_{1}, \ldots, f_{k}\right\}\right)$ the partition of the space $\left(\tilde{\mathbf{Q}}_{m}\right)^{n}$ into connected components of all $\left\{f_{1}, \ldots, f_{k}\right\}$-cells (see Wüthrich 1976; also Grigor'ev 1988). A finite set $\mathcal{T} \subset\left(\tilde{Q}_{m}\right)^{n}$ is called a representative set for the family of polynomials $f_{1}, \ldots, f_{k}$ if every element of the partition $\mathcal{U}\left(\left\{f_{1}, \ldots, f_{k}\right\}\right)$ contains at least one point from $\mathcal{T}$. The following proposition was proved in Grigor'ev \& Vorobjov (1988), Grigor'ev (1988), and Renegar (1989) (where the complexity bound was improved); see also Heintz et al. (1990c).

Proposition 2. One can design an algorithm which yields a representative set $\mathcal{T} \subset\left(\tilde{\mathbf{Q}}_{m}\right)^{n}$ for the family of polynomials $f_{1}, \ldots, f_{k}$ (satisfying $\left(d, d_{0}, M\right.$ ). bounds). Furthermore the algorithm, for each point from $\mathcal{T}$, specifies a cell containing this point. The running time of the algorithm does not exceed $\mathcal{P}\left(M,(k d)^{n(m+1)}, d_{0}^{m}\right)$. The number of points in $\mathcal{T}$ is at most $\mathcal{P}\left((k d)^{n}\right)$. Moreover, the algorithm represents every point from $\mathcal{T}$ in the same way as in Theorem 1 and satisfies the $\left(\mathcal{P}\left((k d)^{n}\right), d_{0} \mathcal{P}\left((k d)^{n}\right),\left(M+m d_{0} \cdot \mathcal{P}\left((k d)^{n}\right)\right)\right.$-bound.

In the following we also need a construction from Grigor'ev (1988). Consider polynomials $g_{1}, \ldots, g_{s} \in \mathbb{Q}_{m}\left[X_{1}, \ldots, X_{n}, Y_{1}, \ldots, Y_{e}\right]$. We say that a family of polynomials $h_{1}, \ldots, h_{t} \in \mathbb{Q}_{m}\left[X_{1}, \ldots, X_{n}\right]$ is thin with respect to the family $g_{1}, \ldots, g_{s}$ (and to the natural projection $\pi: \tilde{\mathbf{Q}}_{m}^{n+e} \rightarrow \tilde{\mathbf{Q}}_{m}^{n}$ ) if, for any element $U_{1} \subset \tilde{\mathbf{Q}}_{m}^{n}$ of the partition $\mathcal{U}\left(\left\{h_{1}, \ldots, h_{t}\right\}\right)$ and for any element $U_{2} \subset \tilde{\mathbb{Q}}_{m}^{n+e}$ of the partition $\mathcal{U}\left(\left\{g_{1}, \ldots, g_{s}\right\}\right)$, we have either $\pi\left(U_{2}\right) \cap U_{1}=\emptyset$ or $\pi\left(U_{2}\right) \supset U_{1}$. The 
following proposition is contained in Lemmas 12 and 14 in Grigor'ev (1988). See also Renegar (1989) and Heintz et al. (1990c).

Proposition 3. One can design an algorithm which, for a given family of polynomials $g_{1}, \ldots, g_{s} \in \mathbb{Z}_{m}\left[X_{1}, \ldots, X_{n}, Y_{1}, \ldots, Y_{e}\right]$ satisfying $\left(d, d_{0}, M\right)$-bounds, yields a family $h_{1}, \ldots, h_{t}$ which is thin with respect to this family and to the projection $\pi$. Furthermore, $h_{1}, \ldots, h_{t}$ satisfy $\left(\mathcal{P}\left((s d)^{e}\right), d_{0} \mathcal{P}\left((s d)^{e}\right),(M+\right.$ $\left.\left.m d_{0}\right) \mathcal{P}\left(n,(s d)^{e}\right)\right)$-bounds, and $t \leq(s d)^{e^{2} n}$. The running time of the algorithm does not exceed $\mathcal{P}\left(M,(s d)^{e(e n+m)}, d_{0}^{m}\right)$.

Finally, we need the following.

Proposition 4. (Vorobjov 1989, Heintz, Roy \& Solernó 1990c.) Let the polynomials $g_{1}, \ldots, g_{s}, g_{s+1}, \ldots, g_{s_{1}}, g_{s_{1}+1}, \ldots, g_{s_{2}} \in \mathbf{Z}_{m}\left[X_{1}, \ldots, X_{n}\right]$ be given, satisfying $\left(d, d_{0}, M\right)$-bounds, and suppose that any irreducible (over the field $\mathrm{Q}_{m}$ ) component $W \subset\left(\overline{\mathbf{Q}}_{m}\right)^{n}$ of the variety determined by the system of equations $g_{1}=\cdots=g_{s}=0$ such that $W \cap\left(\tilde{\mathbf{Q}}_{m}\right)^{n} \neq \emptyset$ has dimension $\operatorname{dim}_{\bar{Q}_{m}}(W) \leq 1$. Then one can find the connected components of the semialgebraic curve $\left\{g_{1}=\cdots=g_{s}=0 \wedge g_{s+1}>0 \wedge \cdots \wedge g_{s_{1}}>0 \wedge g_{s_{1}+1} \geq 0 \wedge \cdots \wedge g_{s_{2}} \geq\right.$ $0\} \subset\left(\tilde{\mathbf{Q}}_{m}\right)^{n}$ within time $\mathcal{P}\left(M,\left(s_{2} d^{n_{3}} d_{0}\right)^{n+m}\right)$. Moreover, the algorithm represents each connected component as the union of some connected semialgebraic curves of the kind $\left\{h_{1}>0 \wedge \cdots \wedge h_{t}>0 \wedge h_{t+1} \geq 0 \wedge \cdots \wedge h_{t_{1}} \geq 0\right\}$, where all the polynomials $h_{i} \in \mathbb{Z}_{m}\left[X_{1}, \ldots, X_{n}\right]$ for $1 \leq i \leq t_{1}$ satisfy $\left(\mathcal{P}\left(d^{n^{3}}\right), d_{0} \mathcal{P}\left(d^{n^{3}}\right)\right.$, $\left.\left(M+m d_{0}\right), \mathcal{P}\left(d^{n^{3}}\right)\right)$-bounds.

\section{Reduction of counting connected components to the case of a system of inequalities}

Let $K$ be an arbitrary real closed field (see, e.g., Lang 1965) and an element $\varepsilon>0$ be an infinitesimal with respect to the field $K$ (see above). Let us recall some well known facts about real closed fields. A Puiseux series (or fractionalpower series) over $K$ is a series of the form $\sum_{i \geq 0} \alpha_{i} \varepsilon^{\nu_{i} / \mu}$, where $0 \neq \alpha_{i} \in$ $K$, the integers $\nu_{0}<\nu_{1}<\ldots$ increase, and the integer $\mu \geq 1$. The field $K\left(\left(\varepsilon^{1 / \infty}\right)\right)$ consisting of all Puiseux series (with zero added) is real and closed, hence $K\left(\left(\varepsilon^{1 / \infty}\right)\right) \supset \tilde{K}(\varepsilon) \supset K(\varepsilon)$. Furthermore, the field $K[\sqrt{-1}]\left(\left(\varepsilon^{1 / \infty}\right)\right)=$ $\bar{K}\left(\left(\varepsilon^{1 / \infty}\right)\right)$ is algebraically closed.

When $\nu_{0}<0$, the element $a \in K\left(\left(\varepsilon^{1 / \infty}\right)\right)$ is called infinitely large, while if $\nu_{0}>0$ then $a$ is infinitesimal (with respect to the field $K$ ). A vector $\left(a_{1}, \ldots, a_{n}\right) \in\left(K\left(\left(\varepsilon^{1 / \infty}\right)\right)\right)^{n}$ is called $K$-finite if each of its coordinates $a_{i}$ 
$(1 \leq i \leq n)$ is not infinitely large. The standard part $s t(a) \in K$ of any $K$-finite element $a \in K\left(\left(\varepsilon^{1 / \infty}\right)\right)$ is definable (see Grigor'ev \& Vorobjov 1988); namely, st $(a)=d_{0}$ when $\nu_{0}=0$ and $\operatorname{st}(a)=0$ when $\nu_{0}>0$. Similarly, one can define the standard part of a Puiseux series from $\bar{K}\left(\left(\varepsilon^{1 / \infty}\right)\right)$. The standard part of a $K$-finite vector $\left(a_{1}, \ldots, a_{n}\right) \in\left(K\left(\left(\varepsilon^{1 / \infty}\right)\right)\right)^{n}$ is defined componentwise: $s t\left(a_{1}, \ldots, a_{n}\right)=\left(s t\left(a_{1}\right), \ldots, s t\left(a_{n}\right)\right)$. For a set $W \subset\left(K\left(\left(\varepsilon^{1 / \infty}\right)\right)\right)^{n}$ let $s t(W)=\{s t(w): w \in W$ and $s t(w)$ is definable $\}$.

Recall that an input quantifier-free formula $\Xi$ of the theory of real closed fields is given containing $k$ atomic subformulas of the form $\left(f_{i} \geq 0\right)$ for $1 \leq$ $i \leq k$, where $f_{i} \in F\left[X_{1}, \ldots, X_{n}\right]$ (here $F$ is an ordered field; later $\mathbb{Q}_{m}$ will play the role of $F$ ). The semialgebraic set $\{\Xi\}$ coincides with the union of several $\left\{f_{1}, \ldots, f_{k}\right\}$-cells.

Let a system of inequalities (see (1))

$$
f_{1}>0, \ldots, f_{k_{1}}>0, f_{k_{1}+1} \geq 0, \ldots, f_{k} \geq 0
$$

be given, where $f_{i} \in F\left[X_{1}, \ldots, X_{n}\right]$ for $1 \leq i \leq k$. The purpose of the present section is to reduce the proof of the theorem (see the Introduction) to the design of an algorithm, satisfying the requirement of Theorem 1, that handles the special case when the system (2) plays the role of the formula $\Xi$ in Theorem 1. Because of that we shall assume for some time that an algorithm for formulae of the form (2) is already designed (its bounds will be obtained later, in Section $5)$.

Applying Theorem 1 to (2) and using Proposition 2, the algorithm produces a representative system $\mathcal{T}$ for the partition $\mathcal{U}\left(\left\{f_{1}, \ldots, f_{k}\right\}\right)$ of the space $\tilde{F}^{n}$ such that each element of the partition contains a unique point from $\mathcal{T}$ (one can assume that an element of the partition is represented by this point). For an element of the partition (or in other words, for a $\left\{f_{1}, \ldots, f_{k}\right\}$-cell containing this element) one can easily test whether it lies in $\{\Xi\}$. Namely, Proposition 2 allows one to specify the signs of the polynomials $f_{1}, \ldots, f_{k}$ for a representative point of this element of the partition, and thereby the truth values of the atomic subformulae $\left(f_{i} \geq 0\right)$ (for $\left.1 \leq i \leq k\right)$ of the formula $\Xi$. The considered $\left\{f_{1}, \ldots, f_{k}\right\}$-cell lies in $\Xi$ if, after substituting for the atomic subformulas in $\Xi$ their truth values, a true proposition is obtained.

To count the connected components of the set $\{\Xi\}$ it suffices to find out, for each pair $V_{1}, V_{2} \in \mathcal{U}\left(\left\{f_{1}, \ldots, f_{k}\right\}\right)$, whether $V_{1} \cap \bar{V}_{2} \neq \emptyset$ (here the bar denotes the closure in the topology of the space $\tilde{F}^{n}$ generated by the basis of all open balls). Indeed, consider a graph whose vertices bijectively correspond to those elements of $\mathcal{U}\left(\left\{f_{1}, \ldots, f_{k}\right\}\right)$ which lie in $\{\Xi\}$, such that there is an edge between the vertices corresponding to $V_{1}, V_{2}$ if either $V_{1} \cap \bar{V}_{2} \neq \emptyset$ or $\bar{V}_{1} \cap V_{2} \neq \emptyset$. Then 
the connected components of this graph correspond bijectively in a natural way to the connected components of the set $\{\Xi$ \} (this is obvious in the case when the field $F=\mathbb{R}$; for an arbitrary real closed field one should invoke the transfer principle).

Thus, the algorithm has to test whether $V_{1} \cap \bar{V}_{2} \neq \emptyset$. Let $V_{1}$ lie in a certain $\left\{f_{1}, \ldots, f_{k}\right\}$-cell (its connected component)

$$
\begin{aligned}
U_{1}=\left\{f_{i_{1}}\right. & =\cdots=f_{i_{l}}=0 \wedge\left(f_{j_{1}}>0\right) \wedge \cdots \wedge\left(f_{j_{l_{1}}}>0\right) \\
& \left.\wedge\left(f_{j_{l_{1}+1}}<0\right) \wedge \cdots \wedge\left(f_{j_{k-l}}<0\right)\right\} .
\end{aligned}
$$

Consider $\varepsilon_{1}>0$, an infinitesimal with respect to the field $F$, and $\varepsilon_{2}>0$, an infinitesimal with respect to the field $F\left(\varepsilon_{1}\right)$. Let $F_{1}$ and $F_{2}$ denote the fields $\tilde{F}\left(\varepsilon_{1}\right)$ and $\tilde{F}\left(\varepsilon_{2}\right)$, respectively. By $s t_{2}$ we denote the standard part with respect to $\varepsilon_{2}$ and by $s t_{1}$ we denote the standard part with respect to both $\varepsilon_{1}, \varepsilon_{2}$; i.e., for $a \in F_{2}$ we have $s t_{2}(a) \in F_{1}, s t_{1}(a) \in \tilde{F}$ (provided that these standard parts of $a$ are definable). Note that an element $a-s t_{2}(a)$ is an infinitesimal with respect to the field $F_{1}$ and an element $a-s t_{1}(a)$ is an infinitesimal with respect to the field $F$. Let $V_{1}^{\left(\varepsilon_{1}, \varepsilon_{2}\right)} \subset F_{2}^{n}$ be a semialgebraic set determined in the space $F_{2}^{n}$ by the same quantifier-free formula of the theory of real closed fields (defined over the field $F$ ) as the set $V_{1}$ (we also use similar notations below).

Introduce a semialgebraic set

$$
\begin{aligned}
\mathcal{U}_{1}= & \left\{\left(-\varepsilon_{2} \leq f_{i_{1}} \leq \varepsilon_{2}\right) \wedge \cdots \wedge\left(-\varepsilon_{2} \leq f_{i_{1}} \leq \varepsilon_{2}\right) \wedge\left(f_{j_{1}} \geq \varepsilon_{2}\right)\right. \\
& \left.\wedge \cdots \wedge\left(f_{j_{l_{1}}} \geq \varepsilon_{1}\right) \wedge\left(f_{j_{l_{1}+1}} \leq-\varepsilon_{1}\right) \wedge \cdots \wedge\left(f_{j_{k-1}} \leq-\varepsilon_{1}\right)\right\} \\
\cap & \mathcal{D}_{0}\left(\varepsilon_{1}^{-1}\right) \subset F_{2}^{n}
\end{aligned}
$$

where $\mathcal{D}_{x}(r)$ denotes the closed ball $\{y:\|x-y\| \leq r\}$. Clearly, $V_{1} \subset U_{1} \subset \mathcal{U}_{1}$.

LEMMA $1 . \quad$ (a) There is a unique connected component $\mathcal{V}_{1}$ of the set $\mathcal{U}_{1}$ such that $\mathcal{V}_{1} \supset V_{1}$;

(b) $s t_{2}\left(\mathcal{V}_{1}\right) \subset V_{1}^{\left(\varepsilon_{1}, \varepsilon_{2}\right)}$;

(c) the relation $V_{1} \cap \bar{V}_{2} \neq 0$ holds if $\mathcal{V}_{1} \cap V_{2}^{\left(\varepsilon_{1}, \varepsilon_{2}\right)} \neq \emptyset$.

Proof. (a) Let $x, y \in V_{1}$. There exists a connected, bounded (i.e., lying in a certain ball $\mathcal{D}_{0}(R)$, where $R \in F$ ), closed (in the topology with the basis of all the open balls) semialgebraic curve $C \subset V_{1} \subset \tilde{F}^{n}$ containing points $x, y \in C$ (see the Introduction). Thus there exists $0<c \in F$, such that for every $z \in C$, $f_{j}(z)>c$ for $j=j_{1}, \ldots, j_{l_{1}}$ and $f_{j}(z)<c$ for $j=j_{l_{1}+1}, \ldots, j_{k-l}$. Denote 
by $C^{\left(\varepsilon_{1}, \varepsilon_{2}\right)} \subset F_{2}^{n}$ a connected bounded closed semialgebraic curve determined by the same quantifier-free formula of the theory of real closed fields as $C$ (the formulated properties of the set $C^{\left(\varepsilon_{1}, \varepsilon_{2}\right)}$ follow from the transfer principle; in particular the inclusion $C^{\left(\varepsilon_{1}, \varepsilon_{2}\right)} \subset \mathcal{D}_{0}(R)$ is valid). Also, because of the transfer principle, for each point $z_{1} \in C^{\left(\varepsilon_{1}, \varepsilon_{2}\right)}$ the inequalities $f_{j}\left(z_{1}\right)>c$ for $j=j_{1}, \ldots, j_{l_{1}}$ and $f_{j}\left(z_{1}\right)<-c$ for $j=j_{l_{1}+1}, \ldots, j_{k-l}$ hold, and furthermore $f_{i}\left(z_{1}\right)=0$ for $i=i_{i}, \ldots, i_{l}$. Hence $C^{\left(\varepsilon_{1}, \varepsilon_{2}\right)} \subset \mathcal{U}_{1}$, which means that the points $x, y$ are situated in the same connected component of the set $\mathcal{U}_{1}$.

(b) Let $x \in \mathcal{V}_{1}$. We show that $s t_{2}(x) \in V_{1}^{\left(\varepsilon_{1}, \varepsilon_{2}\right)}$ (note that $s t_{2}(x)$ is definable since $\left.x \in \mathcal{D}_{0}\left(\varepsilon_{1}^{-1}\right)\right)$. Obviously, st $t_{2}(x) \in\left\{f_{i_{1}}=\cdots=f_{i_{1}}=0 \wedge\left(f_{j_{1}} \geq \varepsilon\right) \wedge \cdots \wedge\right.$ $\left.\left(f_{j_{1}} \geq \varepsilon_{1}\right) \wedge\left(f_{j_{l_{1}+1}} \leq-\varepsilon_{1}\right) \wedge \cdots \wedge\left(f_{j_{k-1}} \leq-\varepsilon_{1}\right)\right\} \cap \mathcal{D}_{0}\left(\varepsilon_{1}^{-1}\right)=\mathcal{W} \subset F_{2}^{n}$. Note that $U_{1} \subset \mathcal{W}$. Consider an arbitrary pair of different connected components $V_{1}^{(\alpha)}$, $V_{1}^{(\beta)}$ of the cell $U_{1}$. Let $W_{1}^{(\alpha)}=V_{1}^{(\alpha)\left(\varepsilon_{1}, \varepsilon_{2}\right)} \cap \mathcal{W}$, and $W_{1}^{(\beta)}=V_{1}^{(\beta)\left(\varepsilon_{1}, \varepsilon_{2}\right)} \cap \mathcal{W}$ ' Observe that $V_{1}^{(\alpha)} \subset W_{1}^{(\alpha)}, V_{1}^{(\beta)} \subset W_{1}^{(\beta)}$. Then $W_{1}^{(\alpha)}, W_{1}^{(\beta)}$ are closed in the topology with the basis of all the open balls. Indeed, consider a point $x \in \widehat{W_{1}^{(\alpha)}}$. Then $x \in \mathcal{W}$, and taking into account that $\mathcal{W}$ is determined by the nonstrict inequalities, we have $x \in U_{1}^{\left(\varepsilon_{1}, \varepsilon_{2}\right)}$. Therefore $x \in V_{1}^{(\alpha)\left(\varepsilon_{1}, \varepsilon_{2}\right)}$, since $V_{1}^{(\alpha)\left(\varepsilon_{1}, \varepsilon_{2}\right)}$ is a connected component of a semialgebraic set $U_{1}^{\left(\varepsilon_{1}, \varepsilon_{2}\right)}$, by the transfer principle (see the Introduction) and by the fact that a connected component of a semialgebraic set is open and closed in the induced topology (also by virtue of the transfer principle). The sets $W_{1}^{(\alpha)}, W_{1}^{(\beta)}$ are determined by the systems of inequalities with coefficients from the field $F\left(\varepsilon_{1}\right)$, and they are closed and bounded. Therefore there exists $0<c \in F\left(\varepsilon_{1}\right)$ such that for any two points $x^{(1)} \in W_{1}^{(\alpha)}$ and $x^{(2)} \in W_{1}^{(\beta)}$ the distance $\left\|x^{(1)}-x^{(2)}\right\| \geq c$. We can assume that the latter inequality holds for any choice of a pair of different connected components of $U_{1}$ (here again the transfer principle is invoked).

We remark that $s t_{2}(x) \in \mathcal{W} \subset U_{1}^{\left(\varepsilon_{1}, \varepsilon_{2}\right)}$ and assume that $s t_{2}(x) \in\left(V_{1}^{\prime}\right)^{\left(\varepsilon_{1}, \varepsilon_{2}\right)}$ for a certain connected component $V_{1}^{\prime}$ of the set $U_{1}$ such that $V_{1}^{\prime}=V_{1}$. There exists a connected, closed semialgebraic curve $C_{1} \subset \mathcal{V}_{1}$, for which $x \in C_{1}$ and $C_{1} \cap V_{1} \neq \emptyset$ (see item (a) of the lemma); therefore $C_{1} \cap V_{1} \subset C_{1} \cap W_{1} \neq \emptyset$, where $W_{1}=\left(V_{1}\right)^{(\alpha)\left(\varepsilon_{1}, \varepsilon_{2}\right)} \cap \mathcal{W}$. Let $W_{1}^{\prime}=\left(V_{1}^{\prime}\right)^{\left(\varepsilon_{1}, \varepsilon_{2}\right)} \cap \mathcal{W}$. For each point $y \epsilon$ $C_{1} \subset \mathcal{V}_{1} \subset \mathcal{U}_{1}$ we have that $s t_{2}(y) \in \mathcal{W}$, and also that $\mathcal{W}=\bigcup_{\alpha}\left(V_{1}^{(\alpha)\left(\varepsilon_{1}, \varepsilon_{2}\right)} \cap \mathcal{W}\right)$ where the union ranges over all the connected components $V_{1}^{(\alpha)}$ of the set $U_{1}$. Denote by $W_{1}^{(\alpha)}(c / 3)$ the $(c / 3)$-neighborhood of the set $W_{1}^{(\alpha)}$ (observe that $W_{1}^{(\alpha)}(c / 3)$ is a semialgebraic set). Hence $C_{1} \subset \bigcup_{\alpha} W_{1}^{(\alpha)}(c / 3)$, and furthermore $c_{1} \cap W_{1} \neq \emptyset, x \in C_{1} \cap W_{1}^{\prime}(c / 3) \neq \emptyset$. This leads to a contradiction with the connectedness of $c_{1}$, taking into account the property of the element $c$. This implies $s t_{2}(x) \in V_{1}^{\left(e_{1}, \varepsilon_{2}\right)}$, i.e., item (b) of the lemma. 
(c) First, let $V_{1} \cap \bar{V}_{2} \neq \emptyset$ and let $x_{0}$ be a point in $V_{1} \cap \bar{V}_{2}$. For any point $x \in \mathcal{V}_{1} \cap \tilde{F}^{n}$ there exists an element $0<r \in F\left(\varepsilon_{1}, \varepsilon_{2}\right)$ such that $\mathcal{D}_{x}(r) \subset \mathcal{V}_{1}$, since $\mathcal{V}_{1}$ is a connected component of the set $\mathcal{U}_{1}$. Applying the latter statement to the point $x_{0}$, we get $\mathcal{V}_{1} \cap V_{2}^{\left(\varepsilon_{1}, \varepsilon_{2}\right)} \neq \emptyset$.

Conversely, let $\mathcal{V}_{1} \cap V_{2}^{\left(\varepsilon_{1}, \varepsilon_{2}\right)} \neq \emptyset$ and let $y$ be a point in $\mathcal{V}_{1} \cap V_{2}^{\left(\varepsilon_{1}, \varepsilon_{2}\right)}$. Item (b) of the lemma implies $s t_{2}(y) \in V_{1}^{\left(\varepsilon_{1}, \varepsilon_{2}\right)}$. We shall now show that $s t_{2}(y) \in$ $\overline{V_{2}^{\left(\varepsilon_{1}, \varepsilon_{2}\right)}}$. Indeed, the semialgebraic set $\overline{V_{1}^{\left(\varepsilon_{1}, \varepsilon_{2}\right)}}$ can be determined by a formula of the theory of the real closed fields over the field $\tilde{F}$. Therefore, if we assume that $s t_{2}(y) \in \overline{V_{2}^{\left(\varepsilon_{1}, \varepsilon_{2}\right)}}$, then there exists an element $0<r_{2} \in F\left(\varepsilon_{1}\right)$ such that $\mathcal{D}_{s t_{2}(y)}\left(r_{2}\right) \cap \overline{V_{2}^{\left(\varepsilon_{1}, \varepsilon_{2}\right)}} \neq \emptyset$ (here again the transfer principle is invoked). This contradicts the inclusion $y \in V_{2}^{\left(\varepsilon_{1}, \varepsilon_{2}\right)}$. Thus $V_{1}^{\left(\varepsilon_{1}, \varepsilon_{2}\right)} \cap \overline{V_{1}^{\left(\varepsilon_{1}, \varepsilon_{2}\right)}} \neq \emptyset$, whence $V_{1} \cap \bar{V}_{2} \neq \emptyset$ by virtue of the transfer principle.

Because of Lemma 1(c), to test the condition $V_{1} \cap \bar{V}_{2} \neq \emptyset$ it suffices to find out whether $\mathcal{V}_{1} \cap V_{2}^{\left(\varepsilon_{1}, \varepsilon_{2}\right)} \neq \emptyset$. Let $V_{2}$ be contained in a certain $\left\{f_{1}, \ldots, f_{k}\right\}$-cell $U_{2}$. Applying Proposition 2 and Theorem 1 to the semialgebraic set $U_{2}^{\left(\varepsilon_{1}, \varepsilon_{2}\right)} \cap \mathcal{U}_{1}$, i.e., to the corresponding system of inequalities determining this set, (see (2) above) the algorithm yields a set of representative points which correspond bijectively to the connected components of $U_{2}^{\left(\varepsilon_{1}, \varepsilon_{2}\right)} \cap \mathcal{U}_{1}$. For each representative point $x$ of the set $U_{2}^{\left(\varepsilon_{1}, \varepsilon_{2}\right)} \cap \mathcal{U}_{1}$ the algorithm tests whether $x \in V_{2}^{\left(\varepsilon_{1}, \varepsilon_{2}\right)}$, using the theorem for the cell $U_{2}^{\left(\varepsilon_{1}, \varepsilon_{2}\right)}$ and involving the representative point (produced again with the aid of Proposition 2 and Theorem 1) of the connected component $V_{2}$ of the cell $U_{2}$. Then, invoking Proposition 2 and Theorem 1 for the cell $U_{1}$, a representative point $y$ of the connected component $V_{1}$ of the cell $U_{1}$ is produced. Again using Theorem 1 for the cell $\mathcal{U}_{1}$, the algorithm tests whether $x$ and $y$ lie in the same connected component of the cell $\mathcal{U}_{1}$. The latter condition holds if $x \in \mathcal{V}_{1}$, taking into account that $y \in V_{1} \subset \mathcal{V}_{1}$ by virtue of Lemma 1 (a). So, $\mathcal{V}_{1} \cap V_{2}^{\left(\varepsilon_{1}, \varepsilon_{2}\right)} \neq \emptyset$ if, for some representative point $x$ (among those produced) of the set $U_{2}^{\left(\varepsilon_{1}, \varepsilon_{2}\right)} \cap \mathcal{U}_{1}$, the inclusions $x \in V_{2}^{\left(\varepsilon_{1}, \varepsilon_{2}\right)}$ and $x \in \mathcal{V}_{1}$ are fulfilled.

To complete the proof of Theorem 1 it remains to describe a procedure which, for a pair of points $u^{(1)}, u^{(2)} \in\{\Xi\}$, tests whether they are situated in the same connected component of the set $\{\Xi\}$. First, with the help of Proposition 2 one can find out to which $\left\{f_{1}, \ldots, f_{k}\right\}$-cells these points belong. Then applying Theorem 1 to the cell which contains $u^{(1)}$, the procedure indicates a connected component of the cell containing $u^{(1)}$ (similarly for the point $u^{(2)}$ ). Finally, applying Lemma 1 and the algorithm described above, the procedure tests whether two indicated elements of the partition $\mathcal{U}\left(\left\{f_{1}, \ldots, f_{k}\right\}\right)$ containing $u^{(1)}$ and $u^{(2)}$ respectively, lie in the same connected component of the set $\{\Xi\}$. This 
completes the reduction of the proof of Theorem 1 to the case of a system of inequalities which play the role of the quantifier-free formula $\Xi$.

\section{Reduction to the case of a bounded nonsingular hypersurface}

Now we proceed to prove the theorem in the case that a system of inequalities (2) plays the role of the formula $\Xi$. In the present section we reduce the proof of the theorem to the case of a hypersurface. Moreover, we show that it is sufficient to count only its connected components which lie in a ball of a suitable radius.

Denote by $V_{0} \subset \tilde{F}^{n}$ the semialgebraic set of all the points satisfying the system (2). Introduce a new variable $X_{0}$ and a semialgebraic set $V_{1} \subset \tilde{F}^{n+1}$ of all points satisfying the system

$$
f_{1} \geq 0, \ldots, f_{k} \geq 0, X_{0} f_{1} \cdots f_{k_{1}}=1 .
$$

There is a natural bijective correspondence between the connected components of the sets $V_{0}$ and $V_{1}$; namely, if $W_{0} \subset \tilde{F}^{n}$ is a connected component of the set $V_{0}$ then $W_{1}=\left\{\left(x_{0}, x_{1}, \ldots, x_{n}\right),\left(x_{1}, \ldots, x_{n}\right) \in W_{0} \wedge x_{0}=\right.$ $\left.\left(\left(f_{1} \cdots f_{k_{1}}\right)\left(x_{1}, \ldots, x_{n}\right)\right)^{-1}\right\}$ is the connected component of the set $V_{1}$ corresponding to $W_{0}$.

Add another new variable $X_{n+1}$ and consider the polynomials $f_{k+1}=X_{0} f_{1}$ $\cdots f_{k_{1}}-1, f_{k+2}=-f_{k+1}, f_{k+3}=X_{n+1}, f_{k+4}=-f_{k+3}$, and a system

$$
f_{1} \geq 0, \ldots, f_{k+4} \geq 0
$$

obtained from (3) by adding the inequalities $f_{k+3} \geq 0, f_{k+4} \geq 0$. Then (4) determines the semialgebraic set $V \subset \tilde{F}^{n+2}$ which is "isomorphic" to $V_{1}$ by means of a linear mapping $\pi:\left(X_{0}, \ldots, X_{n+1}\right) \mapsto\left(X_{0}, \ldots, X_{n}\right)$. Clearly $V \subset$ $\left\{X_{n+1}=0\right\}$.

Consider the semialgebraic set $\mathcal{V} \subset F_{2}^{n+2}$ determined by the following system of inequalities (here $\varepsilon_{1}, \varepsilon_{2}$ have the same meaning as in Section 1):

$$
f_{1}+\varepsilon_{1}>0, \ldots, f_{k+4}+\varepsilon_{1}>0 .
$$

For a semialgebraic set $W$ denote by $\partial W$ its boundary in the topology with the base of all open balls. Note that $\partial W$ is also a semialgebraic set.

LEMma 2. Let $\mathcal{W}$ be a connected component of the set $\mathcal{V}$. Then the boundary $\partial \mathcal{W}$ is also connected. 
Proof. Observe that $\mathcal{W}$ is a cylinder, i.e., $\mathcal{W}=U \times\left(-\varepsilon_{1}, \varepsilon_{1}\right)$ is the cartesian product of a base $U \subset F_{2}^{n+1}$ on the open segment $\left(-\varepsilon_{1}, \varepsilon_{1}\right)$. Then $U$ is connected and $\partial \mathcal{W}=\left(\partial U \times\left(-\varepsilon_{1}, \varepsilon_{1}\right)\right) \cup \bar{U}_{-\varepsilon_{1}} \cup \bar{U}_{\varepsilon_{1}}$, where $\bar{U}_{\varepsilon_{1}}=\left\{\left(x_{0}, \ldots, x_{n}, \varepsilon_{1}\right)\right.$ : $\left.\left(x_{0}, \ldots, x_{n}\right) \in \bar{U}\right\}$. Here $\bar{U}$ denotes the closure of the set $U$ in the topology with the base of all open balls. The set $\bar{U}_{-\varepsilon_{1}}$ is defined similarly. Then $\bar{U}_{\varepsilon_{1}}$, $\bar{U}_{-\varepsilon_{1}}$ are connected semialgebraic sets which are isomorphic to $\bar{U}$. Any two points $\left(x_{0}, \ldots, x_{n}, \alpha\right),\left(y_{0}, \ldots, y_{n}, \beta\right) \in \partial \mathcal{W} \backslash\left(\bar{U}_{\varepsilon_{1}} \cup \bar{U}_{-\varepsilon_{1}}\right)$ can be linked by the connected semialgebraic curves (even by the segments of the lines) lying in $\partial \mathcal{W}$ with the points $\left(x_{0}, \ldots, x_{n}, \varepsilon_{1}\right),\left(y_{0}, \ldots, y_{n}, \varepsilon_{1}\right) \in \bar{U}_{\varepsilon_{1}}$ respectively. The latter two points in turn can be linked by a connected semialgebraic curve situated in $\bar{U}_{\varepsilon_{1}}$. On the other hand, each point from $\bar{U}_{\varepsilon_{1}} \cup \bar{U}_{-\varepsilon_{1}}$ can be connected with some point from $\partial \mathcal{W} \backslash\left(\bar{U}_{\varepsilon_{1}} \cup \bar{U}_{-\varepsilon_{1}}\right)$ by a connected semialgebraic curve. Therefore $\partial \mathcal{W}$ is connected.

Let $g$ be the polynomial

$$
g=\left(f_{1}+\varepsilon_{1}\right) \cdots\left(f_{k+4}+\varepsilon_{1}\right)-\varepsilon_{2} \in F\left[\varepsilon_{1}, \varepsilon_{2}\right]\left[X_{0}, \ldots, X_{n+1}\right] .
$$

LEMMA 3. Let $S$ be a connected component of a semialgebraic set $\{g=0\} \subset$ $F_{2}^{n+2}$. Then $S$ lies in a certain connected component $\mathcal{W}_{0}$ of some open $\left\{f_{1}+\right.$ $\left.\varepsilon_{1}, \ldots, f_{k+4}+\varepsilon_{1}\right\}$-cell (in other words, a cell determined by a system of strict inequalities of the kind $(-1)^{\sigma_{1}}\left(f_{1}+\varepsilon_{1}\right)>0, \ldots,(-1)^{\sigma_{k+4}}\left(f_{k+4}+\varepsilon_{1}\right)>0$ for appropriate $\left.\sigma_{i} \in\{0,1\}, 1 \leq i \leq k+4\right)$.

Proof. Observe that for any point $x$ situated on the boundary of an arbitrary cell we have $\left(f_{1}+\varepsilon_{1}\right) \cdots\left(f_{k+4}+\varepsilon_{1}\right)(x)=0$. Therefore $S$ has no common points with the boundary of any cell, and so $S$ lies inside a certain connected component $\mathcal{W}_{0}$ of some cell. Note that this cell and each of its connected components is open in the topology with the basis of all open balls.

LEMMA 4. Let $\mathcal{W}_{1}$ be a connected component of the open cell $\mathcal{V}$ (see (5)) and let $x \in F_{1}^{n+2} \cap \partial \mathcal{W}_{1}$. Assume that $\mathcal{W}_{2}$ is a connected component of an intersection $\mathcal{W}_{1} \cap \mathcal{D}_{x}(r)$, where $0<r \in F_{1}$ is such that $x \in \partial \mathcal{W}_{2}$. Then there exists a point $y \in\{g=0\} \cap \mathcal{W}_{2}$ for which $s_{2}(y)=x$. Conversely, for any point $z \in\{g=0\} \cap \mathcal{W}_{1}$ we have $s t_{2}(z) \in \partial \mathcal{W}_{1}$, provided that $s_{2}(z)$ is definable.

Proof. Taking into account that $\mathcal{W}_{2}$ can be determined by a formula of the theory of the field $F_{1}$, we deduce that $\mathcal{W}_{2} \cap F_{1}^{n+2} \neq 0$. For any point $w \in \mathcal{W}_{2} \cap F_{1}^{n+2}$ the inequalities $F_{1} \ni\left(\left(f_{1}+\varepsilon_{1}\right) \cdots\left(f_{k+4}+\varepsilon_{1}\right)\right)(w)>c>0$ are fulfilled for a suitable $c \in F_{1}$. Because of this, $g(w)>c-\varepsilon_{2}>0$. Fix a certain 
point $w_{0} \in \mathcal{W}_{2} \cap F_{1}^{n+2}$ for which $r>\left\|x-w_{0}\right\|=r_{0}>0$. On the other hand the equation $\left(\left(f_{1}+\varepsilon_{1}\right) \cdots\left(f_{k+4}+\varepsilon_{1}\right)\right)(x)=0$ holds, and therefore $g(x)=-\varepsilon_{2}<0$.

For any $F_{2} \ni r_{2}>0$ we have $\mathcal{W}_{2} \cap \mathcal{D}_{x}\left(r_{2}\right) \neq \emptyset$ since $x \in \partial \mathcal{W}_{2}$; therefore there exists an element $0<r_{1} \in F_{2}$ such that, for any point $w_{1} \in \mathcal{W}_{2} \cap \mathcal{D}_{x}\left(r_{1}\right)$, the inequality $g\left(w_{1}\right)<0$ is true. Consider the least $r_{3}>0$ such that for some point $w_{2} \in\left(\mathcal{W}_{2} \cup \partial \mathcal{W}_{2}\right) \cap \mathcal{D}_{x}\left(r_{3}\right), g\left(w_{2}\right) \geq 0$ holds; in particular $\left\|x-w_{2}\right\|=r_{3}$. Such an element $r_{3} \leq r_{0}$ exists since the polynomial $h(w)=\|x-w\|^{2}$ has a minimum value on the nonempty closed bounded set $\left(\mathcal{W}_{2} \cup \partial \mathcal{W}_{2}\right) \cap \mathcal{D}_{x}\left(r_{0}\right) \cap\{g \geq 0\}$. Since $w_{2} \in \mathcal{W}_{2} \cup \partial \mathcal{W}_{2}$, and for any point $w \in\left(\partial \mathcal{W}_{2}\right) \cap \mathcal{D}_{x}\left(r_{0}\right)$ we have $w \in \partial \mathcal{W}_{1}$ and hence $g(w)=-\varepsilon_{2}<0$, we conclude that $w_{2} \in \mathcal{W}_{2}$.

Consider two cases. First, suppose that $s t_{2}\left(r_{3}\right)>0$. In this case there exists a point $w_{3} \in \mathcal{W}_{2} \cap \mathcal{D}_{x}\left(s t_{2}\left(r_{3}\right) / 2\right) \cap F_{1}^{n+2}$, so $g\left(w_{3}\right)>0$ (see above) and we obtain a contradiction with the choice of $r_{3}$.

In the second case, $s t_{2}\left(r_{3}\right)=0$. Assume that $g\left(w_{2}\right)>0$. Then there exists $0<r_{4} \in F_{2}$ such that $r_{4}<r_{3}$ and $\mathcal{D}_{w_{2}}\left(r_{4}\right) \subset\left(\mathcal{W}_{1} \cap \mathcal{D}_{x}(r)\right)$ (since $\left.w_{2} \in \mathcal{W}_{2} \subset \mathcal{W}_{1}\right)$, and therefore $\mathcal{D}_{w_{2}}\left(r_{4}\right) \subset \mathcal{W}_{2}$. We can assume that for any point $w_{4} \in \mathcal{D}_{w_{2}}\left(r_{4}\right)$ we have $g\left(w_{4}\right)>0$ (by choosing $r_{4}$ sufficiently small). Then any point $w_{5} \in\left(\mathcal{D}_{x}\left(r_{3}\right) \cap \mathcal{D}_{w_{2}}\left(r_{4} / 2\right)\right) \backslash \partial \mathcal{D}_{x}\left(r_{3}\right)$ satisfies $g\left(w_{5}\right)>0$, and we get a contradiction with the choice of $r_{3}$. Therefore $g\left(w_{2}\right)=0$, and this proves the first statement of the lemma if we take $y=w_{2}$.

To prove the second statement, observe that $\left(f_{1}+\varepsilon_{1}\right) \cdots\left(f_{k+4}+\varepsilon_{1}\right)$ and $\left(s t_{2}(z)\right)=s t_{2}(g(z))=0$. Thus there exists an element $0<r_{5} \in F_{1}$ such that $\mathcal{D}_{s t_{2}(z)}\left(r_{5}\right) \cap \mathcal{W}_{1}=\emptyset$; but on the other hand, $z \in \mathcal{D}_{s t_{2}(z)}\left(r_{5}\right) \cap \mathcal{W}_{1}$. This contradiction completes the proof of the lemma.

Let $S \subset F_{2}^{n+2}$ be a connected component of the variety $\{g=0\}$. Furthermore, let $S \subset \mathcal{W}_{1}$, where $\mathcal{W}_{1}$ is a certain connected component of the open set $\mathcal{V}$ (see Lemma 3 ). Lemma 2 implies connectedness of the boundary $\partial \mathcal{W}_{1}$.

Alexander's duality principle (Dold 1972) implies that $S$ decomposes a set $F_{2}^{n+2} \backslash S$ into two connected components (here we also use the fact that zero is not a critical value of the polynomial $g$, by Lemma 4 of Grigor'ev \& Vorobjov (1988), therefore $S$ is a nonsingular hypersurface). Note that here again the transfer principle is invoked. Thus it is reasonable to say about any two points from $F_{2}^{n+2} \backslash S$ either that they are situated on the same side of $S$, or on the contrary, on different sides of $S$. Note also that $S$ coincides with the boundary of every connected component of $F_{2}^{n+2} \backslash S$.

LEMma 5. (a) The whole boundary $\partial \mathcal{W}_{1}$ is situated on the same side of $S$;

(b) Any two points $x, y \in \mathcal{W}_{1} \cap F_{1}^{n+2}$ are situated on the same side of $S$. 
Proof. (a) This follows from Lemma 2, taking into account that the polynomial $g$ assumes the value $-\varepsilon_{2}<0$ everywhere on $\partial \mathcal{W}_{1}$.

(b) Since $\mathcal{W}_{1}$ can be determined by a formula of the theory of the field $F_{1}$, there exists a connected semialgebraic curve $C \subset \mathcal{W}_{1} \cap F_{1}^{n+2}$ containing $x, y \in$ $C$, determined by a formula of the theory of the field $F_{1}$ (see the Introduction). Consider the semialgebraic curve $C^{\left(\varepsilon_{2}\right)} \subset F_{2}^{n+2}$ determined by the same formula as the curve $C$. Observe that $C^{\left(\varepsilon_{2}\right)}$ is also connected (see the properties of the connected components in the Introduction). The polynomial $g$ is positive everywhere on $\mathcal{W}_{1} \cap F_{1}^{n+2}$ (see the proof of Lemma 4). In particular $g$ is positive on $C$; hence $g$ is positive on $C^{\left(\varepsilon_{2}\right)}$ by virtue of the transfer principle. Therefore $C^{\left(\varepsilon_{2}\right)}$ cannot intersect $S$.

LEMMA 6. Among all connected components of the nonsingular hypersurface $\{g=0\}$ which lie in $\mathcal{W}_{1}$, there exists exactly one containing a point $x$ whose standard part $s t_{2}(x)$ is definable.

Proof. Lemma 4 guarantees the existence of at least one connected component $S \subset \mathcal{W}_{1}$ satisfying the required properties.

Assume that there are two different connected components $S_{1}, S_{2} \subset \mathcal{W}_{1}$ of the hypersurface $\{g=0\}$, both having points with definable standard parts $s t_{2}$. Then the set $F_{2}^{n+2} \backslash\left(S_{1} \cup S_{2}\right)$ consists of three connected components by Alexander's duality principle (Dold 1972). Lemma 5(a) implies that $\partial \mathcal{W}_{1}$ is situated in one of these connected components. Then two other connected components are situated in $\mathcal{W}_{1}$, since $\partial \mathcal{W}_{1}$ is connected (see Lemma 2) and because of this the set $F_{2}^{n+2} \backslash \mathcal{W}_{1}$ is also connected. By virtue of Lemma $5(\mathrm{~b})$ at least one of these two connected components (let us denote it by $\mathcal{W}_{2}$ ) has no points in the space $F_{1}^{n+2}$. Observe that the boundary $\partial \mathcal{W}_{2}$ coincides either with $S_{1}$, with $S_{2}$, or with $S_{1} \cup S_{2}$. In particular the polynomial $g$ vanishes everywhere on $\partial \mathcal{W}_{2}$.

By the assumption, there exists a point $x \in \partial \mathcal{W}_{2}$ for which $s t_{2}(x) \in F_{1}^{n+2}$ is definable. Introduce a hypercube $\mathcal{K}$ determined by a system of inequalities $x_{i}^{(0)} \leq X_{i} \leq x_{i}^{(0)}+1,0 \leq i \leq n+1$ for appropriate $x_{0}^{(0)}, \ldots, x_{n+1}^{(0)} \in F_{1}$ such that $\mathcal{D}_{x}(1 / 3) \subset \mathcal{K}$. Then $\mathcal{W}_{2} \cap \mathcal{K} \neq \emptyset$. Consider a facet $\mathcal{E}$ of the hypercube $\mathcal{K}$ of the least possible dimension for which $\mathcal{E} \cap \mathcal{W}_{2} \neq \emptyset$ (we assume that the hypercube itself coincides with its facet of the highest dimension $n+2$ ). Denote by $\mathcal{L}_{\mathcal{E}}=\left\{X_{i_{1}}=x_{i_{1}}^{(1)} \wedge \cdots \wedge X_{i_{j}}=x_{i_{j}}^{(1)}\right\}$ the least affine subspace of $F_{1}^{n+2}$ containing $\mathcal{E}$ (obviously for each $1 \leq l \leq j$ either $x_{i_{l}}^{(1)}=x_{i_{l}}^{(0)}+1$ or $\left.x_{i_{l}}^{(1)}=x_{i_{l}}^{(0)}+1\right)$. Then the polynomial $g$ vanishes everywhere on $\partial^{\mathcal{E}}\left(\mathcal{E} \cap \mathcal{W}_{2}\right)$, where $\partial^{\mathcal{E}}$ denotes the boundary considered in the space $\mathcal{L}_{\mathcal{E}}$. Indeed, if for a 
certain point $y \in \partial^{\mathcal{E}}\left(\mathcal{E} \cap \mathcal{W}_{2}\right)$ we have $g(y) \neq 0$, then $y \in \mathcal{W}_{2}$ (taking into account that $g$ vanishes on $\partial \mathcal{W}_{2}$ ). Hence $y \notin \partial \mathcal{E}$ by virtue of the choice of $\mathcal{E}$, so we get a contradiction which proves the desired statement.

Denote by $g_{\mathcal{E}}$ the polynomial obtained from $g$ by substituting the values $x_{i_{l}}^{(1)}$ for the variables $X_{i_{e}}$ for all $1 \leq l \leq j$ (so $g_{\mathcal{E}}$ is the restriction of $g$ on $\mathcal{L}_{\mathcal{E}}$ ). Then the polynomial $g_{\mathcal{E}}$ has either a maximum nonzero value or a minimum nonzero value on the bounded closed semialgebraic set $\left(\mathcal{W}_{2} \cap \mathcal{E}\right) \cup \partial^{\mathcal{E}}\left(\mathcal{W}_{2} \cap \mathcal{E}\right)$. Indeed, if not, then taking into account that $\mathcal{W}_{2} \cap \mathcal{E}$ contains an open ball in $\mathcal{L}_{\mathcal{E}}$ it would follow that $g_{\mathcal{E}}$ vanishes identically and therefore $g$ would vanish at every point from the set $F_{1}^{n+2} \cap \mathcal{L}_{\mathcal{E}} \neq \emptyset$. This leads to a contradiction with the observation that $g$ does not vanish at any point from the set $F_{1}^{n+2}$. Let, for definiteness, $g_{\mathcal{E}}$ have a minimum value on $\left(\mathcal{W}_{2} \cap \mathcal{E}\right) \cup \partial^{\mathcal{E}}\left(\mathcal{W}_{2} \cap \mathcal{E}\right)$ different from zero at a certain point $z_{0}$ (by what we have proven above, $z_{0} \notin \partial^{\mathcal{E}}\left(\mathcal{W}_{2} \cap \mathcal{E}\right)$ ).

Any point from $\mathcal{L}_{\mathcal{E}}$ at which $g_{\mathcal{E}}$ reaches this minimum belongs to a semialgebraic set $\mathcal{W}_{3} \subset \mathcal{L}_{\mathcal{E}}$ consisting of all the points from $\mathcal{L}_{\mathcal{E}}$ satisfying the following system of equations

$$
\frac{\partial g_{\mathcal{E}}}{\partial X_{0}}=\cdots=\frac{\partial g_{\mathcal{E}}}{\partial X_{n+1}}=0
$$

On each connected component of the semialgebraic set $\mathcal{W}_{3}$ the polynomial $g_{\mathcal{E}}$ has a constant value. Taking into account that $\frac{\partial g_{\mathcal{E}}}{\partial X_{i}} \in F_{1}\left[X_{0}, \ldots, X_{n+1}\right]$ for $0 \leq i \leq n+1$ (in other words $\mathcal{W}_{3} \subset F_{2}^{n+2}$ is definable over the field $F_{1}$ ), every connected component of the set $\mathcal{W}_{3}^{\left(\varepsilon_{1}\right)} \subset \mathcal{L}_{\mathcal{E}} \cap F_{1}^{n+2}$, determined in $\mathcal{L}_{\mathcal{E}}$ by the same system (6), can be determined by a certain formula of the theory of the field $F_{1}$. The semialgebraic sets determined by the same formulas are then the connected components of the set $\mathcal{W}_{3}$ (see the Introduction). In particular, any connected component of the set $\mathcal{W}_{3}$ contains at least one point from $F_{1}^{n+2}$.

Consider a connected component $\mathcal{W}_{4}$ of the set $\mathcal{W}_{3}$ which contains the point $z_{0} \in \mathcal{W}_{4}$. We then have $\mathcal{W}_{4} \subset \mathcal{W}_{2} \cap \mathcal{E}$, since $\mathcal{W}_{4} \cap \partial^{\mathcal{E}}\left(\mathcal{W}_{2} \cap \mathcal{E}\right)=\emptyset$ because $g_{\mathcal{E}}$ has a nonzero constant value on $\mathcal{W}_{4}$. There exists a point $z_{1} \in \mathcal{W}_{4} \cap F_{1}^{n+2}$. It follows that $z_{1} \in \mathcal{W}_{2}$, and a contradiction with the relation $\mathcal{W}_{2} \cap F_{1}^{n+2}=\emptyset$ is obtained.

LEMMA 7. For any connected component $\mathcal{W}_{0}$ of the set $\mathcal{V}$ (see (5)) having at least one point $x$ whose standard part $s t_{1}(x)$ is definable, there exists a connected component $W_{0}$ of the set $V$ (see (4)) such that $W_{0} \subset \mathcal{W}_{0}$ (in the case that for every point $y \in \mathcal{W}_{0}$, the standard part $s t_{1}(y)$ is definable, such a connected component $W_{0}$ is unique; moreover $\left.s_{1}\left(\mathcal{W}_{0}\right)=W_{0}\right)$. On the other hand, for any connected component $W$ of the set $V$ there exists a unique 
connected component $\mathcal{W}$ of the set $\mathcal{V}$ which has a nonempty intersection with $W$, and the inclusion $W \subset \mathcal{W}$ holds.

PROOF. To prove the latter statement, suppose that $\mathcal{W}_{1}, \mathcal{W}_{2}$ are connected components of the set $\mathcal{V}$ and that there exists points $y_{1}, y_{2} \in \tilde{F}^{n+2}$ for which $y_{1} \in \mathcal{W}_{1} \cap W$ and $y_{2} \in \mathcal{W}_{2} \cap W$. There exists a closed connected semialgebraic curve $C \subset W \subset \dot{F}^{n+2}$ containing the points $y_{1}, y_{2} \in C$. Denote by $C^{\left(\varepsilon_{1}, \varepsilon_{2}\right)} \subset$ $\mathrm{F}_{2}^{\mathrm{n}+2}$ a semialgebraic curve, determined by the same formula of the theory of real closed fields as $C . C^{\left(\varepsilon_{1}, \varepsilon_{2}\right)}$ is also connected (see the Introduction). Moreover, for each $1 \leq i \leq k+4$ the polynomial $f_{i}$ is nonnegative everywhere on $C^{\left(\varepsilon_{1}, \varepsilon_{2}\right)}$ because of $(4)$ and the transfer principle, hence $C^{\left(\varepsilon_{1}, \varepsilon_{2}\right)} \subset \mathcal{V}$. Therefore, the semialgebraic sets $\mathcal{W}_{1} \cup C^{\left(\varepsilon_{1}, \varepsilon_{2}\right)}$ and $\mathcal{W}_{2} \cup C^{\left(\varepsilon_{1}, \varepsilon_{2}\right)} \subset \mathcal{V}$ are connected, and so $\mathcal{W}_{1}=\mathcal{W}_{2}$. The existence of the connected component $\mathcal{W}$ having a nonempty intersection with $W$ follows from the inclusion $V \subset \mathcal{V}$. The already proven uniqueness implies that $\mathcal{W} \supset W$.

To prove the first statement of the lemma, assume that a connected component $\mathcal{W}_{0}$ of the set $\mathcal{V}$ contains a point $y \in \mathcal{W}_{0}$ for which $s t_{1}(y)=\left(y_{0}, \ldots, y_{n+1}\right) \in$ $\tilde{F}^{n+2}$ is defined. Obviously, $s t_{1}(y) \in V$. Consider a hypercube $\mathcal{K}=\left\{y_{i}-1 \leq\right.$ $\left.X_{i} \leq y_{i}+1,0 \leq i \leq n+1\right\} \subset F_{2}^{n+2}$. Consider also a function $f(x)=$ $\min _{1 \leq i \leq k+4} f_{i}(x)$ and then take its maximal value $x$ on a (nonempty) closed bounded semialgebraic set $\left(\mathcal{K} \cap \mathcal{W}_{0}\right) \cup \partial\left(\mathcal{K} \cap \mathcal{W}_{c}\right)$ (which exists by virtue of the transfer principle). Observe that for any point $z \in\left(\mathcal{K} \cap \mathcal{W}_{0}\right) \cup \partial\left(\mathcal{K} \cap \mathcal{W}_{0}\right)$ such that $f(z)=x$ we have $z \notin \partial \mathcal{W}_{0}$. Indeed, for each point $y \in \mathcal{W}_{0}$ an inequality $f(y)>-\varepsilon$ is true, therefore $x>-\varepsilon_{1}$. On the other hand, for every point $z_{1} \in \partial \mathcal{W}_{0}$ we have $f\left(z_{1}\right)=-\varepsilon_{1}$.

Consider the semialgebraic set $\mathcal{U} \subset \mathcal{K}$ consisting of all points at which the function $f$ has a local maximum in $\mathcal{K}$. So, $\mathcal{U}$ can be determined by a certain formula of the theory of real closed fields with coefficients from the field $F$. Any point $z \in\left(\mathcal{K} \cap \mathcal{W}_{0}\right) \cup \partial\left(\mathcal{K} \cap \mathcal{W}_{0}\right)$, for which $f(z)=x$, belongs to $\mathcal{U}$. Since $z \notin \partial \mathcal{W}_{0}$ as shown above, $z \in \mathcal{W}_{0} \cap \mathcal{U}$. Consider a connected component $\mathcal{U}_{1}$ of the set $\mathcal{U}$ such that $\mathcal{W}_{0} \cap \mathcal{U}_{1} \neq \emptyset$. Let us show that $\mathcal{U}_{1} \subset \mathcal{W}_{0}$. Assume the contrary. Then there exists a point $z_{2} \in \mathcal{U}_{1} \cap \partial \mathcal{W}_{0} \cap \partial\left(\mathcal{K} \cap \mathcal{W}_{0}\right)$ (in order to ascertain this, consider a closed connected semialgebraic curve $C_{1} \subset \mathcal{U}_{1}$ containing some point from $\mathcal{W}_{0} \cap \mathcal{U}_{1}$ and also some point from $\mathcal{U}_{1} \backslash \mathcal{W}_{0}$; as $z_{2}$ one can take a point from $\left.\partial\left(C_{1} \cap \mathcal{W}_{0}\right) \backslash\left(C_{1} \cap \mathcal{W}_{0}\right)\right)$. Hence $f\left(z_{2}\right)=-\varepsilon_{1}$. On the other hand, for any $r>0$ there exists a point $z_{3} \in \mathcal{K} \cap \mathcal{W}_{0} \cap \mathcal{D}_{z_{2}}(r)$, so obviously $f\left(z_{3}\right)>-\varepsilon_{1}$. We get a contradiction with the condition that $f$ has a local maximum in $\mathcal{K}$ at $z_{2}$. Thus, the inclusion $\mathcal{U}_{1} \subset \mathcal{W}_{0}$ is shown.

Taking into account that $\mathcal{U}_{1}$ can be determined by a certain formula of the theory of real closed fields with coefficients from the field $F$, there exists a point 
$z_{4} \in \mathcal{U}_{1} \cap \tilde{F}^{n+2}$. Hence $z_{4} \in \mathcal{W}_{0} \subset \mathcal{V}$; therefore $z_{4} \in V$ since $f_{i}\left(z_{4}\right)+\varepsilon_{1}>0$ for $1 \leq i \leq k+4$, and because of that $f_{i}\left(z_{4}\right)=s t_{1}\left(f_{i}\left(z_{4}\right)+\varepsilon_{1}\right) \geq 0$. This implies that $\mathcal{W}_{0} \cap V \neq \emptyset$. Thus $\mathcal{W}_{0} \cap W_{0} \neq 0$ for a suitable connected component $W_{0}$ of the set $V$, and the previously proved second statement of the lemma implies the inclusion $W_{0} \subset \mathcal{W}_{0}$.

Finally, the uniqueness of $W_{0}$ in the case when the standard part $s t_{1}$ is definable for any point from $\mathcal{W}_{0}$ follows from the inclusion $s t_{1}\left(\mathcal{W}_{0}\right) \subset V$ with the aid of Lemma 1 of Grigor'ev \& Vorobjov (1988).

LEMMA 8. There exists an $R$ such that for any $R_{1} \geq R$ and every connected component $W$ of the set $V$, the intersection $W \cap \mathcal{D}_{0}\left(R_{1}\right)$ is nonempty and connected, where $R$ denotes in the case

(a) $m=0$ (i.e., $F=\mathbf{Q}$ ), an appropriate integer $R \leq \exp \left(M \cdot \mathcal{P}\left((k d)^{n}\right)\right.$ ), and in the case

(b) $m>0$, an element $R=\delta_{m}^{-s}$ for an appropriate integer $s \leq d_{0} \mathcal{P}\left((k d)^{n}\right)$.

Proof. Note that a point $x=\left(x_{0}, \ldots, x_{n+1}\right) \in\{g=0\}$ is a critical point (Hirsch 1976) for the function $\left(X_{0}, \ldots, X_{n+1}\right) \rightarrow X_{0}^{2}+\cdots+X_{n+1}^{2}=$ $\left\|\left(X_{0}, \ldots, X_{n+1}\right)\right\|^{2}$ (the square of the norm) on the hypersurface $\{g=0\}$, if the normal vectors at the point $x$ of the hypersurface $\{g=0\}$ and of the sphere $\partial \mathcal{D}_{0}(\|x\|)$ are colinear. In other words the gradient $\left(\frac{\partial g}{\partial X_{0}}, \ldots, \frac{\partial g}{\partial X_{n+1}}\right)(x)$ is colinear with the vector $x$. The latter condition is equivalent to the following system of equalities

$$
g(x)=0, \frac{\partial g}{\partial X_{j}}(x) \cdot x_{i}-\frac{\partial g}{\partial X_{i}}(x) \cdot x_{j}=0, \quad \text { for } 0 \leq i, j \leq n+1 .
$$

When $x$ is a critical point then the value $\|x\|^{2}$ of the function $\|\left(X_{0}, \ldots\right.$, $\left.X_{n+1}\right) \|^{2}$ is called a critical value. Sard's theorem (see Hirsch 1976) implies that there is only a finite number of critical values. Pick from among them all the values whose standard parts $s t_{1}$ are definable, and denote by $R^{\prime} \in \tilde{F}$ the maximum of these standard parts. As $R$ we shall take the least number larger than $\left(R^{\prime}\right)^{1 / 2}+1$ being an integer in case (a) and being of the kind $\delta_{m}^{-s}$ in case (b). First of all let us check that $R$ satisfies the bounds required in the lemma. Indeed, the norms of all points from any connected component of the semialgebraic set determined by system (7) are equal, again by virtue of Sard's theorem. Proposition 2 (see the Introduction) implies that every connected component under consideration contains a point $\left(z_{0}, \ldots, z_{n+1}\right)$ satisfying a $\left(\mathcal{P}\left((k d)^{n}\right), d_{0} \mathcal{P}\left((k d)^{n}\right),\left(M+m d_{0}\right) \mathcal{P}\left((k d)^{n}\right)\right.$-bound. The point $s t_{1}\left(z_{0}, \ldots, z_{n+1}\right) \in \tilde{F}^{n+2}$ (provided that $s t_{1}$ is definable) satisfies a bound of 
the same type and so the norm of this point satisfies the bounds required in the lemma (see Lemmas 9, 10 of Grigor'ev \& Vorobjov 1988).

One can assume without loss of generality (increasing $R$ if necessary while preserving the type of the bounds in the lemma; see Lemma 10 of Grigor'ev \& Vorobjov 1988) that $\mathcal{D}_{0}(R) \not \subset V$ (hence also $\mathcal{D}_{0}(R) \not \subset W$ ) and the intersection of $\mathcal{D}_{0}(R)$ with any connected component of the set $V$ is nonempty.

We will prove that $R$ satisfies the lemma. Let $R<R_{2} \leq R_{1}$, where $R_{1}, R_{2} \in$ $\tilde{F}$. Consider some connected component $S$ of the hypersurface $\{g=0\} \subset$ $F_{2}^{n+2}$ which contains a point $y \in S \cap \mathcal{D}_{0}\left(R_{2}\right)$. Consider the representations of the semialgebraic sets $S \cap \mathcal{D}_{0}\left(R_{1}\right)=\bigcup_{i \in I} S_{1}^{(i)}$ and $S \cap \mathcal{D}_{0}\left(R_{2}\right)=\bigcup_{j \in J} S_{2}^{(j)}$ as the unions of their connected components. Taking into account that the function $\left(X_{0}, \ldots, X_{n+1}\right) \rightarrow\left\|\left(X_{0}, \ldots, X_{n+1}\right)\right\|^{2}$ on the hypersurface $\{g=0\}$ has no critical values in the interval $\left[R_{2}^{2}, R_{1}^{2}\right]$ (Hirsch 1976), one can find a bijective correspondence $\sigma: I \rightarrow J$ such that $S_{1}^{(i)} \supset S_{2}^{(\sigma(i))}$ and $S_{1}^{(i)}$ is homeomorphic to $S_{2}^{(\sigma(i))}$. Furthermore, the intersection $S \cap\left(\mathcal{D}_{0}\left(R_{1}\right) \backslash \partial \mathcal{D}_{0}\left(R_{1}\right)\right)$ is homotopically equivalent to $S \cap \mathcal{D}_{0}\left(R_{2}\right)$; in particular, $\sigma$ provides the bijective correspondence between their connected components.

Let $\mathcal{W}$ be a connected component of the semialgebraic set $\mathcal{V}$ (see (5)), containing a point $z \in \mathcal{W}$ with definable $s t_{1}(z)$. According to Lemma 6 there is a unique connected component $S$ of the hypersurface $\{g=0\}$ such that $S \subset \mathcal{W}$ and there exists a point $y \in S$ with definable $s t_{2}(y)$. Lemma 7 implies the existence of a connected component $W \subset \mathcal{W}$ of the set $V$. By virtue of the above remark $W \cap \mathcal{D}_{0}(R) \neq \emptyset$ and $W \not \supset \mathcal{D}_{0}(R)$, and by the same token $\mathcal{W} \cap \mathcal{D}_{0}(R) \neq \emptyset$ and $\mathcal{W} \supset \mathcal{D}_{0}(R)$.

Consider the representations of the semialgebraic sets $\mathcal{W} \cap \mathcal{D}_{0}\left(R_{1}\right)=$ $\bigcup_{i \in I_{1}} \mathcal{W}_{1}^{(i)}$ and $\mathcal{W} \cap \mathcal{D}_{0}\left(R_{2}\right)=\bigcup_{j \in J_{1}} \mathcal{W}_{2}^{(j)}$ into the unions of their connected components. Fix a component $\mathcal{W}_{2}^{(j)}$ and pick out a point $w_{2} \in \partial \mathcal{W}_{2}^{(j)} \cap$ $\left(\mathcal{D}_{0}\left(R_{2}\right) \backslash \partial \mathcal{D}_{0}\left(R_{2}\right)\right) \cap F_{1}^{n+2}$. Such a point exists since each of the following sets can be determined by a system of inequalities with coefficients from the field $F_{1}: \mathcal{V}, \mathcal{W}, \mathcal{W} \cap \mathcal{D}_{0}\left(R_{2}\right)$ and $\mathcal{W}_{2}^{(j)}$. Lemma 4 ensures the existence of a point $y_{2} \in\{g=0\} \cap \mathcal{W}_{2}^{(j)}$ such that $s t_{2}\left(y_{2}\right)=w_{2}$. Then $y_{2} \in S$ because of Lemma 6. Since $F_{1} \ni\left\|w_{2}\right\|<R_{2} \in F_{1}$, it follows that $\left\|y_{2}\right\|<R_{2}$, i.e., $y_{2} \in \mathcal{D}_{0}\left(R_{2}\right) \backslash \partial \mathcal{D}_{0}\left(R_{2}\right)$

Our purpose is to prove that there are suitable bijective mappings $\pi: I_{1} \rightarrow$ $J_{1}$ and $\rho: I_{1} \rightarrow I$ of the sets of indices for which the following commutative 
diagram of inclusions is valid (for every $i \in I_{1}$ ):

$$
\bigcup_{1}^{\mathcal{W}_{1}^{(i)} \supset \mathcal{W}_{2}^{(\pi(i))}} \cup_{S_{1}^{(\rho(i))}}^{\bigcup_{S_{2}^{(\sigma(\rho(i)))}}}
$$

(for $S$ we utilize the notation introduced above).

For an appropriate index $j_{2} \in J$ we have $y_{2} \in S_{2}^{\left(j_{2}\right)}$. Therefore $S_{2}^{\left(j_{2}\right)} \subset \mathcal{W}_{2}^{(j)}$. Now let $y_{1}$ be a point in $\mathcal{W}_{2}^{(l)} \cap\{g=0\}$ for some $J_{1} \ni l \neq j$. Lemma 6 implies that $y_{1} \in S_{2}^{\left(j_{1}\right)}$ for an appropriate index $j_{1} \in J$. We show that $j_{1} \neq j_{2}$. Assume the contrary. Then $y_{1}, y_{2} \in S_{2}^{\left(j_{1}\right)} \subset \mathcal{W} \cap \mathcal{D}_{0}\left(R_{2}\right)$. Therefore $y_{1}, y_{2}$ lie in the same connected component of the set $\mathcal{W} \cap \mathcal{D}_{0}\left(R_{2}\right)$, since $S_{2}^{\left(j_{1}\right)}$ is connected. The obtained contradiction shows that $j_{1} \neq j_{2}$.

Thus, we have shown that to any component $\mathcal{W}_{2}^{(j)}$ corresponds a nonempty subset of indices $J^{(j)} \subset J$ such that $\mathcal{W}_{2}^{(j)} \supset S_{2}^{\left(j_{2}\right)}$ for all $j_{2} \in J^{(j)}$, and moreover the family $\left\{J^{(j)}\right\}_{j \in J_{1}}$ forms a partition of the set $J$. Similarly, there is a partition $\left\{I^{(i)}\right\}_{i \in I_{1}}$ of the set of indices $I$ (into nonempty subsets) such that for every $i \in I_{1}$ we have $\mathcal{W}_{1}^{(i)} \supset S_{1}^{\left(i_{1}\right)}$ for all $i_{1} \in I^{(i)}$.

We will prove that each of the sets $I^{(i)}$ (as well as $J^{(j)}$ ) consists of a single element. Assuming the contrary, we follow the proof and the notation from Lemma 6. Let $S_{1}^{\left(l_{1}\right)} \subset \mathcal{W}_{1}^{(i)}, S_{1}^{\left(l_{2}\right)} \subset W_{1}^{(i)}$ for some different $l_{1}, l_{2} \in I$. Consider an intersection $\mathcal{W}_{1}^{(i)} \cap\left(\mathcal{D}_{0}\left(R_{1}\right) \backslash \partial \mathcal{D}_{0}\left(R_{1}\right)\right)$, which is connected since $\mathcal{W}_{1}^{(i)}$ is open in $\mathcal{D}_{0}\left(R_{1}\right)$. Furthermore (see above), there exists a bijective correspondence between the connected components of the sets $S \cap\left(\mathcal{D}_{0}\left(R_{1}\right) \backslash \partial \mathcal{D}_{0}\left(R_{1}\right)\right)$ and $S \cap \mathcal{D}_{0}\left(R_{1}\right)$, hence the sets $S_{1}=S_{1}^{\left(l_{1}\right)} \backslash \partial \mathcal{D}_{0}\left(R_{1}\right)$ and $S_{2}=S_{1}^{\left(l_{2}\right)} \backslash \partial \mathcal{D}_{0}\left(R_{1}\right)$ are connected. In the proof of Lemma 6 , Alexander's duality principle was applied to the space $F_{2}^{n+2}$; here we apply it to the open ball $\mathcal{D}_{0}\left(R_{1}\right) \backslash \partial \mathcal{D}_{0}\left(R_{1}\right)$, using the homeomorphism between the space $\mathbb{R}^{n}$ and an open ball in it, and invoking the transfer principle. Consider an open subset $\mathcal{W}_{2} \subset \mathcal{W}_{1}^{(i)}$ such that $\mathcal{W}_{2} \cap F_{1}^{n+2}=\emptyset$. Note moreover that $\partial \mathcal{W}_{2} \cap\left(\mathcal{D}_{0}\left(R_{1}\right) \backslash \partial \mathcal{D}_{0}\left(R_{1}\right)\right)$ coincides with either $S_{1}, S_{2}$, or $S_{1} \cup S_{2}$ (similar to the proof of Lemma 6). There exists a point $x \in \partial \mathcal{W}_{2} \cap\left(\mathcal{D}_{0}\left(R_{1}-1 / 2\right) \backslash \partial \mathcal{D}_{0}\left(R_{1}-1 / 2\right)\right)$, taking into account that $S_{1} \cap\left(\mathcal{D}_{0}\left(R_{1}-1 / 2\right) \backslash \partial \mathcal{D}_{0}\left(R_{1}-1 / 2\right)\right)$ is homeomorphic to $S_{1} \cap\left(\mathcal{D}_{0}\left(R_{1}\right) \backslash \partial \mathcal{D}_{0}\left(R_{1}\right)\right)$ (similarly for $S_{2}$ ) since $R>\left(R^{\prime}\right)^{1 / 2}+1$ (see the beginning of the proof of the lemma). Introduce a hypercube $\mathcal{K} \subset \mathcal{D}_{0}\left(R_{1}\right) \backslash \partial \mathcal{D}_{0}\left(R_{1}\right)$ determined over the field $F_{1}$ with a certain length of its edge $0<a \in F$ such that $\mathcal{D}_{x}(f) \subset \mathcal{K}$ for a suitable element $0<b \in F$. Thereupon we are led to the contradiction of the supposition $S_{1}, S_{2} \subset \mathcal{W}_{1}^{(i)} \backslash \partial \mathcal{D}_{0}\left(R_{1}\right)$, as in the proof of Lemma 6 . Thus we conclude that the set $I^{(i)}$ consists of a single element. Denote $I^{(i)}=\{\rho(i)\}$ for 
a suitable bijective mapping $\rho: I_{1} \rightarrow I$.

Since $\bigcup_{i \in I_{1}} \mathcal{W}_{1}^{(i)} \cap \mathcal{D}_{0}\left(R_{2}\right)=\bigcup_{j \in J_{1}} \mathcal{W}_{2}^{(j)}$, we have that for every $i \in I_{1}$, and for an appropriate nonempty subset of indices $J_{1}^{(i)} \subset J_{1}, \mathcal{W}_{1}^{(i)} \subset \mathcal{D}_{0}\left(R_{2}\right)=$ $\bigcup_{j \in J_{1}^{(i)}} \mathcal{W}_{2}^{(j)}$, and moreover $\left\{J_{1}^{(i)}\right\}_{i \in I_{1}}$ forms a partition of the set $J_{1}$. On the other hand, we have proved above that the cardinalities of the sets $I$ and $I_{1}$ coincide, as do the cardinalities of the sets $J$ and $J_{1}$. Furthermore, the property of the bijective correspondence $\sigma$ implies the coincidence of the cardinalities of the sets $I$ and $J$. Because of that, each set $J_{1}^{(i)}$ consists of a single element. Let $J_{1}^{(i)}=\{\pi(i)\}$ for a suitable bijective correspondence $\pi: I_{1} \rightarrow J_{1}$. Thus, $\mathcal{W}_{1}^{(i)} \supset \mathcal{W}_{2}^{(\pi(i))}$ and we get the required diagram (8).

Now let $W$ be a connected component of the set $V \subset \tilde{F}^{n+2}$ (see (4)). We will prove that the intersection $W \cap \mathcal{D}_{0}\left(R_{3}\right)$ is connected for any $\tilde{F} \ni R_{3}>R$. This would imply the lemma. Assume the contrary and let $W^{(1)}$ and $W^{(2)}$ be the different connected components of the intersection $W \cap \mathcal{D}_{0}\left(R_{3}\right) \neq \emptyset$. By virtue of Lemma 7 there exists a unique connected component $\mathcal{W}$ of the set $\mathcal{V}$ which has a nonempty intersection with $W$; moreover $\mathcal{W} \supset W$.

First we suppose that the intersection $\mathcal{W} \cap \mathcal{D}_{0}\left(R_{3}\right)$ contains at least two connected components $\mathcal{W}^{(1)}, \mathcal{W}^{(2)}$ such that $\mathcal{W}^{(1)} \cap W^{(1)} \neq \emptyset$ and $\mathcal{W}^{(2)} \cap W^{(2)} \neq$ $\emptyset$. Let us prove the existence of an element $R_{4} \in \tilde{F}$ such that $R_{4}>R_{3}$ and such that for an $\tilde{F} \ni R_{5} \geq R_{4}$ the intersection $W \cap \mathcal{D}_{0}\left(R_{5}\right) \neq \emptyset$ is connected.

Indeed, there exists an element $R_{6} \in \tilde{F}$ such that for all $R_{5} \geq R_{6}$ the number of connected components of the intersection $W \cap \mathcal{D}_{0}\left(R_{5}\right)$ is the same, i.e., does not depend on $R_{5}$ (since the statement that $W \cap \mathcal{D}_{0}\left(R_{5}\right)$ has a given number of connected components can be expressed in terms of a certain formula of the theory of real closed fields with coefficients in the field $\tilde{F}$ ). Consider the semialgebraic set $U \subset \tilde{F}^{n+2}$, consisting of all points $u \in W$ in which the function $\left(X_{0}, \ldots, X_{n+1}\right) \rightarrow X_{0}^{2}+\cdots+X_{n+1}^{2}$ reaches a local minimum on $W$. Then on every connected component of the set $U$ the square of the norm has a constant value (see the beginning of the proof of this lemma). Choose $R_{4}^{2}$ to be larger than $R_{6}^{2}$ and $R^{2}$, and also larger than the values of the square of the norm on all connected components of the set $U$. We shall show that $R_{4}$ is the desired value. Assume the contrary. Then for some $\tilde{F} \ni R_{5} \geq R_{4}$ the intersection $W \cap \mathcal{D}_{0}\left(R_{5}\right)$ is disconnected. Let the points $y_{1}, y_{2} \in W \cap \mathcal{D}_{0}\left(R_{5}\right)$ be situated in the different connected components. Since $W$ is connected, there exists a closed bounded connected semialgebraic curve $C \subset W$ containing $y_{1}$ and $y_{2}$. Then $C \subset \mathcal{D}_{0}\left(R_{7}\right)$ for an appropriate $R_{5} \leq R_{7} \in F$. Since the numbers of connected components of the sets $W \cap \mathcal{D}_{0}\left(R_{5}\right)$ and $W \cap \mathcal{D}_{0}\left(R_{7}\right)$ coincide, there exists a connected component $W_{0}$ of the set $W \cap \mathcal{D}_{0}\left(R_{7}\right)$ such that $W_{0} \cap \mathcal{D}_{0}\left(R_{5}\right)=\emptyset$. 
Thus there exists a point $w_{0} \in W_{0}$ at which the square of the norm function reaches its minimum on $W_{0}$. Hence $w_{0} \in U$ and we obtain a contradiction with the choice of $R_{4}$. Thus, we have shown that $R_{4}$ has the desired property.

The above reasoning, with the aid of Proposition 3 (see the Introduction), allows us in fact to prove Lemma 8 with a somewhat worse bound on $R$. This remark will not be used in what follows.

A semialgebraic set $W_{4}=W \cap \mathcal{D}_{0}\left(R_{4}\right)$ can be determined by a formula of the theory of the real closed fields with coefficients in the field $\tilde{F}$. Then the set $W_{4}^{\left(\varepsilon_{1}, \varepsilon_{2}\right)} \subset F_{2}^{n+2}$, determined by the same formula, is also connected and $W_{4}^{\left(\varepsilon_{1}, \varepsilon_{2}\right)} \subset \mathcal{D}_{0}\left(R_{4}\right)$ because of the transfer principle. Furthermore, $W_{4}^{\left(\varepsilon_{1}, \varepsilon_{2}\right)} \subset \mathcal{V}$, the set $\mathcal{W}$ is connected and $\mathcal{W} \supset W$, and therefore $W_{4}^{\left(\varepsilon_{1}, \varepsilon_{2}\right)} \subset \mathcal{W}$. On the other hand, let $\mathcal{W} \cap \mathcal{D}_{0}\left(R_{4}\right)=\bigcup_{i} \mathcal{W}_{4}^{(i)}$ be the representation as the union of connected components. According to the results proved above (see the diagram (8)), there exists a bijective correspondence between these connected components and the connected components of the set $\mathcal{W} \cap \mathcal{D}_{0}\left(R_{3}\right)$. Let the connected components $\mathcal{W}_{4}^{(1)}$ and $\mathcal{W}_{4}^{(2)}$ correspond to the components $\mathcal{W}^{(1)}$ and $\mathcal{W}^{(2)}$ (see above). In particular $\mathcal{W}_{4}^{(1)} \supset \mathcal{W}^{(1)}$ and $\mathcal{W}_{4}^{(2)} \supset \mathcal{W}^{(2)}$. On the other hand, the connected set $W_{4}^{\left(\varepsilon_{1}, \varepsilon_{2}\right)} \subset \mathcal{W} \cap \mathcal{D}_{0}\left(R_{4}\right)$ satisfies $W_{4}^{\left(\varepsilon_{1}, \varepsilon_{2}\right)} \cap \mathcal{W} \neq \emptyset$ and $W_{4}^{\left(\varepsilon_{1}, \varepsilon_{2}\right)} \cap \mathcal{W}^{(2)} \neq \emptyset$, since $W^{(1)}, W^{(2)} \subset W_{4} \subset W_{4}^{\left(\varepsilon_{1}, \varepsilon_{2}\right)}$. Thus, we obtain a contradiction with the supposed existence of two connected components $\mathcal{W}^{(1)}, \mathcal{W}^{(2)}$ of the set $\mathcal{W} \cap \mathcal{D}_{0}\left(R_{3}\right)$ such that $\mathcal{W}^{(1)} \cap W^{(1)} \neq \emptyset$ and $\mathcal{W}^{(2)} \cap W^{(2)} \neq \emptyset$.

Finally let us suppose that there is a unique connected component $\mathcal{W}^{(3)}$ of the set $\mathcal{W} \cap \mathcal{D}_{0}\left(R_{3}\right)$ for which $\mathcal{W}^{(3)} \supset W \cap \mathcal{D}_{0}\left(R_{3}\right)$. Observe that $\mathcal{V} \cap \mathcal{D}_{0}\left(R_{3}\right) \supset$ $s t_{1}\left(\mathcal{V} \subset \mathcal{D}_{0}\left(R_{3}\right)\right)=V \cap \mathcal{D}_{0}\left(R_{3}\right)$ (see (4), (5)). Lemma 1 from Grigor'ev \& Vorobjov (1988) implies that for $\mathcal{W}^{(3)}$ (which is also a connected component of the set $\mathcal{V} \cap \mathcal{D}_{0}\left(R_{3}\right)$ ) there exists a unique connected component $W^{(3)}$ of the set $V \cap \mathcal{D}_{0}\left(R_{3}\right)$ such that $s t_{1}\left(\mathcal{W}^{(3)}\right) \subset W^{(3)}$. On the other hand, $s t_{1}\left(\mathcal{W}^{(3)}\right) \supset$ $\left(W \cap \mathcal{D}_{0}\left(R_{3}\right)\right) \supset\left(W^{(1)} \cup W^{(2)}\right)$. The contradiction thus obtained proves the connectedness of the set $W \cap \mathcal{D}_{0}\left(R_{3}\right)$.

Now we return to system (4) (see the beginning of the present section). Take $R$ from Lemma 8 and consider a semialgebraic set $V_{2}=V \cap \mathcal{D}_{0}(R) \subset \tilde{F}^{n+2}$, determined by the following system of inequalities: $f_{0} \equiv R^{2}-\left(X_{0}^{2}+\cdots+X_{n+1}^{2}\right) \geq$ $0, f_{1} \geq 0, \ldots, f_{k+4} \geq 0$. Also consider a set $\mathcal{V}_{2} \subset F_{2}^{n+2}$, determined by the system $f_{0}+\varepsilon_{1}>0, f_{1}+\varepsilon_{1}>0, \ldots, f_{k+4}+\varepsilon_{1}>0$ (see (5)), and a polynomial $g_{1}=\left(f_{0}+\varepsilon_{1}\right)\left(f_{1}+\varepsilon_{1}\right) \cdots\left(f_{k+4}+\varepsilon_{1}\right)+\varepsilon_{2}$ (see Lemma 3$)$. According to Lemma 6 there is a bijective correspondence between the connected components of the hypersurface $\left\{g_{1}=0\right\} \subset F_{2}^{n+2}$ which lies in $\mathcal{V}_{2}$ and the connected components of the set $\mathcal{V}_{2}\left(\right.$ clearly, $\left.\mathcal{V}_{2} \subset \mathcal{D}_{0}(R+1)\right)$. Lemma 7 implies the bijective correspon- 
dence between the connected components of the sets $\mathcal{V}_{2}$ and $V_{2}$ respectively. Finally, invoking Lemma 8, one gets the bijective correspondence between the connected components of the set $V_{2}$ and the connected components of the set $V$, and by the same token the connected components of the set $V_{0}$ (see (2)). In particular, the number of connected components of the set $V_{0}$ coincides with the number of connected components of the hypersurface $\left\{g_{1}=0\right\} \cap \mathcal{V}_{2}$, i.e., with the number of connected components of the hypersurface $\left\{g_{1}=0\right\}$ on which (everywhere, see Lemma 3 ) the polynomials $f_{0}+\varepsilon_{1}, f_{1}+\varepsilon_{1}, \ldots, f_{k+4}+\varepsilon_{1}$ are positive.

Below in Section 3 we will deal with the equation $g_{1}=0$. Recall that a semialgebraic set $\left\{g_{1}=0\right\}$ is a nonsingular hypersurface, since $\varepsilon_{2}$ is not a critical value of the polynomial $\left(f_{0}+\varepsilon_{1}\right)\left(f_{1}+\varepsilon_{1}\right) \cdots\left(f_{k+n}+\varepsilon_{1}\right) \in F_{1}\left[X_{0}, \ldots, X_{n+1}\right]$. Thus, the proof of the first statement of the theorem (see the Introduction) is reduced to considering a nonsingular bounded hypersurface $\left\{g_{1}=0\right\} \cap \mathcal{V}_{2}$.

In the remainder of this section we will also reduce the proof of the second statement of the theorem to considering the case of a nonsingular bounded hypersurface. Let $x=\left(x_{1}, \ldots, x_{n}\right) \in V_{0}$ and $y=\left(y_{1}, \ldots, y_{n}\right) \in V_{0}$. The algorithm has to test whether they belong to the same connected component of the set $V_{0}$. The latter condition is true if the points $x^{\prime}=\left(1 /\left(f_{1} \cdots f_{k_{1}}\right)(x)\right.$, $\left.x_{1}, \ldots, x_{n}, 0\right)=\left(x_{0}^{\prime}, x_{1}^{\prime}, \ldots, x_{n+1}^{\prime}\right)$ and $y^{\prime}=\left(1 /\left(f_{1} \cdots f_{k_{1}}\right)(y), y_{1}, \ldots, y_{n}, 0\right)$, $\left(y_{0}^{\prime}, y_{1}^{\prime}, \ldots, y_{n+1}^{\prime}\right)$ in $V \subset \tilde{F}^{n+2}$ are situated in the same connected component of the set $V$. Take $R^{\prime} \geq R$ (see Lemma 8) such that $R^{\prime} \in F$ is of the same kind as in Lemma 8, i.e., $R^{\prime}$ is an integer when $m=0$ or $R^{\prime}=\delta_{m}^{-s}$ when $m>0$, and the points $x^{\prime}, y^{\prime} \in \mathcal{D}_{0}\left(R^{\prime}\right)$. Introduce the polynomials $f_{0}^{\prime}=\left(R^{\prime}\right)^{2}-\left(x_{0}^{2}+\cdots+X_{n+1}^{2}\right)$ and $g_{1}^{\prime}=\left(f_{0}^{\prime}+\varepsilon_{1}\right)\left(f_{1}+\varepsilon_{1}\right) \cdots\left(f_{k+4}+\varepsilon_{1}\right)-\varepsilon_{2}$ (see above). By virtue of Lemma 8 the points $x^{\prime}, y^{\prime}$ lie in the same connected component of the set $V$ if $x^{\prime}, y^{\prime}$ lie in the same connected component of the set $V^{\prime}=V \cap \mathcal{D}_{0}\left(R^{\prime}\right)=\left\{f_{0}^{\prime} \geq 0 \wedge f_{1} \geq 0 \wedge \cdots \wedge f_{k+4} \geq 0\right\} \subset \tilde{F}^{n+1}$.

Let $x^{(0)}$ (respectively, $y^{(0)}$ ) be a point on the hypersurface $\left\{g_{1}^{\prime}=0\right\}$ nearest to the point $x^{\prime}$ (respectively, $y^{\prime}$ ). Consider a connected component $W^{\prime}$ of the set $V^{\prime}$ which contains $x^{\prime}$. Because of Lemma 7 there exists a unique connected component $\mathcal{W}^{\prime}$ of the set $\mathcal{V}^{\prime}=\left\{f_{0}^{\prime}+\varepsilon_{1}>0 \wedge f_{1}+\varepsilon_{2}>0 \wedge \cdots \wedge f_{k+4}+\varepsilon_{1}>\right.$ $0\} \subset F_{2}^{n+2}$ such that $\mathcal{W}^{\prime} \supset W^{\prime}$. Because of Lemma 6 there exists a unique connected component $S^{\prime}$ of the hypersurface $\left\{g_{1}^{\prime}=0\right\}$ for which $S^{\prime} \subset \mathcal{W}^{\prime}$.

We claim that $x^{(0)} \in S^{\prime}$. Assume the contrary. Then $x^{(0)}$ belongs to some connected component of the hypersurface $\left\{g_{1}^{\prime}=0\right\}$ which differs from $S^{\prime}$. Therefore, Lemma 6 implies that $x^{(0)} \notin \mathcal{W}^{\prime}$. In the closed segment $\left[x^{\prime}, x^{(0)}\right] \subset F_{2}^{n+2}$ of a line there exists the maximal semi-open segment $\left[x^{\prime}, x^{(1)}\right)$ situated in $\mathcal{W}^{\prime}$. It follows that $x^{(1)} \in \partial \mathcal{W}^{\prime}$. Note that $g_{1}^{\prime}\left(x^{\prime}\right) \geq \varepsilon_{1}^{k+5}-\varepsilon_{2}>0$ 
and $g_{1}^{\prime}\left(x^{(1)}\right)=-\varepsilon_{2}<0$. Hence there exists a point $x^{(2)} \in\left(x^{\prime}, x^{(1)}\right)$ for which $g_{1}^{\prime}\left(x^{(2)}\right)=0$, and we get a contradiction with the choice of $x^{(0)}$. This proves the claim $x^{(0)} \in S^{1}$.

Thus, the points $x, y$ belong to the same connected component of the set $V_{0}$ if the points $x^{(0)}, y^{(0)}$ belong to the same connected component of the hypersurface $\left\{g_{1}^{\prime}=0\right\}$.

Let the point $x^{\prime}$ be given in the following way (see the Introduction). For each coordinate $x_{i}^{\prime}$ a certain polynomial $\psi_{i} \in \mathbb{Q}_{m}[Z]$ and a sequence $\mathcal{S}_{i}$ of the signs of the derivatives of $\psi_{i}$ of all orders are fixed such that $x_{i}^{\prime}$ is the unique element satisfying the conditions $\psi_{i}\left(x_{i}^{\prime}\right)=0$ and $\mathcal{S}_{i}$. Introduce new variables $Z_{0}, \ldots, Z_{n+1}$. To find a point $x^{(0)}$ the algorithm applies Proposition 2 (see the Introduction) to the following system of inequalities (see (7) at the beginning of the proof of Lemma 8):

$$
\begin{gathered}
g_{1}^{\prime}(X)=0 ; \quad \psi_{l}\left(Z_{l}\right)=0, \quad \mathcal{S}_{l}, \text { for } 0 \leq l \leq n+1, \\
\left(X_{j}-Z_{j}\right) \frac{\partial g_{1}^{\prime}}{\partial X_{i}}=\left(X_{i}-Z_{i}\right) \frac{\partial g_{1}^{\prime}}{\partial X_{j}}, \text { for } 0 \leq i<j \leq n+1,
\end{gathered}
$$

and yields a representative set of points for the semialgebraic set $U \subset F_{2}^{2 n+2}$ determined by the latter system. Denote by $\pi$ a projection $\pi\left(X_{0}, \ldots, X_{n+1}\right.$, $\left.Z_{0}, \ldots, Z_{n+1}\right)=\left(X_{0}, \ldots, X_{n+1}\right)$. Then the projection $\pi(U)$ contains all local extremums of the distance from the point $x^{\prime}$ on the hypersurface $\left\{g_{1}^{\prime}=0\right\}$. Observe that on every connected component of the set $\pi(U)$ the distance from the point $x^{\prime}$ has a constant value (see the beginning of the proof of Lemma 8 above). Therefore there is a point among the yielded representative family for which the distance from the point $x^{\prime}$ is minimal on the hypersurface $\left\{g_{1}^{\prime}=0\right\}$. Again using Proposition 2 the algorithm compares the distances from the point $x^{\prime}$ for all the representative points and takes a representative point with the minimal distance as $x^{(0)}$. In a similar way the algorithm produces a point $y^{(0)}$.

Thus, we have reduced the proof of the second statement of the theorem to the case of a nonsingular bounded hypersurface, namely to testing whether two points $x^{(0)}, y^{(0)}$ belong to the same connected component of the hypersurface $\left\{g_{1}^{\prime}=0\right\}$.

\section{Producing a skeleton for connected components of a nonsingular bounded hypersurface}

A semialgebraic curve $\Sigma$ is called a skeleton of a semialgebraic set $U$ if $\Sigma \subset U$ and for every connected component $U_{1}$ of the set $U$ an intersection 
$\Sigma \cap U_{1}$ is nonempty and connected. In other words, $\Sigma \cap U_{1}$ is a connected component of the curve $\Sigma$.

As input the algorithm is given a polynomial $h \in F\left[X_{1}, \ldots, X_{n}\right]$ which satisfies a $\left(D, D_{0}, \mathcal{M}\right)$-bound, where the field $F=\mathbf{Q}\left(\delta_{1}, \ldots, \delta_{m}\right)$. In addition, an element $\mathcal{R}^{2} \in F$ is given, where $0<\mathcal{R} \in \tilde{F}$, such that $\mathcal{R}^{2}$ also satisfies a $\left(D, D_{0}, \mathcal{M}\right)$-bound. We assume in the following that the intersection $\{h=0\} \cap \partial \mathcal{D}_{0}(\mathcal{R})=\emptyset$. Finally, let the points $u^{(1)}=\left(u_{1}^{(1)}, \ldots, u_{n}^{(1)}\right), \ldots, u^{(p)}=$ $\left(u_{1}^{(p)}, \ldots, u_{n}^{(p)}\right) \in\{h=0\} \cap \mathcal{D}_{0}(\mathcal{R})$ be given, each of them also satisfying $\left(D, D_{0}, \mathcal{M}\right)$-bounds. The purpose of the present section is to produce a skeleton for the hypersurface $\{h=0\} \cap \mathcal{D}_{0}(\mathcal{R})$ which is required to pass through the points $u^{(1)}, \ldots, u^{(p)}$. The procedure will also produce the connected components of the skeleton.

To prove the first statement of Theorem 1 (see the Introduction) this procedure will be applied to the situation in which $g_{1}$ plays the role of the polynomial $h$ and $R^{2}+\varepsilon_{1}$ plays the role of $\mathcal{R}^{2}$ (see Section 2), and the number $p$ of the points is 0 . To prove the second statement of Theorem $1, g_{1}^{\prime}$ plays the role of $h$ and $\left(R^{\prime}\right)^{2}+\varepsilon_{1}$ plays the role of $\mathcal{R}^{2}$ and the points $u^{(1)}=x^{(0)}, u^{(2)}=y^{(0)}$ (see the very end of Section 2). Furthermore, in order to count the number of connected components and thereby to complete the proof of the first statement of the theorem, the algorithm selects only those connected components of the skeleton which lie inside the semialgebraic set $\left\{f_{1}+\varepsilon_{1}>0 \wedge \cdots \wedge f_{k+4}+\varepsilon_{1}>0\right\}$ (see (5) and Lemmas 3 and 6 in Section 2). For this it suffices to test the fulfilment of the latter inequalities for an arbitrary point of a connected component of the skeleton (such points will be produced by the algorithm as it proceeds).

The algorithm will work by recursion on some parameter $1 \leq l \leq n$. In the following we need to consider a more general situation than in Section 2. Namely, we consider for some $1 \leq l \leq n$ a variety

$$
\left\{h^{\left(\eta_{1}, \ldots, \eta_{n-l-1}\right)}\left(\eta_{1}, \ldots, \eta_{n-l}, Y_{1}, \ldots, Y_{l}\right)=0\right\} \subset \tilde{F}^{l}
$$

(in the space with the coordinates $Y_{1}, \ldots, Y_{l}$-from here on $\left(\eta_{1}, \ldots, \eta_{n-l-1}\right)$ is regarded as an upper multi-index) which has at most one singular point

$$
\sigma=\sigma^{\left(\eta_{1}, \ldots, \eta_{n-l}\right)}=\left(\sigma_{1}, \ldots, \sigma_{l}\right) \in\left\{h^{\left(\eta_{1}, \ldots, \eta_{n-l-1}\right)}\left(\eta_{1}, \ldots, \eta_{n-l}, Y_{1}, \ldots, Y_{l}\right)=0\right\},
$$

i.e., the point satisfying the following system of equations:

$$
\frac{\partial h^{\left(\eta_{1}, \ldots, \eta_{n-l-1}\right)}}{\partial Y_{1}}\left(\eta_{1}, \ldots, \eta_{n-l}, \sigma\right)=\cdots=\frac{\partial h^{\left(\eta_{1}, \ldots, \eta_{n-l-1}\right)}}{\partial Y_{l}}\left(\eta_{1}, \ldots, \eta_{n-l}, \sigma\right)=0
$$

We suppose here that the polynomial $h^{\left(\eta_{1}, \ldots, \eta_{n-l-1}\right)} \in F\left[Z_{1}, \ldots, Z_{n-l}\right]\left[Y_{1}, \ldots, Y_{l}\right]$ is already produced by recursion on $n-l$. In addition, we suppose that by the 
recursion on $1 \leq i \leq n-l$, polynomials $\varphi^{\left(\eta_{i}\right)}\left(Z_{i}\right) \in F\left[Z_{i}\right]$ are already produced such that $\varphi^{\left(\eta_{i}\right)}$ is irreducible over the field $F$ and it is monic, i.e., $l c_{z_{i}}\left(\varphi^{\left(\eta_{i}\right)}\right)=1$. Furthermore, for each $1 \leq i \leq n-l$, a sequence $\mathcal{S}^{\left(\eta_{i}\right)}$ of signs of the derivatives $\frac{d^{j} \varphi\left(\eta_{i}\right)}{d Z^{j}}$ for all $1 \leq j \leq \operatorname{deg}_{z_{i}}\left(\varphi^{\left(\eta_{i}\right)}\right)$ is already given. Recall (see Thom's lemma in the Introduction) that $\mathcal{S}^{\left(\eta_{i}\right)}$ defines the unique root $\eta_{i}$ of the polynomial $\varphi^{\left(\eta_{i}\right)}$ (provided that $\mathcal{S}^{\left(\eta_{i}\right)}$ is consistent). In addition, we suppose that a nonsingular linear transformation $\mathcal{M}^{\left(\eta_{1}, \ldots, \eta_{n-l-1}\right)}$ (defined by a matrix with the entries from Q) of $n$-dimensional space with the coordinates $X_{1}, \ldots, X_{n}$ into $n$-dimensional space with the coordinates $Z_{1}, \ldots, Z_{n-l}, Y_{1}, \ldots, Y_{l}$ is obtained such that

$$
h^{\left(\eta_{1}, \ldots, \eta_{n-l-1}\right)}\left(\mathcal{M}^{\left(\eta_{1}, \ldots, \eta_{n-l}\right)}\left(X_{1}, \ldots, X_{n}\right)\right)=h\left(X_{1}, \ldots, X_{n}\right) .
$$

Finally, let the points

$$
\begin{aligned}
u^{(1)}\left(\eta_{1}, \ldots, \eta_{n-l}\right) & =\left(\eta_{1}, \ldots, \eta_{n-l}, u_{1}^{(1)}, \ldots, u_{l}^{(1)}\right)^{T}, \ldots, u^{(q)}\left(\eta_{1}, \ldots, \eta_{n-l}\right) \\
& =\left(\eta_{1}, \ldots, \eta_{n-l}, u_{1}^{(q)}, \ldots, u_{l}^{(q)}\right)^{T} \in\left\{h^{\left(\eta_{1}, \ldots, \eta_{n-l-1}\right)}=0\right\} \\
& \cap \mathcal{M}^{\left(\eta_{1}, \ldots, \eta_{n-l-1}\right)} \mathcal{D}_{0}(\mathcal{R})
\end{aligned}
$$

be given, where each coordinate $u_{j_{2}}^{\left(j_{1}\right)} \in \tilde{F}$ is determined by its minimal polynomial $\varphi_{j_{1}, j_{2}}^{\left(\eta_{1}, \ldots, \eta_{n-l}\right)}$ and by a sequence $\mathcal{S}_{j_{1}, j_{2}}^{\left(\eta_{1}, \ldots, \eta_{n-l}\right)}$ of the signs of the derivatives of all orders of this polynomial. In a similar way $\sigma_{j_{2}}\left(1 \leq j_{2} \leq l\right)$ is determined by its minimal polynomial $\varphi_{\sigma_{j_{2}}}^{\left(\eta_{1}, \ldots, \eta_{n-l}\right)}$ and by a sequence $\mathcal{S}_{\sigma_{j_{2}}}^{\left(\eta_{1}, \ldots, \eta_{n-l}\right)}$ of signs of all its derivatives. We assume for uniformity that $\left(\eta_{1}, \ldots, \eta_{n-l}, \sigma\right)$ is contained among the points $u^{(1)}\left(\eta_{1}, \ldots, \eta_{n-l}\right), \ldots, u^{(q)}\left(\eta_{1}, \ldots, \eta_{n-l}\right)$.

Observe that the polynomials $\varphi^{\left(\eta_{i}\right)}$ and the sequences $\mathcal{S}^{\left(\eta_{i}\right)}$ for $1 \leq i \leq n-l$ fix the values of the coordinates $Z_{1}=\eta_{1}, \ldots, Z_{n-l}=\eta_{n-l}$; thus one can consider $h^{\left(\eta_{1}, \ldots, \eta_{n-l-1}\right)}$ as a polynomial in the variables $Y_{1}, \ldots, Y_{l}$.

The algorithm will produce, by recursion on $1 \leq l \leq n$, a skeleton for the union of all such connected components of the variety $\left\{h^{\left(\eta_{1}, \ldots, \eta_{n-l-1}\right)}\left(\eta_{1}, \ldots, \eta_{n-l}\right.\right.$, $\left.\left.Y_{1}, \ldots, Y_{l}\right)=0\right\} \subset\left(\tilde{F}^{n} \cap\left\{Z_{1}=\eta_{1} \wedge \cdots \wedge Z_{n-l}=\eta_{n-l}\right\}\right) \simeq \tilde{F}^{l}$ which lie in the set $\mathcal{M}^{\left(\eta_{1}, \ldots, \eta_{n-l-1}\right)} \mathcal{D}_{0}(\mathcal{R})$, and the skeleton contains the points $u^{(1)}\left(\eta_{1}, \ldots, \eta_{n-l}\right)$, $\ldots, u^{(q)}\left(\eta_{1}, \ldots, \eta_{n-l}\right)$. The recursive hypothesis claims that the algorithm can produce a skeleton for smaller values of $l$.

When $n=l$ the polynomials $\varphi$ with the suitable indices determine the coordinates of the points $u^{(1)}, \ldots, u^{(p)}$. The polynomial $h^{\left(\eta_{1}, \ldots, \eta_{n-l}\right)}=h$ was specified at the beginning of the section. For $l=1$ (the base of the recursion) the produced polynomials $\varphi^{\left(\eta_{i}\right)}$ and the sequences $\varphi^{\left(\eta_{i}\right)}, 1 \leq i \leq n-1$ determine uniquely the point $\left(\eta_{1}, \ldots, \eta_{n-1}\right)$. After that, from the roots $\eta_{n}$ of the polynomial $h^{\left(\eta_{1}, \ldots, \eta_{n-2}\right)}$ the algorithm selects (see below) those for which the inclusions $\left(\eta_{1}, \ldots, \eta_{n-1}, \eta_{n}\right) \in \mathcal{M}^{\left(\eta_{1}, \ldots, \eta_{n-2}\right)} \mathcal{D}_{0}(\mathcal{R})$ are true; they constitute a skeleton 
of a null-dimensional variety $\left\{h^{\left(\eta_{1}, \ldots, \eta_{n-2}\right)}\left(\eta_{1}, \ldots, \eta_{n-1}, Y_{1}\right)=0\right\} \cap \mathcal{M}^{\left(\eta_{1}, \ldots, \eta_{n-2}\right)}$, $\mathcal{D}_{0}(\mathcal{R}) \subset \tilde{F}^{n} \cap\left\{Z_{1}=\eta_{1} \wedge \cdots \wedge Z_{n-1}=\eta_{n-1}\right\}$.

Fix for some time the value $l$ and let for brevity

$$
H\left(Y_{1}, \ldots, Y_{l}\right)=h^{\left(\eta_{1}, \ldots, \eta_{n-l-1}\right)}\left(\eta_{1}, \ldots, \eta_{n-l}, Y_{1}, \ldots, Y_{l}\right) \in \tilde{F}\left[Y_{1}, \ldots, Y_{l}\right] .
$$

We also introduce the notation $a \approx b$ for two linearly dependent vectors and the notation $\operatorname{grad}_{x}(H)=\left(\frac{\partial H}{\partial Y_{1}}, \ldots, \frac{\partial H}{\partial Y_{l}}\right)(x)$ for the gradient of the polynomial $H$ at a point $x$.

Our next purpose is to find a direction in the space $\tilde{F}^{l}$, i.e., a vector $0 \neq t=$ $\left(t_{1}, \ldots, t_{l}\right) \in \mathbb{Z}^{l}$ such that for any two points $x, y \in\{H=0\}$ with $\operatorname{grad}_{x}(H) \approx$ $\left(t_{1}, \ldots, t_{l}\right) \approx \operatorname{grad}_{y}(H)$, the inner products $(x, t) \neq(y, t)$. In other words the points $x$ and $y$ are not both situated in the same hyperplane which is orthogonal to the vector $t$. In particular, taking into account that $\operatorname{grad}_{\sigma}(H)=0 \approx t$, the hyperplane orthogonal to $t$ and containing $\sigma$ does not contain any other point from $\{H=0\} \subset \tilde{F}$ with gradient collinear to $t$.

Consider a semialgebraic set $\mathcal{U}=\left\{(x, y)=\left(x_{1}, \ldots, x_{l}, y_{1}, y_{l}\right) \in \tilde{F}^{l} \times \tilde{F}^{l}\right.$ : $\left.H(x)=H(y)=0, \operatorname{grad}_{x}(H) \not \approx(y-x), \operatorname{grad}_{y}(H) \not \approx 0\right\} \subset \tilde{F}^{2 l}$, being a smooth manifold on its mapping $\rho_{1}: \mathcal{U} \rightarrow \tilde{F}^{l} \backslash\{0\}$ determined by the formula

$$
\rho_{1}(x, y)=\operatorname{grad}_{x}(H)-\frac{\left(\operatorname{grad}_{x}(H),(y-x)\right)}{\|y-x\|^{2}}(y-x),
$$

where the numerator of the fraction is the inner product of the vectors. Consider also a natural mapping $\rho_{2}: \tilde{F}^{l} \backslash\{0\} \rightarrow \mathrm{P}^{l-1}(\tilde{F})$ into the projective space over the field $\tilde{F}$ and a composition $\rho=\rho_{2} \rho_{1}: \mathcal{U} \rightarrow \mathrm{P}^{l-1}(\tilde{F})$.

One can check by a direct calculation that, for any pair of points $x, y$ such that $(x, y) \in \mathcal{U}$, the $\operatorname{gradients}_{\operatorname{grad}_{x}}(H) \approx \operatorname{grad}_{y}(H)$ are both distinct from zero and $\left((x-y), \operatorname{grad}_{x}(H)\right)=\left((x-y), \operatorname{grad}_{y}(H)\right)=0$; i.e., the point $(x, y)$ is a critical point of the mapping $\rho$. For this it suffices to show that the rank of the Jacobi matrix of the mapping $\rho$ at the point $(x, y)$ is less than l-1 (Hirsch 1976). To calculate the Jacobi matrix let us choose the local coordinates as follows. First, in the common hyperplane $\mathcal{T}$ tangent to the hypersurface $\{H=0\}$ at the points $x, y$ we choose some Cartesian coordinates $W_{1}, \ldots, W_{l-1}$ which are orthogonal and normalized with respect to the initial coordinates of the space $\tilde{F}^{l}$, and add a coordinate $W_{l}$ orthogonal to $\mathcal{T}$. Then, in a neighborhood of the point $(x, y)$, choose the $(2 l-2)$ local coordinates on $\mathcal{U}$ in such a way that the first $(l-1)$ coordinates $U_{1}, \ldots, U_{l-1}$ coincide with the coordinates $W_{1}, \ldots, W_{l-1}$ in a neighborhood of the point $x$ on $\mathcal{T}$ and in a similar way the last $(l-1)$ coordinates $V_{1}, \ldots, V_{l-1}$ coincide with $W_{1}, \ldots, W_{l-1}$ in a 
neighborhood of the point $y$. In the coordinate system $W_{1}, \ldots, W_{l-1}$, let the point $x$ have the coordinates $w_{1}, \ldots, w_{l-1}$ and the point $y$ have the coordinates $w_{1}^{\prime}, \ldots, w_{l-1}^{\prime}$. In the projective space $P^{l-1}(\tilde{F})=\left\{\left(T_{1}: \ldots: T_{l}\right)\right\}$ we choose an affine neighborhood $\left\{T_{1} \neq 0\right\}$ with the coordinates $T_{2} / T_{1}, \ldots, T_{l} / T_{1}$. In the chosen coordinates the $(l-1) \times 2(l-1)$ Jacobi matrix at the point $(x, y)$ has the form $(B O)$, where the $(l-1) \times(l-1)$ matrix

$$
B=\left(C-\frac{\left(w-w^{\prime}\right)\left(C^{T}\left(w-w^{\prime}\right)\right)^{T}}{\left\|w-w^{-1}\right\|^{2}}\right)\left(\frac{\partial H}{\partial W_{l}}(x)\right),
$$

where $w=\left(w_{1}, \ldots, w_{l-1}\right)$ and $w^{\prime}=\left(w_{1}^{\prime}, \ldots, w_{l-1}^{\prime}\right)$ are column vectors and $C$ is a matrix defined by

$$
C=\left(\frac{\partial^{2} H}{\partial U_{i} \partial U_{j}}(x)\right)_{i, j} .
$$

The matrix $B$ is singular since $\left(w-w^{\prime}\right)^{T} B=0$. This implies that the point $(x, y)$ is critical for the mapping $\rho$.

Consider now the semialgebraic set $\mathcal{U}_{\sigma}=\left\{x=\left(x_{1}, \ldots, x_{l}\right) \in \tilde{F}^{l}: H(x)=0\right.$, $\left.\operatorname{grad}_{x}(H) \not \approx(\sigma-x)\right\}$ (a smooth manifold), and a smooth mapping $\rho_{\sigma}=\left.\rho\right|_{y=\sigma}$, $\mathcal{U}_{\sigma} \rightarrow \mathrm{P}^{l-1}(\tilde{F})$ which is obtained from $\rho$ by substituting $\sigma$ for the point $y$. As above one can show that any point $x \in\{H=0\}$ with $x \neq \sigma$ such that $\left(\operatorname{grad}_{x}(H),(x-\sigma)\right)=0$, is a critical point for the mapping $\rho_{\sigma}$.

Observe that if a point $\rho_{2}\left(t_{1}, \ldots, t_{l}\right) \in \mathrm{P}^{l-1}(\tilde{F})$ does not belong to a semialgebraic set $\mathcal{K}$ which is a union of the images of the critical points of the mappings $\rho$ and $\rho_{\sigma}$, then the direction $\left(t_{1}, \ldots, t_{l}\right)$ satisfies the requirements formulated above. Sard's theorem (see Hirsch 1976) implies that $\operatorname{dim}_{\tilde{F}}(\mathcal{K})<l-1$ (here the transfer principle is also invoked).

Now we exhibit a finite family of vectors from $\mathbf{Z}^{l}$ such that not all of their images under the action of $\rho_{2}$ in $P^{l-1}(\tilde{F})$ lie in the set $\mathcal{K}$. Introduce a semialgebraic set $\mathcal{W}$ in the space $\tilde{F}^{n+2 l-1}$ with the coordinates $Z_{1}, \ldots, Z_{n-l}, X_{1}, \ldots, X_{l}$ $Y_{1}, \ldots, Y_{l}, T_{2}, \ldots, T_{l}$, determined by the following system of inequalities:

$$
\begin{gathered}
\sum_{1 \leq i \leq l}\left(X_{i}-Y_{i}\right)^{2} \neq 0 \\
\left(X_{1}-Y_{1}\right)+\sum_{2 \leq i \leq l} T_{i}\left(X_{i}-Y_{i}\right)=0 \\
h^{\left(\eta_{1}, \ldots, \eta_{n-l-1}\right)}\left(Z_{1}, \ldots, Z_{n-l}, X_{1}, \ldots, X_{l}\right) \\
=h^{\left(\eta_{1}, \ldots, \eta_{n-l-1}\right)}\left(Z_{1}, \ldots, Z_{n-l}, Y_{1}, \ldots, Y_{l}\right)=0
\end{gathered}
$$




$$
\begin{gathered}
\frac{\partial h^{\left(\eta_{1}, \ldots, \eta_{n-l-1}\right)}\left(Z_{1}, \ldots, Z_{n-l}, X_{1}, \ldots, X_{l}\right)}{\partial X_{i}} \\
=\frac{\partial h^{\left(\eta_{1}, \ldots, \eta_{n-l-1}\right)}\left(Z_{1}, \ldots, Z_{n-l}, X_{1}, \ldots, X_{l}\right)}{\partial X_{1}} T_{i}, \\
\frac{\partial h^{\left(\eta_{1}, \ldots, \eta_{n-l-1}\right)}\left(Z_{1}, \ldots, Z_{n-l}, Y_{1}, \ldots, Y_{l}\right)}{\partial Y_{i}} \\
=\frac{\partial h^{\left(\eta_{1}, \ldots, \eta_{n-l-1}\right)}\left(Z_{1}, \ldots, Z_{n-l}, Y_{1}, \ldots, Y_{l}\right)}{\partial Y_{1}} T_{i}, \text { for } 2 \leq i \leq l ; \\
\varphi^{\left(\eta_{j}\right)}\left(Z_{j}\right)=0, \mathcal{S}^{\left(\eta_{j}\right)}, \text { for } 1 \leq j \leq n-l .
\end{gathered}
$$

Introduce also a semialgebraic set $\mathcal{W}_{\sigma} \subset \tilde{F}^{n+2 l-1}$ in the space with the coordinates $Z_{1}, \ldots, Z_{n-l}, Z_{n-l+1}, \ldots, Z_{n}, X_{1}, \ldots, X_{l}, T_{2}, \ldots, T_{l}$ determined by the conjunction of system $(9 \mathrm{~b})$ and of the system

$$
\begin{gathered}
\sum_{1 \leq i \leq l}\left(X_{i}-Z_{n-l+i}\right)^{2} \neq 0, \\
\left(X_{1}-Z_{n-l+1}\right)+\sum_{2 \leq i \leq l} T_{i}\left(X_{i}-Z_{n-l+i}\right)=0, \\
h^{\left(\eta_{1}, \ldots, \eta_{n-l-1}\right)}\left(Z_{1}, \ldots, Z_{n-l}, X_{1}, \ldots, X_{l}\right)=0, \\
\frac{\partial h^{\left(\eta_{1}, \ldots, \eta_{n-l-1}\right)}\left(Z_{1}, \ldots, Z_{n-l}, X_{1}, \ldots, X_{l}\right)}{\partial X_{i}} \\
=\frac{\partial h^{\left(\eta_{1}, \ldots, \eta_{n-l-1}\right)}\left(Z_{1}, \ldots, Z_{n-l}, X_{1}, \ldots, X_{l}\right)}{\partial X_{1}} T_{i}, \text { for } 2 \leq i \leq l ; \\
\varphi_{\sigma_{j}}^{\left(\eta_{1}, \ldots, \eta_{n-l}\right)}\left(Z_{n-l+j}\right)=0, \mathcal{S}_{\sigma_{j}}^{\left(\eta_{1}, \ldots, \eta_{n-l}\right)}\left(Z_{n-l+j}\right), \text { for } 1 \leq j \leq l .
\end{gathered}
$$

Consider the projections $\pi: \tilde{F}^{n+2 l-1} \rightarrow \tilde{F}^{l-1}$ and $\pi_{1}: \tilde{F}^{n+2 l-1} \rightarrow \tilde{F}^{l-1}$ of the two spaces introduced above onto the coordinates $T_{2}, \ldots, T_{l}$. Note that if a direction $\left(1, t_{2}, \ldots, t_{l}\right) \in \tilde{F}^{l}$ satisfies the above formulated requirement (i.e., for any two points $x, y \in\{H=0\}$ such that $\operatorname{grad}_{x}(H) \approx\left(1, t_{2}, \ldots, t_{l}\right) \approx \operatorname{grad}_{y}(H)$ holds, $(x, t) \neq(y, t))$, then the vector $\left(t_{2}, \ldots, t_{l}\right)$ does not belong to the union of the projections $\pi(\mathcal{W}) \cup \pi_{1}\left(\mathcal{W}_{\sigma}\right)$. Furthermore $\operatorname{dim}_{\tilde{F}}\left(\pi(\mathcal{W}) \cup \pi_{1}\left(\mathcal{W}_{\sigma}\right)\right)<l-1$ since $\rho_{2}\left(\pi(\mathcal{W}) \cup \pi_{1}\left(\mathcal{W}_{\sigma}\right)\right) \subset \mathcal{K}$.

Next, the algorithm applies Proposition 3 to the family of polynomials occurring in the system (9a), (9b), and to the projection $\pi$. As a result the algorithm yields a family of polynomials $g_{0}^{(i)} \in F\left[T_{2}, \ldots, T_{l}\right]$ for $1 \leq i \leq N_{0}$ (the bounds on the degrees and on the lengths of coefficients of the polynomials 
$g_{0}^{(i)}$ and also on $N_{0}$ will be obtained later in Section 5). In a similar way the algorithm applies Proposition 3 to the family of polynomials from the system (9b), (9c) and to the projection $\pi_{1}$. As a result the algorithm yields a family of polynomials $g_{1}^{(i)} \in F\left[T_{2}, \ldots, T_{l}\right]$ for $1 \leq i \leq N_{1}$ (their bounds will be also obtained in Section 5). Proposition 3 implies the inclusion

$$
\pi(\mathcal{W}) \cup \pi_{1}\left(\mathcal{W}_{\sigma}\right) \subset\left\{g^{\prime} \equiv \prod_{1 \leq i \leq N_{0}} g_{0}^{(i)} \cdot \prod_{1 \leq i \leq N_{1}} g_{1}^{(i)}=0\right\}
$$

taking into account the inequality $\operatorname{dim}_{\tilde{F}}\left(\pi(\mathcal{W}) \cup \pi_{1}\left(\mathcal{W}_{\sigma}\right)\right)<l-1$ proven above.

Let $\operatorname{deg}_{T_{2}, \ldots, T_{l}}\left(g^{\prime}\right) \leq N^{\prime}$ (a bound on $N^{\prime}$ will be obtained in Section 5 ). Then at least one of the integer vectors $\left(t_{2}, \ldots, t_{l}\right)$ from the set $\left\{0,1, \ldots, N^{\prime}\right\}^{l-1}$ does not belong to the variety $\left\{g^{\prime}=0\right\}$.

Thus, the algorithm looks over all the vectors $\left(t_{2}, \ldots, t_{l}\right) \in\left\{0,1, \ldots, N^{\prime}\right\}^{l-1}$, for each of them substitutes $t_{2}, \ldots, t_{l}$ instead of $T_{2}, \ldots, T_{l}$ respectively, in both systems (9a), (9b) and (9b), (9c), and tests with the aid of Proposition 2 whether each of the systems is solvable in $\tilde{F}^{n+l}$. The vector $\left(t_{2}, \ldots, t_{l}\right)$ is the desired vector if both are unsolvable. We summarize what was proved above in the following lemma, using the notation introduced above.

LEMMA 9. At least one of the integer vectors of the form $\left(1, t_{2}, \ldots, t_{l}\right)$, where $0 \leq t_{i} \leq N^{\prime}$ and $2 \leq i \leq l$, satisfies the following requirement: any two distinct points $x, y \in\{H=0\}$ for which $\operatorname{grad}_{x}(H) \approx\left(1, t_{2}, \ldots, t_{l}\right) \approx \operatorname{grad}_{y}(H)$, do not both lie in a hyperplane which is orthogonal to the vector $\left(1, t_{2}, \ldots, t_{l}\right)$.

Let $\left(1, t_{2}, \ldots, t_{l}\right)$ be the vector found satisfying Lemma 9 . Introduce the $l \times l$ matrix

$$
\mathcal{B}=\left(\begin{array}{cccc}
1 & t_{2} & \ldots & t_{l} \\
& 1 & & 0 \\
& & \ddots & \\
0 & & & 1
\end{array}\right)
$$

and let $\bar{h}^{\left(\eta_{1}, \ldots, \eta_{n-l-1}\right)}=h^{\left(\eta_{1}, \ldots, \eta_{n-l-1}\right)}\left(Z_{1}, \ldots, Z_{n-l}, \quad \mathcal{B}^{-1}\left(\bar{Y}_{1}, \ldots, \bar{Y}_{l}\right)^{T}\right) \epsilon$ $F\left[Z_{1}, \ldots, Z_{n-l}, \bar{Y}_{1}, \ldots, \bar{Y}_{l}\right]$. Finally, a polynomial

$$
h^{\left(\eta_{1}, \ldots, \eta_{n-l}\right)}\left(Z_{1}, \ldots, Z_{n-l}, Z_{n-l+1}, Y_{1}, \ldots, Y_{l-1}\right)
$$

is obtained from $\bar{h}^{\left(\eta_{1}, \ldots, \eta_{n-l-1}\right)}$ by replacing $\bar{Y}_{1}, \ldots, \bar{Y}_{l}$ by $Z_{n-l+1}, Y_{1}, \ldots, Y_{l-1}$, respectively. Thus, one can introduce a linear transformation of $n$-dimensional 
space

$$
\mathcal{M}^{\left(\eta_{1}, \ldots, \eta_{n-l-1}, \eta_{n-l}\right)}=\left(\begin{array}{cccc}
1 & t_{2} & \ldots & t_{l} \\
& 1 & & 0 \\
& & \ddots & \\
0 & & & 1
\end{array}\right) \mathcal{M}^{\left(\eta_{1}, \ldots, \eta_{n-l-1}\right)}
$$

(see the above recursive hypothesis).

The system of inequalities

$$
\begin{gathered}
h^{\left(\eta_{1}, \ldots, \eta_{n-l}\right)}=\frac{\partial h^{\left(\eta_{1}, \ldots, \eta_{n-l}\right)}}{\partial Y_{1}}=\cdots=\frac{\partial h^{\left(\eta_{1}, \ldots, \eta_{n-l}\right)}}{\partial Y_{l-1}}=0, \\
\varphi^{\left(\eta_{i}\right)}\left(Z_{i}\right)=0, \quad \mathcal{S}^{\left(\eta_{i}\right)}, \text { for } 1 \leq i \leq n-l
\end{gathered}
$$

has a finite number of solutions in $\tilde{F}^{n}$. To prove this, consider a smooth variety $\mathcal{W}=\left\{h^{\left(\eta_{1}, \ldots, \eta_{n-l}\right)}=0 ; \varphi^{\left(\eta_{i}\right)}\left(Z_{i}\right)=0, \mathcal{S}^{\left(\eta_{i}\right)}, 1 \leq i \leq n-l\right\} \backslash\left(\eta_{1}, \ldots, \eta_{n-l}\right.$, $\left.\mathcal{B}\left(\sigma_{1}, \ldots, \sigma_{l}\right)^{T}\right) \subset \tilde{F}^{n}$ (taking into account that $\sigma$ is the unique singular point of the variety $\left.\left\{h^{\left(\eta_{1}, \ldots, \eta_{n-l-1}\right)}\left(\eta_{1}, \ldots, \eta_{n-l}, Y_{1}, \ldots, Y_{l}\right)=0\right\}\right)$ and its projection $\pi_{n-l+1}$ onto the coordinate $Z_{n-l+1}$. The set of critical points of the function $\pi_{n-l+1}$ on the variety $\mathcal{W}$ coincides with the set of solutions of system (10a), (10b). By virtue of Sard's theorem there are only a finite number of critical values of the function $\pi_{n-l+1}$. All the critical points together with the point $\left(\eta_{1}, \ldots, \eta_{n-l}, \mathcal{B}\left(\sigma_{1}, \ldots, \sigma_{l}\right)^{T}\right)$ have pairwise distinct values under the function $\pi_{n-l+1}$ because of the choice of the vector $\left(1, t_{2}, \ldots, t_{l}\right)$ (see Lemma 9 ). Hence, the number of the critical points is also finite.

In what follows the algorithm produces a finite set of options from $\tilde{F}$ for an element $\eta_{n-l+1}$. The algorithm considers all these options and branches according to them. The algorithm involves three procedures for producing the options for $\eta_{n-l+1}$, which we describe below.

With the help of Proposition 2 we find all the solutions in $\tilde{F}^{n}$ of the system (10a), (10b). The algorithm considers all these solutions. Fix one of these solutions $w=\left(\eta_{1}, \ldots, \eta_{n-l}, w_{n-l+1}, \ldots, w_{n}\right) \in \tilde{F}^{n}$. With the aid of Proposition 2 the algorithm tests whether the point $w$ belongs to the set $\mathcal{M}^{\left(\eta_{1}, \ldots, \eta_{n-l}\right)} \mathcal{D}_{0}(\mathcal{R})$. If not then the solution $w$ is discarded. Because of this we assume that $w \in$ $\mathcal{M}^{\left(\eta_{1}, \ldots, \eta_{n-l}\right)} \mathcal{D}_{0}(\mathcal{R})$. Relying on Proposition 2 the algorithm yields an irreducible polynomial $\varphi^{\left(\eta_{n-l+1}\right)} \in F\left[Z_{n-l+1}\right]$ for which $\varphi^{\left(\eta_{n-l+1}\right)}\left(w_{n-l+1}\right)=0$, and also a sequence $\mathcal{S}^{\left(\eta_{n-1+1}\right)}$ of signs of the derivatives of all the orders of the polynomial $\varphi^{\left(\eta_{n-l+1}\right)}$ such that $\mathcal{S}^{\left(\eta_{n-l+1}\right)}$ determines the root $w_{n-l+1}$ of $\varphi^{\left(\eta_{n-l+1}\right)}$. Thus, according to the first procedure, the algorithm sets an option $\eta_{n-l+1}=w_{n-l+1}$. Finally, we put $\sigma\left(\eta_{1}, \ldots, \eta_{n-l+1}\right)=w$. 
According to the second procedure the algorithm sets an element $\eta_{n-l+1}$ to be equal to $u_{1}^{(i)}+\sum_{2 \leq j \leq l} t_{j} u_{j}^{(i)}$ (for different $\left.1 \leq i \leq q\right)$; i.e., to the $(n+l-1)$-th coordinate of the vector

$$
\left(\begin{array}{llll}
1 & & & \mathbf{O} \\
& \ddots & & \\
0 & & 1 & \\
& & & \mathcal{B}
\end{array}\right)\left(u^{(i)}\left(\eta_{1}, \ldots, \eta_{n-l}\right)\right) .
$$

Denote by $F^{\left(\eta_{1}, \ldots, \eta_{n-l}, \eta_{n-l+1}\right)}$ a plane of the kind $\left\{Z_{1}=\eta_{1} \wedge \cdots \wedge Z_{n-l}=\right.$ $\left.\eta_{n-l} \wedge Z_{n-l+1}=\eta_{n-l+1}\right\} \subset \tilde{F}^{n}$, and by $F=\bigcup_{\eta_{n-l+1}} F^{\left(\eta_{1}, \ldots, \eta_{n-l}, \eta_{n-l+1}\right)}$ the union of such planes ranging over all the options of an element $\eta_{n-l+1}$ according to the first procedure. Introduce also a polynomial $\bar{H}=h^{\left(\eta_{1}, \ldots, \eta_{n-l}\right)}\left(\eta_{1}, \ldots, \eta_{n-l}\right.$, $\left.Z_{n-l+1}, Y_{1}, \ldots, Y_{l-1}\right) \in \tilde{F}\left[Z_{n-l+1}, Y_{1}, \ldots, Y_{l-1}\right]$, a polynomial $\bar{\Delta}^{=}$ $\sum_{1 \leq i \leq l-1}\left(\frac{\partial \bar{H}}{\partial Y_{i}}\right)^{2}$, and an integer $N_{2}=(2 D)^{l}$. The proof of the following lemma is similar to the proof of Lemma 5 in Grigor'ev \& Vorobjov (1988).

LEMma 10. There exist integers $0 \leq \lambda_{1}, \ldots, \lambda_{l-q} \leq N_{2}$ such that for every $0 \leq i \leq l-q$ and each component $W \subset \bar{F}^{l}$ (irreducible over the field $F$ ) of a variety determined by a system of equations

$$
\bar{H}=\left(\frac{\partial H}{\partial Y_{1}}\right)^{2}-\frac{\lambda_{1}}{N_{2} l} \bar{\Delta}=\cdots=\left(\frac{\partial \bar{H}}{\partial Y_{i}}\right)^{2}-\frac{\lambda_{i}}{N_{2} l} \bar{\Delta}=0
$$

for which the intersection $W \cap\left(\tilde{F}^{l} \backslash F\right) \neq \emptyset$ is nonempty, we have the equality $\operatorname{dim}_{F}(W)=l-i-1$.

Proof. The proof proceeds by induction on $i$. The base of $i=0$ is obvious since the equation $\bar{H}=0$ determines a hypersurface in $\bar{F}^{l}$. Suppose that for $i-1$ the statement of the lemma is ascertained. We shall prove it for $i \leq l-1$. Let $W_{1}, \ldots, W_{s} \subset \bar{F}^{l}$ be all the irreducible components of the variety determined by system $(11)_{i-1}$ such that $W_{j} \cap\left(\tilde{F}^{l} \backslash F\right) \neq \emptyset$ for $1 \leq j \leq s$. Then $\operatorname{dim}_{F}\left(W_{j}\right)=l-i$ by the inductive hypothesis. Because of Bezout's theorem (see, e.g., Shafarevich 1974) the number of components $s \leq(2 D)^{i}<N_{2}$. For any $1 \leq j \leq s$ there can exist at most one value of $x$ for which the polynomial $\left(\frac{\partial \bar{H}}{\partial Y_{i}}\right)^{2}-\frac{x}{N_{2} l} \bar{\Delta}$ vanishes everywhere on $W_{j}$, taking into account that $\bar{\Delta}$ does not vanish anywhere on $W_{j} \cap\left(\tilde{F}^{l} \backslash F\right)$. Therefore, there exists an integer $0 \leq \lambda_{i} \leq s \leq N_{2}$ such that the polynomial $\left(\frac{\partial \bar{H}}{\partial Y_{i}}\right)^{2}-\frac{\lambda_{i}}{N_{2} l} \bar{\Delta}$ does not vanish everywhere on each component $W_{j}, 1 \leq j \leq s$. 
The algorithm tests all possible $0 \leq \lambda_{1}, \ldots, \lambda_{l-2} \leq N_{2}$ and finds among them an $(l-2)$-tuple $\lambda_{1}, \ldots, \lambda_{l-2}$ satisfying the requirements from Lemma 10 in the following way. Introduce a polynomial

$$
\Delta=\sum_{1 \leq i \leq l-1}\left(\frac{\partial h^{\left(\eta_{1}, \ldots, \eta_{n-l}\right)}}{\partial Y_{i}}\right)^{2},
$$

and for any $(l-2)$-tuple $0 \leq \lambda_{1}, \ldots, \lambda_{l-2} \leq N_{2}$, consider a system of equations in the variables $Z_{1}, \ldots, Z_{n-l}, Z_{n-l+1}, Y_{1}, \ldots, Y_{l-1}$ (see $(11)_{l-2}$ ):

$$
\begin{aligned}
& h^{\left(\eta_{1}, \ldots, \eta_{n-l}\right)}=\left(\frac{\partial h^{\left(\eta_{1}, \ldots, \eta_{n-l}\right)}}{\partial Y_{1}}\right)-\frac{\lambda_{1}}{N_{2} l} \Delta \\
&=\cdots=\left(\frac{\partial h^{\left(\eta_{1}, \ldots, \eta_{n-l}\right)}}{\partial Y_{l-2}}\right)^{2}-\frac{\lambda_{n-2}}{N_{2} l} \Delta=0, \\
& \varphi^{\left(\eta_{i}\right)}\left(Z_{i}\right)=0, \text { for } 1 \leq i \leq n-l .
\end{aligned}
$$

Applying Proposition 1 (see the Introduction) the algorithm finds all the components, irreducible over the field $F$, of the variety determined by system (12). For each of these components $W^{(1)} \subset \bar{F}^{n}$ the algorithm produces a family of polynomials $g_{1}, \ldots, g_{\tau} \in F\left[Z_{1}, \ldots, Z_{n-l+1}, Y_{1}, \ldots, Y_{l-1}\right]$ such that $W^{(1)}$ coincides with the variety of all solutions of the system $g_{1}=\cdots=g_{\tau}=0$. Using Proposition 2 the algorithm tests the solvability in the space $\tilde{F}^{n}$ of the following quantifier-free formula:

$$
\begin{gathered}
g_{1}=\cdots=g_{\tau}=0 \\
T\left(\bigvee_{\eta_{n-l+1}}\left(\varphi^{\left(\eta_{n-l+1}\right)}\left(Z_{n-l+1}\right)=0 \wedge \mathcal{S}^{\left(\eta_{n-l+1}\right)}\left(Z_{n-l+1}\right)\right)\right) \\
\mathcal{S}^{\left(\eta_{i}\right)}, 1 \leq i \leq n-l
\end{gathered}
$$

where the disjunction ranges over all the options for an element $\eta_{n-l+1}$ according to the first procedure (see above). A tuple $\lambda_{1}, \ldots, \lambda_{l-2}$ satisfies the requirements from Lemma 10 if formula $(13 \mathrm{a}, \mathrm{b})$ is unsolvable for any irreducible component $W^{(1)}$ whose dimension $\operatorname{dim}_{F}\left(W^{(1)}\right)>1$. Lemma 10 implies the existence of a tuple with the latter property.

Thus, we assume in what follows that $\lambda_{1}, \ldots, \lambda_{l-2}$ satisfy Lemma 10. Denote by $\mathcal{C} \subset\left(\tilde{F}^{n} \cap\left\{Z_{1}=\eta_{1} \wedge \cdots \wedge Z_{n-l}=\eta_{n-l}\right\}\right) \backslash F$ a semialgebraic set determined by a conjunction of systems (12), (13b). 
LEMMA 11. (1) The dimension $\operatorname{dim}_{\tilde{F}}(C) \leq 1$;

(2) For every connected component $U$ of the variety $\{h=0\} \subset \tilde{F}^{n}$ such that $U \subset \mathcal{D}_{0}(\mathcal{R})$ (see the beginning of the section), and any element $\alpha \in \tilde{F}$ distinct from all options for $\eta_{n-l+1}$, if $U_{0}=\mathcal{M}^{\left(\eta_{1}, \ldots, \eta_{n-l}\right)} U \cap\left\{Z_{1}=\right.$ $\left.\eta_{1} \wedge \cdots \wedge Z_{n-l}=\eta_{n-l} \wedge Z_{n-l+1}=\alpha\right\} \neq \emptyset$ is nonempty then each connected component of the set $U_{0}$ has a nonempty intersection with $C$.

Proof. Part (1) was in fact ascertained above by taking into account that for any irreducible component $W^{(1)} \subset \bar{F}^{n}$ (see (12), (13a)) we have $\operatorname{dim}_{\bar{F}}\left(W^{(1)} \cap\right.$ $\left.\tilde{F}^{n}\right) \leq \operatorname{dim}_{\bar{F}}\left(W^{(1)}\right)$.

(2) Observe that $U_{0}$ is a bounded closed variety. Moreover, $U_{0}$ is a nonsingular hypersurface in the subspace $\left\{Z_{1}=\eta_{1} \wedge \cdots \wedge Z_{n-l}=\eta_{n-l} \wedge Z_{n-l+1}=\alpha\right\}$, since the gradient $\left(\frac{\partial \bar{H}}{\partial Y_{1}}, \ldots, \frac{\partial \bar{H}}{\partial Y_{l-1}}\right)$ does not vanish on $U_{0}$ by virtue of the choice of $\alpha$ (see system (10a), (10b)). Because of this there exists a point in $U_{0}$ such that the direction of the gradient is as required in system (12) (see Lemma 4 of Grigor'ev \& Vorobjov (1988)).

Our next goal is to find all local extremums of the coordinate function $Z_{n-1+1}$ on the connected components (note that they are either one-dimensional or null-dimensional) of the curves of the type $W^{(1)} \cap \tilde{F}^{n}$, where $W^{(1)}=\left\{g_{1}=\right.$ $\left.\cdots=g_{\tau}=0\right\} \subset \bar{F}^{n}$ is a one-dimensional irreducible component of the variety determined by system (12).

Consider a polynomial $G=\sum_{1 \leq i \leq \tau} g_{i}^{2}$. Introduce an element $\varepsilon>0$ which is infinitesimal with respect to the field $F$, and denote the corresponding standard part by st. Consider a nonsingular hypersurface $\mathcal{U}_{\varepsilon}=\{G=\varepsilon\} \subset(\tilde{F}(\varepsilon))^{n}$. By virtue of Lemma 3 from Grigor'ev \& Vorobjov (1988), for any point $x \in \tilde{F}^{n}$ such that $G(x)=0$ (i.e., $x \in W^{(1)} \cap \tilde{F}^{n}$ and in particular $x$ satisfies system (12)) there exists a point $y \in \mathcal{U}_{\varepsilon}$ for which $\operatorname{st}(y)=x$.

Consider a connected component $C_{1}$ of the curve $W^{(1)} \cap \tilde{F}^{n}$ such that $C_{1} \subset$ $\mathcal{M}^{\left(\eta_{1}, \ldots, \eta_{n-l}\right)} U \cap\left\{Z_{1}=\eta_{1} \wedge \cdots \wedge Z_{n-l}=\eta_{n-l}\right\}$ for a certain connected component $U \subset \mathcal{D}_{0}(\mathcal{R})$ of the variety $\{h=0\}$, and suppose that at some point $x=$ $\left(\eta_{1}, \ldots, \eta_{n-l}, Z_{n-l+1}, y_{1}, \ldots, y_{l-1}\right) \in C_{1}$ the function $Z_{n-l+1}$ reaches a local extremum on the set $C_{1}$ (let this be the maximum for definiteness). Take a point $y \in \mathcal{U}_{\varepsilon}$ for which $s t(y)=x$ (see above). Pick out $\beta_{1}, \beta_{2} \in \tilde{F}$ such that $\beta_{1}<z_{n-l+1}<\beta_{2}$ and the function $Z_{n-l+1}$ reaches a maximum at the point $x$ on the connected component $C_{1}^{(1)}$ of the set $C_{1} \cap\left\{\beta_{1} \leq Z_{n-l+1} \leq\right.$ $\left.\beta_{2}\right\}$ which contains the point $x$. The required $\beta_{1}, \beta_{2}$ exist since either $C_{1} C$ $\left\{Z_{n-l+1}=\alpha\right\}$ for some $\alpha$, or any intersection $C_{1} \cap\left\{Z_{n-l+1}=\alpha\right\}$ is either empty or finite, taking into account the irreducibility of $W^{(1)}$ and the theorem 
on the dimension of intersection (see, e.g., Shafarevich 1974). There is a unique connected component $\mathcal{U}_{\varepsilon}^{(1)}$ of the closed semialgebraic set $\mathcal{U}_{\varepsilon} \cap\left\{\beta_{1} \leq Z_{n-l+1} \leq\right.$ $\left.\beta_{2}\right\}$ such that $y \in \mathcal{U}_{\varepsilon}^{(1)}$. Then $s t\left(\mathcal{U}_{\varepsilon}^{(1)}\right) \subset W^{(1)} \cap \tilde{F}^{n}$ (the standard part st is definable everywhere on $\mathcal{U}_{\varepsilon}^{(1)}$ since

$$
\mathcal{U}_{\varepsilon} \cap \partial \mathcal{M}^{\left(\eta_{1}, \ldots, \eta_{n-l}\right)} \mathcal{D}_{0}(\mathcal{R})=\emptyset
$$

taking into account the property of $h$ at the beginning of the section, hence $\left.\mathcal{U}_{\varepsilon}^{(1)} \subset \mathcal{M}^{\left(\eta_{1}, \ldots, \eta_{n-l}\right)} \mathcal{D}_{0}(\mathcal{R})\right)$, and therefore we get the inclusions st $\left(\mathcal{U}_{\varepsilon}^{(1)}\right) \subset C_{1} \cap$ $\left\{\beta_{1} \leq Z_{n-l+1} \leq \beta_{2}\right\}$ and also st $\left(\mathcal{U}_{\varepsilon}^{(1)}\right) \subset C_{1}^{(1)}$ using Lemma 1 from Grigor'ev $\&$ Vorobjov (1988). Therefore, the function $Z_{n-l+1}$ reaches its maximum on the bounded closed algebraic set $\mathcal{U}_{\varepsilon}^{(1)}$ at a certain point $y^{(1)}=\left(z_{1}^{(1)}, \ldots, z_{n-l}^{(1)}\right.$, $\left.z_{n-l+1}^{(1)}, y_{1}^{(1)}, \ldots, y_{l-1}^{(1)}\right)$. Moreover $\operatorname{st}\left(z_{n-l+1}^{(1)}\right)=z_{n-l+1}$ because of the inclusion $s t\left(\mathcal{U}_{\varepsilon}^{(1)}\right) \subset C_{1}^{(1)}$. In particular, $Z_{n-l+1}$ reaches at the point $y^{(1)}$ a local maximum on the variety $\mathcal{U}_{\varepsilon}$. Hence, the gradient $0 \neq \operatorname{grad}_{y(1)}(G) \approx(\underbrace{0, \ldots, 0,1}_{n-l+1}, 0, \ldots, 0)$, since $\mathcal{U}_{\varepsilon}$ is a nonsingular hypersurface.

To find the local extremums the algorithm yields, with the aid of Proposition 2, a representative set of the variety $\mathcal{W}$ determined in the space $(\tilde{F}(\varepsilon))^{n}$ by the following system of equations:

$$
G-\varepsilon=\frac{\partial G}{\partial Z_{1}}=\cdots=\frac{\partial G}{\partial Z_{n-l}}=\frac{\partial G}{\partial Y_{1}}=\cdots=\frac{\partial G}{\partial Y_{l-1}}=0 .
$$

For each representative point $y^{\prime}=\left(z_{1}^{\prime}, \ldots, z_{n-l+1}^{\prime}, y_{1}^{\prime}, \ldots, y_{l-1}^{\prime}\right)$ produced, the algorithm constructs its standard part $\operatorname{st}\left(y^{\prime}\right) \in \tilde{F}^{n}$ (provided that it is definable, otherwise we do not consider $y^{\prime}$ in the following) using Lemma 8 from Grigor'ev \& Vorobjov (1988) and also Proposition 2.

We claim that among the constructed standard parts there is a point $x^{\prime} \in C_{1}$ at which the function $Z_{n-l+1}$ reaches a local maximum $z_{n-l+1}$ on $C_{1}$. Indeed, since the point $y^{(1)} \in \mathcal{W}$, there exists a representative point $y^{\prime \prime}=\left(z_{1}^{\prime \prime}, \ldots, z_{n-l}^{\prime \prime}\right.$, $\left.z_{n-l+1}^{\prime \prime}, y_{1}^{\prime \prime}, \ldots, y_{l-1}^{\prime \prime}\right)$ situated in the same connected component of the variety $\mathcal{W}$ as the point $y^{(1)}$. Hence $y^{\prime \prime} \in \mathcal{U}_{\varepsilon}^{(1)}$ and $x^{\prime \prime}=s t\left(y^{\prime \prime}\right) \in C_{1}$. Moreover $z_{n-l+1}^{\prime \prime}=z_{n-l+1}^{(1)}$ (see the beginning of the proof of Lemma 8 in Section 2). Therefore, the function $Z_{n-l+1}$ reaches a local maximum $z_{n-l+1}$ on $C_{1}$ both at the point $x$ and at the point $x^{\prime \prime}$. This proves the claim.

After that the algorithm tests, for each of the constructed standard parts of the form $s t\left(y^{\prime}\right)$, whether $s t\left(y^{\prime}\right) \in \mathcal{M}^{\left(\eta_{1}, \ldots, \eta_{n-1}\right)} \mathcal{D}_{0}(\mathcal{R})$, whether $s t\left(y^{\prime}\right)$ is a local extremal point of the function $Z_{n-l+1}$ on the curve $W^{(1)} \cap \tilde{F}^{n}$ and, finally, whether the equalities $\operatorname{st}\left(z_{1}^{\prime}\right)=\eta_{1}, \ldots, s t\left(z_{n-l}^{\prime}\right)=\eta_{n-l}$ are valid (otherwise 
the point $s t\left(y^{\prime}\right)$ is not considered in the following). To achieve this goal, we introduce elements $\varepsilon_{3}, \varepsilon_{4}$ such that $\varepsilon_{3}>0$ is infinitesimal with respect to the field $F$ and $\varepsilon_{4}>0$ is infinitesimal with respect to the field $F\left(\varepsilon_{3}\right)$. One can easily show that the function $Z_{n-l+1}$ reaches a local maximum on the curve $W^{(1)} \cap \tilde{F}^{n}$ at a point $s t\left(y^{\prime}\right)$. Furthermore $\operatorname{st}\left(z_{1}^{\prime}\right)=\eta_{1}, \ldots, s t\left(z_{n-l}^{\prime}\right)=\eta_{n-l}$ and also $s t\left(y^{\prime}\right) \in \mathcal{M}^{\left(\eta_{1}, \ldots, \eta_{n-l}\right)} \mathcal{D}_{0}(\mathcal{R})$ if the following condition is true:

$$
\begin{gathered}
s t\left(y^{\prime}\right) \in\left(\mathcal{M}^{\left(\eta_{1}, \ldots, \eta_{n-l}\right)} \mathcal{D}_{0}(\mathcal{R}) \cap W^{(1)} \cap \tilde{F}^{n}\right) \wedge\left(s t\left(z_{1}^{\prime}\right)\right. \\
\left.=\eta_{1} \wedge \cdots \wedge s t\left(z_{n-l}^{\prime}\right)=\eta_{n-l}\right) \wedge\left(\mathcal{D}_{s t\left(y^{\prime}\right)}\left(\varepsilon_{3}\right) \cap\left(W^{(1)}\right)^{\left(\varepsilon_{3}, \varepsilon_{4}\right)}\right. \\
\left.\cap\left(\tilde{F}\left(\varepsilon_{3}, \varepsilon_{4}\right)\right)^{n} \cap\left\{Z_{n-l+1}=\operatorname{st}\left(z_{n-l+1}^{\prime}\right)+\varepsilon_{4}\right)=\emptyset\right\}
\end{gathered}
$$

where $\left(W^{(1)}\right)^{\left(\varepsilon_{3}, \varepsilon_{4}\right)} \subset\left(\overline{F\left(\varepsilon_{3}, \varepsilon_{4}\right)}\right)^{n}$ denotes the variety determined by the same system of equations (13a) as $W^{(1)}$. The algorithm tests condition (14) with the help of Proposition 2. In a similar manner the algorithm deals with the local minima of the function $Z_{n-l+1}$.

Now we can describe the third procedure for producing the options for elements $\eta_{n-l+1}$. Namely, as $\eta_{n-l+1}$ the algorithm takes $s t\left(z_{n-l+1}^{\prime}\right) \in \tilde{F}$ for every point $s t\left(y^{\prime}\right)$ among those produced above which is a local extremum and is situated in the set $\left\{Z_{1}=\eta_{1} \& \ldots \& Z_{n-l}=\eta_{n-l}\right\} \cap \mathcal{M}^{\left(\eta_{1}, \ldots, \eta_{n-l}\right)} \mathcal{D}_{0}(\mathcal{R})$. As above, for every option for $\eta_{n-l+1}$ the minimal polynomial $\varphi^{\left(\eta_{n-l+1}\right)}$ and the sequence $\mathcal{S}^{\left(\eta_{n-l+1}\right)}$ of signs of the derivatives of $\varphi^{\left(\eta_{n-l+1}\right)}$ are produced. The algorithm considers all the possible curves of the type $W^{(1)}$ (i.e., one-dimensional irreducible components of the variety determined by system (12)). It was shown above that among the produced options of the third procedure are all the local extrema of the function $Z_{n-l+1}$ on $C_{1}$.

Thereupon the algorithm orders (using Proposition 2) all the options for an element $\eta_{n-l+1}$ obtained according to the first, second and third procedures. Consider two adjacent (in the latter ordering) options $\eta^{(1)}<\eta^{(2)}$ and some connected component $C_{2}$ of the intersection $W^{(1)} \cap \tilde{F}^{n} \cap\left\{Z_{1}=\eta_{1} \wedge \cdots \wedge Z_{n-1}=\right.$ $\left.\eta_{n-l} \wedge \eta^{(1)}<Z_{n-l+1}<\eta^{(2)}\right\} \cap \mathcal{M}^{\left(\eta_{1}, \ldots, \eta_{n-l}\right)} \mathcal{D}_{0}(\mathcal{R})$.

LEMMA 12. For any $\eta^{(1)}<\eta<\eta^{(2)}$, an intersection $C_{2} \cap\left\{Z_{n-l+1}=\eta\right\} \neq \emptyset$ is nonempty.

Proof. Assume the contrary. Then the function $Z_{n-l+1}$ has an extremum on $C_{2}$, and thus this extremum is also a local extremum on $W^{(1)} \cap \tilde{F}^{n}$. This contradicts the claim about the third procedure and proves the lemma. $\square$

Now consider a variety $W^{(1)} \subset \bar{F}^{n}$ as produced above, irreducible over $F$ and determined by a system (13a) such that the modified system (13a,b) in 
which the disjunction ranges over all the produced options for elements $\eta_{n-l+1}$ (including the third procedure) is consistent in $\tilde{F}^{n}$. Recall that $W^{(1)}$ is a curve under this specification. The algorithm considers all pairs of adjacent options for $\eta_{n-l+1}$ and for each such a pair $\eta^{(1)}<\eta^{(2)}$ finds, with the help of Proposition 4 (see the Introduction), the connected components of an intersection $W^{(1)} \cap$ $\left\{Z_{1}=\eta_{1} \wedge \cdots \wedge Z_{n-l}=\eta_{n-l} \wedge \eta^{(1)}<Z_{n-l+1}<\eta^{(2)}\right\} \cap \tilde{F}^{n}$. The curve $W^{(1)}$ does not lie in any finite union of the hyperplanes of the kind $\left\{Z_{n-l+1}=\alpha\right\}$ because of Lemma 12. Therefore, an intersection $W^{(1)} \cap\left\{Z_{n-l+1}=\alpha\right\} \subset \bar{F}^{n}$ is either empty or consists of a finite number of points (see above).

Thereupon the algorithm finds all the points of the intersections $W^{(1)} \cap$ $\tilde{F}^{n} \cap\left\{Z_{n}=\eta_{1} \wedge \cdots \wedge Z_{n-l}=\eta_{n-l} \wedge Z_{n-l+1}=\eta_{n-l+1}\right\}$ for all options for $\eta_{n-l+1}$, using Proposition 2. After that the algorithm selects, again using Proposition 2, from among these points all the points which belong to the set $\mathcal{M}^{\left(\eta_{1}, \ldots, \eta_{n-l}\right)} \mathcal{D}_{0}(\mathcal{R})$ (other points are not considered in the following). These selected points, together with the points $u^{(1)}\left(\eta_{1}, \ldots, \eta_{n-l}\right), \ldots, u^{(q)}\left(\eta_{1}, \ldots, \eta_{n-l}\right)$ produced on the previous step of the recursion, and with the points of the type $\sigma\left(\eta_{1}, \ldots, \eta_{n-l}, \eta_{n-l+1}\right)$ produced according to the first procedure, constitute in whole the family of points of the kind $u^{(i)}\left(\eta_{1}, \ldots, \eta_{n-l}, \eta_{n-l+1}\right)$ for different $i$ and different options for $\eta_{n-l+1}$, produced at the current step of recursion.

Denote by $C_{3}$ one of the already found connected components of the intersection $W^{(1)} \cap \tilde{F}^{n} \cap\left\{Z_{1}=\eta_{1} \wedge \cdots \wedge Z_{n-l}=\eta_{n-l} \wedge \eta^{(1)}<Z_{n-l+1}<\eta^{(2)}\right\}$. (Actually, the algorithm considers all the pairs of adjacent options $\eta^{(1)}<\eta^{(2)}$ for $\eta_{n-l+1}$ and all connected components $C_{3}$.) Observe that any point of the intersection of the closure $\bar{C}_{3}$ (in the topology of the space $\tilde{F}^{n}$ with the base of all open balls) with two planes $\left\{Z_{1}=\eta_{1} \wedge \cdots \wedge Z_{n-l}=\eta_{n-l} \wedge Z_{n-l+1}=\eta^{(1)}\right\}$ and $\left\{Z_{1}=\right.$ $\left.\eta_{1} \wedge \cdots \wedge Z_{n-l}=\eta_{n-l} \wedge Z_{n-l+1}=\eta^{(2)}\right\}$ is contained among the produced points of the kind $u^{(i)}\left(\eta_{1}, \ldots, \eta_{n-l}, \eta_{n-l+1}\right)$ (provided that $\mathcal{C}_{3} \subset \mathcal{M}^{\left(\eta_{1}, \ldots, \eta_{n-l}\right)} \mathcal{D}_{0}(\mathcal{R})$ ). In order to test whether a point $u=u^{(i)}\left(\eta_{1}, \ldots, \eta_{n-l}, \eta_{n-l+1}\right)$ lies in the closure $\bar{C}_{3}$, it suffices to check the nonemptiness of the intersection $\mathcal{D}_{u}\left(\varepsilon_{3}\right) \cap C_{3}^{\left(\varepsilon_{3}\right)}$ (recall that the element $\varepsilon_{3}>0$ is infinitesimal with respect to the field $F$ ) where $C_{3}^{\left(\varepsilon_{3}\right)} \subset\left(\tilde{F}\left(\varepsilon_{3}\right)\right)^{n}$ is a semialgebraic set determined by the same quantifier-free formulas as $C_{3}$ (see Proposition 4). The algorithm checks the nonemptiness of this intersection with the aid of Proposition 2. If some point (and hence any point of $\bar{C}_{3}$, because of the properties of $\mathcal{R}$ discussed at the beginning of this section) does not lie in the set $\mathcal{M}^{\left(\eta_{1}, \ldots, \eta_{n-l}\right)} \mathcal{D}_{0}(\mathcal{R})$ then the connected component $C_{3}$ is not considered in the following.

By virtue of the recursive hypothesis (see the beginning of this section), for each option for $\eta_{n-l+1}$, the algorithm can construct a skeleton $\Sigma^{\left(\eta_{1}, \ldots, \eta_{n-l}, \eta_{n-l+1}\right)}$ containing all the points of the kind $u^{(i)}\left(\eta_{1}, \ldots, \eta_{n-l}, \eta_{n-l+1}\right)$ for the union 
of all connected components of the variety $\left\{h^{\left(\eta_{1}, \ldots, \eta_{n-l}\right)}\left(\eta_{1}, \ldots, \eta_{n-l}, \eta_{n-l+1}\right.\right.$, $\left.\left.Y_{1}, \ldots, Y_{l-1}\right)=0\right\} \subset\left(\tilde{F}^{n} \cap\left\{Z_{1}=\eta_{1} \wedge \ldots \wedge Z_{n-l}=\eta_{n-l} \wedge Z_{n-l+1}=\eta_{n-l+1}\right\}\right)$ which lie in the set $\mathcal{M}^{\left(\eta_{1}, \ldots, \eta_{n-l}\right)} \mathcal{D}_{0}(\mathcal{R})$. Then as $\Sigma^{\left(\eta_{1}, \ldots, \eta_{n-l}\right)}$ we shall take the union of the curves $\Sigma^{\left(\eta_{1}, \ldots, \eta_{n-l}, \eta_{n-l+1}\right)}$ for all the options for $\eta_{n-l+1}$ together with the union of all produced curves of the type $C_{3}$. In the next section we describe how the algorithm represents $\Sigma^{\left(\eta_{1}, \ldots, \eta_{n-l}\right)}$. We will also prove that $\Sigma^{\left(\eta_{1}, \ldots, \eta_{n-l}\right)}$ is a skeleton for the union of all the connected components of the variety $\left\{h^{\left(\eta_{1}, \ldots, \eta_{n-l-1}\right)}\left(\eta_{1}, \ldots, \eta_{n-l}, Y_{1}, \ldots, Y_{l}\right)=0\right\} \subset\left(\tilde{F}^{n} \cap\left\{Z_{1}=\eta_{1} \wedge \cdots \wedge Z_{n-l}=\right.\right.$ $\left.\left.\eta_{n-l}\right\}\right)$ which lie in the set $\mathcal{M}^{\left(\eta_{1}, \ldots, \eta_{n-l-1}\right)} \mathcal{D}_{0}(\mathcal{R})$.

\section{Correctness of the algorithm for constructing a skeleton}

First, we prove by induction on $l$ that $\Sigma^{\left(\eta_{1}, \ldots, \eta_{n-l}\right)}$ passes through all points of the kind $u^{(i)}\left(\eta_{1}, \ldots, \eta_{n-l}\right)$. It suffices to check the basis of the induction for the case of the plane $(l=2)$. This follows from the observation that $\Sigma^{\left(\eta_{1}, \ldots, \eta_{n-2}\right)}$ coincides with the intersection $\left\{h^{\left(\eta_{1}, \ldots, \eta_{n-2}\right)}\left(\eta_{1}, \ldots, \eta_{n-2}, Z_{n-1}, Y_{1}=0\right\} \cap\left\{Z_{1}=\right.\right.$ $\left.\eta_{1}, \ldots, Z_{n-2}=\eta_{n-2}\right\} \cap \mathcal{M}^{\left(\eta_{1}, \ldots, \eta_{n-3}\right)} \mathcal{D}_{0}(\mathcal{R})$ (see Lemma 10 ).

According to the recursive hypothesis, a skeleton $\Sigma^{\left(\eta_{1}, \ldots, \eta_{n-l}, \eta_{n-l+1}\right)}$ consists of the union of some family of connected closed semialgebraic curves $C^{(i)}$ for $1 \leq i \leq t$, each of which is determined by a certain quantifier-free formula. In addition, the graph $F^{\left(\eta_{1}, \ldots, \eta_{n-l}, \eta_{n-l+1}\right)}$ is produced whose $t$ vertices correspond bijectively to the curves $C^{(i)}$ for $1 \leq i \leq t$. Two vertices are linked by an edge if the corresponding curves have common end points (i.e., the points from $\left.\bar{C}^{(i)} \backslash C^{(i)}\right)$. Thus the algorithm represents $\Sigma^{\left(\eta_{1}, \ldots, \eta_{n-l}, \eta_{n-l+1}\right)}$ by the formula determining $C^{(i)}$ for $1 \leq i \leq t$, and also by the graph $F^{\left(\eta_{1}, \ldots, \eta_{n-l}, \eta_{n-l+1}\right)}$. When $n-l+1=0$ we denote the graph by $F$.

On the current recursive step the algorithm adds to the union of

$$
\Sigma^{\left(\eta_{1}, \ldots, \eta_{n-l}, \eta_{n-l+1}\right)},
$$

over all the options for $\eta_{n-l+1}$, the closures $\bar{C}_{2}$ of all the curves of the type $C_{3}$ as in the previous section. As a result we obtain $\Sigma^{\left(\eta_{1}, \ldots, \eta_{n-l}\right)}$. Next, the algorithm produces a graph $F^{\left(\eta_{1}, \ldots, \eta_{n-l}\right)}$ adding to the union of all graphs of the kind $F^{\left(\eta_{1}, \ldots, \eta_{n-l}, \eta_{n-l+1}\right)}$ over all the options for $\eta_{n-l+1}$, the new vertices corresponding to the curves of the type $\bar{C}_{3}$. Also, we add a new edge in $F^{\left(\eta_{1}, \ldots, \eta_{n-l}\right)}$ between a new vertex corresponding to a curve of the type $\bar{C}_{3}$ and some other vertex if the corresponding curves have a common endpoint (it is of the type $u^{(i)}\left(\eta_{1}, \ldots, \eta_{n-l}\right.$, $\left.\eta_{n-l+1}\right)$ ). These new edges can be computed using the algorithm of the previous section. 
LEMMA 13. $\Sigma^{\left(\eta_{1}, \ldots, \eta_{n-l}\right)}$ is a skeleton for the union of the connected components of the variety

$$
\begin{aligned}
& \left\{h^{\left(\eta_{1}, \ldots, \eta_{n-l-1}\right)}\left(\eta_{1}, \ldots, \eta_{n-l}, Y_{1}, \ldots, Y_{l}\right)=0\right\} \\
& \subset\left(\tilde{F}^{n} \cap\left\{Z_{1}=\eta_{1} \wedge \cdots \wedge Z_{n-l}=\eta_{n-l}\right\}\right)
\end{aligned}
$$

which lie in the set $M^{\left(\eta_{1}, \ldots, \eta_{n-l-1}\right)} \mathcal{D}_{0}(\mathcal{R})$.

Proof. Consider one of these connected components $\mathcal{W}$. If

$$
\mathcal{W} \subset\left\{Z_{1}=\eta_{1} \wedge \cdots \wedge Z_{n-l}=\eta_{n-l} \wedge Z_{n-l+1}=\eta_{n-l+1}\right\}
$$

for a certain option for $\eta_{n-l+1}$, then $\Sigma^{\left(\eta_{1}, \ldots, \eta_{n-l}, \eta_{n-l+1}\right)} \cap \mathcal{W}$ is a skeleton for $\mathcal{W}$ because of the recursive hypothesis. By the same token

$$
\Sigma^{\left(\eta_{1}, \ldots, \eta_{n-l}\right)} \cap \mathcal{W}=\Sigma^{\left(\eta_{1}, \ldots, \eta_{n-l}, \eta_{n-l+1}\right)} \cap \mathcal{W}
$$

is also a skeleton for $\mathcal{W}$. Assume now that $\mathcal{W}$ is not situated in any plane of the considered form for any option for $\eta_{n-l+1}$. Lemma 11(2) implies nonemptiness of an intersection $\mathcal{W} \cap \Sigma^{\left(\eta_{1}, \ldots, \eta_{n-l}\right)} \neq \emptyset$.

Our purpose is to prove that the latter intersection is connected. Denote by $\mathcal{V}_{1}, \ldots, \mathcal{V}_{\mu}$ the connected components of the difference $\mathcal{W} \backslash \bigcup_{\eta_{n-l+1}}\left\{Z_{n-l+1}=\right.$ $\left.\eta_{n-l+1}\right\}$, where the union ranges over all the options for $\eta_{n-l+1}$. Denote by $\mathcal{U}_{1}, \ldots, \mathcal{U}_{\rho}$ the connected components of the intersections $\mathcal{W}\left\{\left\{Z_{n-l+1}=\eta_{n-l+1}\right\}\right.$ for all the options for $\eta_{n-l+1}$. We consider a pair $\eta^{(1)}<\eta^{(2)}$ of adjacent options for $\eta_{n-1+1}$. Suppose that some connected component $\mathcal{V}_{i}$ lies in a set $\left\{\eta^{(1)}<\right.$ $\left.Z_{n-l+1}<\eta^{(2)}\right\}$. Then, for arbitrary $\eta^{(3)}, \eta^{(4)}$ such that $\eta^{(1)}<\eta^{(3)}<\eta^{(4)}<\eta^{(2)}$, the intersections $\mathcal{V}_{i} \cap\left\{Z_{n-l+1}=\eta^{(3)}\right\}$ and $\mathcal{V}_{i} \cap\left\{Z_{n-l+1}=\eta^{(4)}\right\}$ are homeomorphic since between $\eta^{(1)}$ and $\eta^{(2)}$ there are no critical values of the function $Z_{n-l+1}$ on the nonsingular variety $\mathcal{V}_{i}$; see system $(10 \mathrm{a}, \mathrm{b})$ (Hirsch 1976). Therefore, $\mathcal{V}_{i}$ is homeomorphic to a cylinder $\left(\mathcal{V}_{i} \cap\left\{Z_{n-l+1}=\eta^{(3)}\right\}\right) \times(0,1)$; hence the base $\mathcal{V}_{i} \cap\left\{Z_{n-l+1}=\eta^{(3)}\right\}$ of the cylinder is connected. One can show (using the transfer principle) that the intersection of the closure $\overline{\mathcal{V}}_{i}$ with the hyperplane $\left\{Z_{n-l+1}=\eta^{(1)}\right\}$ is connected (taking into account the boundness of $\overline{\mathcal{V}}_{i}$ ). Because of that, $\overline{\mathcal{V}}_{i} \cap\left\{Z_{n-l+1}=\eta^{(1)}\right\} \subset \mathcal{U}_{j}$ for a suitable unique index $j$ (similarly for the hyperplane $\left\{Z_{n-l+1}=\eta^{(2)}\right\}$ ).

Introduce now the following bipartite graph $\mathcal{H}$ whose vertices correspond bijectively to the connected components $\mathcal{V}_{i}, \mathcal{U}_{j}(1 \leq i \leq \mu, 1 \leq j \leq \rho)$. An edge linking two vertices corresponding to $\mathcal{V}_{i}, \mathcal{U}_{j}$ is drawn if $\overline{\mathcal{V}}_{i} \cap \mathcal{U}_{j} \neq \emptyset$. A vertex of $\mathcal{H}$ corresponding to $\mathcal{V}_{i}$ has exactly two adjacent vertices, corresponding to the 
intersections of $\overline{\mathcal{V}}_{i}$ with the hyperplanes $\left\{Z_{n-l+1}=\eta^{(1)}\right\}$ and $\left\{Z_{n-l+1}=\eta^{(2)}\right\}$, respectively. Since $\mathcal{W}$ is connected, the graph $\mathcal{H}$ is also connected.

For two arbitrary points $x, y \in\left(\mathcal{W} \cap \Sigma^{\left(\eta_{1}, \ldots, \eta_{n-l}\right)}\right)$ we now prove the existence of a (connected) path $\mathcal{P} \subset \Sigma^{\left(\eta_{1}, \ldots, \eta_{n-l}\right)}$ linking $x$ and $y$. Let $x$ and $y$ belong to some connected components of type either $\mathcal{U}_{j}$ or $\mathcal{V}_{i}$. Draw a path $\mathcal{P}$ in the graph $\mathcal{H}$ linking the vertices corresponding to the connected components containing $x$ and $y$, respectively. We argue by induction on the length of the path $\mathcal{P}$. The basis of induction concerns the case when $x$ and $y$ lie in the same connected component. If this component is $\mathcal{U}_{j} \subset\left\{Z_{n-l+1}=\eta^{(1)}\right\}$ then we apply the inductive hypothesis to the skeleton $\Sigma^{\left(\eta_{1}, \ldots, \eta_{n-l}, \eta^{(1)}\right)}$. Otherwise, if this component is $\mathcal{V}_{i}$ (let $\mathcal{V}_{i} \subset\left\{\eta^{(1)}<Z_{n-l+1}<\eta^{(2)}\right\}$ ), then consider the connected components $C_{x}, C_{y} \subset\left(\mathcal{V}_{i} \cap \Sigma^{\left(\eta_{1}, \ldots, \eta_{n-l}\right)}\right)$ of the intersections of the curves of the kind $W^{(1)} \cap \tilde{F}^{n}$ (see $\left.(13 \mathrm{a}, \mathrm{b})\right)$ with the set $\left\{\eta^{(1)}<Z_{n-l+1}<\eta^{(2)}\right\}$, such that $x \in C_{x}$ and $y \in C_{y}$. Lemma 12 implies the existence of the points $x_{1} \in\left(\bar{C}_{x} \cap\left\{Z_{n-l+1}=\eta^{(1)}\right\}\right), y_{1} \in\left(\bar{C}_{y} \cap\left\{Z_{n-l+1}=\eta^{(1)}\right\}\right)$, and $x_{1}, y_{1} \in$ $\left(\overline{\mathcal{V}}_{i} \cap\left\{Z_{n-l+1}=\eta^{(1)}\right\} \cap \Sigma^{\left(\eta_{1}, \ldots, \eta_{n-l}\right)}\right) \subset \mathcal{U}_{j}$ for a suitable index $j$ (see above). The intersection

$$
\Sigma^{\left(\eta_{1}, \ldots, \eta_{n-l}\right)} \cap \mathcal{U}_{j}=\Sigma^{\left(\eta_{1}, \ldots, \eta_{n-l}, \eta^{(1)}\right)} \cap \mathcal{U}_{j}
$$

is connected by the recursive hypothesis, and therefore the points $x$ and $y$ can be linked by a path in $\Sigma^{\left(\eta_{1}, \ldots, \eta_{n-1}\right)}$. This proves the basis of induction.

To prove the inductive step, consider an extreme edge in the path $\mathcal{P}$. Let it correspond (see the graph $\mathcal{H}$ ) to a pair of connected components $\mathcal{V}_{i}, \mathcal{U}_{j}$ (let $\left.\mathcal{V}_{i} \subset\left\{\eta^{(1)}<Z_{n-l+1}<\eta^{(2)}\right\}\right)$. Consider two cases. In the first case $y \in \mathcal{V}_{i}$. As above, consider a connected curve $C_{y} \subset\left(\Sigma^{\left(\eta_{1}, \ldots, \eta_{n-l}\right)} \cap \mathcal{V}_{i}\right)$ such that $y \in C_{y}$. Then an intersection $\bar{C}_{y} \cap \mathcal{U}_{j} \neq \emptyset$ is not empty. Pick out a certain point $y_{1} \in \bar{C}_{y} \cap \mathcal{U}_{j}$ and, as an extreme link of the path $\mathcal{P}$ under construction, take a connected curve contained in $\bar{C}_{y}$ and linking $y_{1}$ and $y$. The points $x$ and $y_{1}$ can be linked in $\Sigma^{\left(\eta_{1}, \ldots, \eta_{n-l}\right)}$ by the inductive hypothesis.

In the second case $y \in \mathcal{U}_{j}$. There exists a connected component $C_{4}$ of the intersection of a certain curve of the kind $W^{(1)} \cap \tilde{F}^{n}$ with the set $\left\{\eta^{(1)}<\right.$ $\left.Z_{n-l+1}<\eta^{(2)}\right\}$ such that $C_{4} \subset\left(\mathcal{V}_{i} \cap \Sigma^{\left(\eta_{1}, \ldots, \eta_{n-l}\right)}\right)$ by virtue of Lemma 11(2). Lemma 12 implies that $\bar{C}_{4} \cap \mathcal{U}_{j} \neq \emptyset$ is not empty. Pick out a certain point $y_{2} \in \bar{C}_{4} \cap \mathcal{U}_{j}$. By the recursive hypothesis, for $\Sigma^{\left(\eta_{1}, \ldots, \eta_{n-l}, \eta\right)}$ (here either $\eta=\eta^{(1)}$ or $\left.\eta=\eta^{(2)}\right)$, the points $y$ and $y_{2}$ can be linked by a path in $\Sigma^{\left(\eta_{1}, \ldots, \eta_{n-l}\right)} \cap \mathcal{U}_{j}$. An arbitrary point $y_{3} \in C_{4}$ can be linked with $y_{2}$ by a path in $\bar{C}_{4} \subset \Sigma^{\left(\eta_{1}, \ldots, \eta_{n-1}\right)}$. Finally, one can link $x$ and $y_{3}$ by a path in $\Sigma^{\left(\eta_{1}, \ldots, \eta_{n-l}\right)}$ using the inductive hypothesis. This yields a path $\mathcal{P}$ linking $x$ and $y$ in $\Sigma^{\left(\eta_{1}, \ldots, \eta_{n-l}\right)}$. Thus, the intersection $\mathcal{W} \cap \Sigma^{\left(\eta_{1}, \ldots, \eta_{n-l}\right)}$ is connected. 
The description of the recursive construction of a skeleton $\Sigma$ for the hypersurface $\{h=0\} \cap \mathcal{D}_{0}(\mathcal{R})$ containing the points $u^{(1)}, \ldots, u^{(p)}$ (see the beginning of Section 3) and the proof of correctness of the algorithm are now completed. The number of connected components of the hypersurface $\{h=0\} \cap \mathcal{D}_{0}(\mathcal{R})$ coincides with the number of connected components of the skeleton $\Sigma$ and, by the same token, with the number of connected components of the graph $F$ (see the beginning of the section). Moreover, one can easily test whether two points $u^{(1)}, u^{(2)}$ belong to the same connected component of the hypersurface $\{h=0\} \cap \mathcal{D}_{0}(\mathcal{R})$, using the graph $F$.

This completes the design of the algorithm required in the main theorem (see the Introduction). In the next section we shall analyze the complexity of the algorithm.

\section{Complexity analysis of the algorithm}

First, we turn to the complexity analysis of the algorithm described in Section 3. Taking into account that this algorithm has a recursive structure, and each of its steps consists of performing several arithmetic operations, it suffices to estimate a priori the bit lengths of the data over which the operations are accomplished, the number of operations at one step, and finally, the total number of the steps in the recursion.

Now we proceed to a priori estimate the intermediate data in the algorithm. By recursion on $(n-l)$ we will produce a certain family of the formula of the first-order theory of the field $\tilde{F}$ which we will use to help estimate the parameters of the part of the skeleton constructed on the $(n-l)$-th recursive step of the algorithm. At the beginning of the current step of the algorithm the points

$$
u^{(1)}\left(\eta_{1}, \ldots, \eta_{n-l}\right), \ldots, u^{(q)}\left(\eta_{1}, \ldots, \eta_{n-l}\right)
$$

are produced. Fixing $j$ for now, for each of these points $u^{(j)}\left(\eta_{1}, \ldots, \eta_{n-l}\right)$ we assume that some formula of the theory of the field $\tilde{F}$ (with three quantifier alternations) of the following form is already produced:

$$
\varphi^{(j)}=\exists T_{1,1 \ldots} \exists T_{1, \tau_{1}} \forall T_{2,1 \ldots} \forall T_{2, \tau_{2}} \exists T_{3,1 \ldots} \exists T_{3, \tau_{3}}\left(\psi^{(j)}\right) .
$$

Here $\psi^{(j)}$ is a quantifier-free formula with the atomic subformulae of the kind $(f \geq 0)$, where $f \in F\left[T_{1,1}, \ldots, T_{1, \tau_{1}}, T_{2,1}, \ldots, T_{2, \tau_{2}}, T_{3,1}, \ldots, T_{3, \tau_{3}}, Z_{1}, \ldots, Z_{n-l}\right.$, $\left.Y_{1}, \ldots, Y_{l}\right]$. Furthermore, every point from the semialgebraic set $\left\{\varphi^{(j)}\right\} \cap\left\{Z_{1}=\right.$ $\left.\eta_{1} \wedge \cdots \wedge Z_{n-l}=\eta_{n-l}\right\} \subset \tilde{F}^{n}$ is a null-dimensional connected component (in other words, an isolated point) of the semialgebraic set $\left\{\varphi^{(j)}\right\}$, and moreover the point $u^{(j)}\left(\eta_{1}, \ldots, \eta_{n-l}\right) \in\left(\left\{\varphi^{(j)}\right\} \cap\left\{Z_{1}=\eta_{1} \wedge \cdots \wedge Z_{n-l}=\eta_{n-l}\right\}\right)$. 
Thereupon we produce a certain formula $\varphi$ of one of three types, according to the three procedures producing options for an element $\eta_{n-l+1}$ (see Section $3)$. According to the first procedure as $\varphi$ we take the formula:

$$
\begin{gathered}
\exists Y_{l, 1, . .} \exists Y_{l, l-1}\left(h^{\left(\eta_{1}, \ldots, \eta_{n-l}\right)}\left(Z_{1}, \ldots, Z_{n-l+1}, Y_{l, 1}, \ldots, Y_{l, l-1}\right)\right. \\
=\frac{\partial h^{\left(\eta_{1}, \ldots, \eta_{n-l}\right)}\left(Z_{1}, \ldots, Z_{n-l+1}, Y_{l, 1}, \ldots, Y_{l, l-1}\right)}{\partial Y_{l, 1}} \\
=\cdots=\frac{\partial h^{\left(\eta_{1}, \ldots, \eta_{n-l}\right)}\left(Z_{1}, \ldots, Z_{n-l+1}, Y_{l, 1}, \ldots, Y_{l, l-1}\right)}{\partial Y_{l, l-1}}=0 \\
\left.\wedge\left(Z_{1}, \ldots, Z_{n-l+1}, Y_{l, 1}, \ldots, Y_{l, l-1}\right) \in \mathcal{M}^{\left(\eta_{1}, \ldots, \eta_{n-l}\right)} \mathcal{D}_{0}(\mathcal{R})\right) .
\end{gathered}
$$

Observe that for $Z_{1}=\eta_{1}, \ldots, Z_{n-l}=\eta_{n-l}$, the quantifier-free part of formula $(15)_{I}$ has the same finite set of solutions (as a system of inequalities) as the set of solutions of system $(10 \mathrm{a}, \mathrm{b})$ which lie in the set $\mathcal{M}^{\left(\eta_{1}, \ldots, \eta_{n-l}\right)} \mathcal{D}_{0}(\mathcal{R})$. Therefore, the set of the values of the coordinate $Z_{n-l+1}$ of all points satisfying (15) (for $Z_{1}=\eta_{1}, \ldots, Z_{n-l}=\eta_{n-l}$ ) coincides with the set of all options for $\eta_{n-l+1}$ produced according to the first procedure.

According to the second procedure, denote by $\tilde{\varphi}^{(j)}$ the formula obtained from $\varphi^{(j)}$ by means of the linear transformation of the variables $\left(Y_{1}, \ldots, Y_{l}\right)^{T} \rightarrow$ $\mathcal{B}^{-1}\left(\bar{Y}_{1}, \ldots, \bar{Y}_{l}\right)^{T}$ (see Lemma 9 and the construction just after it). Furthermore, denote by $\bar{\varphi}^{(j)}$ the formula obtained from $\bar{\varphi}^{(j)}$ by replacing variables $\bar{Y}_{1}, \ldots, \bar{Y}_{l}$ by $Z_{n-l+1}, Y_{l, 1}, \ldots, Y_{l, l-1}$, respectively. As $\varphi$ take the formula:

$$
\exists Y_{l, 1 \ldots} \exists Y_{l, l-1}\left(\tilde{\varphi}^{(j)}\right) .
$$

Note that the point $\left(\eta_{1}, \ldots, \eta_{n-l}, u_{1}^{(j)}+\sum_{2 \leq i \leq l} t_{i} u_{i}^{(j)}\right)$ is a null-dimensional connected component of the semialgebraic set determined by formula $(15)_{I I}$ (recall that the point $\left.u^{(j)}\left(\eta_{1}, \ldots, \eta_{n-l}\right)=\left(\eta_{1}, \ldots, \eta_{n-l}, u_{1}^{(j)}, \ldots, u_{l}^{(j)}\right)\right)$.

Denote the following formula by $\Omega^{\left(\eta_{1}, \ldots, \eta_{n-l}\right)}$ (see (12)):

$$
\begin{aligned}
h^{\left(\eta_{1}, \ldots, \eta_{n-l}\right)} & =\left(\frac{\partial h^{\left(\eta_{1}, \ldots, \eta_{n-l}\right)}}{\partial Y_{1}}\right)^{2}-\frac{\lambda_{1}}{N_{2} l} \Delta \\
& =\cdots=\left(\frac{\partial h^{\left(\eta_{1}, \ldots, \eta_{n-l}\right)}}{\partial Y_{l-2}}\right)^{2}-\frac{\lambda_{l-2}}{N_{2} l} \Delta=0 .
\end{aligned}
$$

According to the third procedure, as $\varphi$ we shall take either the following formula, which we denote by $(15)_{I I I \max }$ :

$$
\exists Y_{l, 1 \ldots} \exists Y_{l, l-1} \exists R \forall R^{(1)} \exists Z_{n-l+1}^{(1)} \exists Y_{l, 1 \ldots}^{(1)} \exists Y_{l, l-1}^{(i)} \exists Z_{n-l+1}^{(2)}
$$




$$
\begin{aligned}
\exists Y_{l, 1 \ldots}^{(2)} \exists Y_{l, l-1}^{(2)}( & \left(0<R^{(1)} \leq R\right) \Rightarrow\left(\left.\Omega^{\left(\eta_{1}, \ldots, \eta_{n-l}\right)}\right|_{\left(Z_{1}, \ldots, Z_{n-l}, Z_{n-l+1}, Y_{l, 1}, \ldots, Y_{l, l-1}\right)}\right. \\
& \left.\wedge \Omega^{\left(\eta_{1}, \ldots, \eta_{n-l}\right)}\right|_{\left(Z_{1}, \ldots, Z_{n-l}, Z_{n-l+1}^{(1)}, Y_{l, 1}^{(1)}, \ldots, Y_{l, l-1}^{(1)}\right)} \\
& \left.\wedge \Omega^{\left(\eta_{1}, \ldots, \eta_{n-l}\right)}\right|_{\left(Z_{1}, \ldots, Z_{n-l}, Z_{n-l+1}^{(2)}, Y_{l, 1}^{(2)}, \ldots, Y_{l, l-1}^{(2)}\right)} \\
& \wedge \|\left(Z_{1}, \ldots, Z_{n-l}, Z_{n-l+1}, Y_{l, 1}, \ldots, Y_{l, l-1}\right) \\
- & \left(Z_{1}, \ldots, Z_{n-l}, Z_{n-l+1}^{(1)}, Y_{l, 1}^{(1)}, \ldots, Y_{l, l-1}^{(1)} \|^{2}=R^{(1)}\right. \\
& \wedge \|\left(Z_{1}, \ldots, Z_{n-l}, Z_{n-l+1}, Y_{l, 1}, \ldots, Y_{l, l-1}\right) \\
- & \left(Z_{1}, \ldots, Z_{n-l}, Z_{n-l+1}^{(2)}, Y_{l, 1}^{(2)}, \ldots, Y_{l, l-1}^{(2)}\right) \|^{2}=R^{(1)} \\
\wedge\left(\left(Z_{1}, \ldots,\right.\right. & \left.\left.Z_{n-l}, Z_{n-l+1}, Y_{l, 1}, \ldots, Y_{l, l-1}\right) \in \mathcal{M}^{\left(\eta_{1}, \ldots, \eta_{n-l}\right)} \mathcal{D}_{0}(\mathcal{R})\right) \\
& \wedge\left(\left(Z_{1}, \ldots, Z_{n-l}, Z_{n-l+1}^{(1)}, Y_{l, 1}^{(1)}, \ldots, Y_{l, l-1}^{(1)}\right)\right. \\
& \left.\neq\left(Z_{1}, \ldots, Z_{n-l}, Z_{n-l+1}^{(2)}, Y_{l, 1}^{(2)}, \ldots, Y_{l, l-1}^{(2)}\right)\right) \\
\wedge & \left.\left.\left(Z_{n-l+1}^{(1)} \leq Z_{n-l+1}\right) \&\left(Z_{n-l+1}^{(2)} \leq Z_{n-l+1}\right)\right)\right)
\end{aligned}
$$

(here the vertical line is used for the notation of substitution), or the similar formula $(15)_{I I I \min }$, obtained from $(15)_{I I I \max }$ by replacing the latter inequalities $\left(Z_{n-l+1}^{(1)} \leq Z_{n-l+1}\right)$ and $\left(Z_{n-l+1}^{(2)} \leq Z_{n-l+1}\right)$ by the opposite ones: $\left(Z_{n-l+1}^{(1)} \geq Z_{n-l+1}\right)$ and $\left(Z_{n-l+1}^{(2)} \geq Z_{n-l+1}\right)$ respectively. Observe that for $Z_{1}=\eta_{1}, \ldots, Z_{n-l}=\eta_{n-l}$ the set $\mathcal{L}$ of the values of the coordinate $Z_{n-l+1}$ (excepting the options for $\eta_{n-l+1}$ produced according to the first procedure) of the points satisfying either $(15)_{I I I \max }$ or $(15)_{I I I \min }$ is finite and coincides with the union of two following sets. The first set consists of the local extrema of the coordinate $Z_{n-l+1}$ on the curve (see Lemma 10):

$$
\begin{aligned}
\left\{h^{\left(\eta_{1}, \ldots, \eta_{n-l}\right)}\right. & =\left(\frac{\partial h^{\left(\eta_{1}, \ldots, \eta_{n-l}\right)}}{\partial Y_{1}}\right)^{2}-\frac{\lambda_{1}}{N_{2} l} \Delta \\
& \left.=\cdots=\left(\frac{\partial h^{\left(\eta_{1}, \ldots, \eta_{n-l}\right)}}{\partial Y_{l-2}}\right)^{2}-\frac{\lambda_{l-2}}{N_{2} l} \Delta=0\right\} \\
& \cap\left\{Z_{1}=\eta_{1} \wedge \cdots \wedge Z_{n-l}=\eta_{n-l}\right\} \cap \mathcal{M}^{\left(\eta_{1}, \ldots, \eta_{n-l}\right)} \mathcal{D}_{0}(\mathcal{R}) \backslash F
\end{aligned}
$$

and the second set consists of the values of the coordinate $Z_{n-l+1}$ at the points of the "self-crossings" of the latter curve. Therefore $\mathcal{L}$ contains the set of 
options for $\eta_{n-l+1}$ produced according to the third procedure (namely, the first mentioned set of local extrema, possibly with some added options for $\eta_{n-1+1}$ according to the first procedure).

Recall that in the recursion step described in Section 3, the algorithm yields points of the kind $u^{(i)}\left(\eta_{1}, \ldots, \eta_{n-l}, \eta_{n-l+1}\right)$. For each of them we specify a formula $\varphi_{0}^{(i)}$ similar to $\varphi^{(j)}$. These points are obtained in three ways according to three procedures (see Section 3 ). First, there are the points satisfying system $(10 \mathrm{a}, \mathrm{b})$. In this case the corresponding formula is

$$
\begin{aligned}
\varphi_{0}^{(i)} & =\exists Y_{l, 1 \ldots}^{(3)} \exists Y_{l, l}^{(3)}\left(\left.\varphi^{(j)}\right|_{\left(Z_{1}, \ldots, Z_{n-l}, Y_{l, 1}^{(3)}, \ldots, Y_{l, l}^{(3)}\right)}\right) \\
& \wedge\left(h^{\left(\eta_{1}, \ldots, \eta_{n-l}\right)}=\frac{\partial h^{\left(\eta_{1}, \ldots, \eta_{n-l}\right)}}{\partial Y_{1}}=\cdots=\frac{\partial h^{\left(\eta_{1}, \ldots, \eta_{n-l}\right)}}{\partial Y_{l-1}}=0\right) .
\end{aligned}
$$

Second, in the set of indicated points the algorithm includes the points of the kind $u^{(j)}\left(\eta_{1}, \ldots, \eta_{n-l}\right)$ produced in the previous step of the recursion. As corresponding formula $\varphi_{0}^{(i)}$ in the second case we take $\tilde{\varphi}^{(j)}$ (see above).

Third, the algorithm obtains the points of intersection of the curves of the type $W^{(1)} \cap \tilde{F}^{n}$ (see (13)) with the planes $\left\{Z_{1}=\eta_{1} \wedge \cdots \wedge Z_{n-l}=\eta_{n-l} \wedge Z_{n-l+1}=\right.$ $\left.\eta_{n-l+1}\right\}$ for all the options for $\eta_{n-l+1}$. In the third case as $\varphi_{0}^{(i)}$ we take the following formula:

$$
\begin{gathered}
\exists Y_{l, 1 \ldots}^{(3)} \exists Y_{l, l}^{(3)}\left(\left.\varphi^{(j)}\right|_{\left(Z_{1}, \ldots, Z_{n-l}, Y_{l, 1}^{(3)}, \ldots, Y_{l, l}^{(3)}\right)}\right) \\
\wedge \varphi \wedge \forall \mathcal{R}^{(4)} \exists Z_{n-l+1}^{(4)} \exists Y_{l, 1 \ldots}^{(4)} \exists Y_{l, l-1}^{(4)}\left(\left(\mathcal{R}^{(4)}>0\right)\right. \\
\Rightarrow\left(( Z _ { n - l + 1 } ^ { ( 4 ) } \neq Z _ { n - l + 1 } ) \wedge \left(\|\left(Z_{1}, \ldots, Z_{n-l}, Z_{n-l+1}, Y_{1}, \ldots, Y_{l-1}\right)\right.\right. \\
\left.-\left(Z_{1}, \ldots, Z_{n-l}, Z_{n-l+1}^{(4)}, Y_{l, 1}^{(4)}, \ldots, Y_{l, l-1}^{(4)}\right) \|^{2} \leq \mathcal{R}^{(4)}\right) \\
\wedge\left(h^{\left(\eta_{1}, \ldots, \eta_{n-l}\right)}=\left(\frac{\partial h^{\left(\eta_{1}, \ldots, \eta_{n-l}\right)}}{\partial Y_{1}}\right)^{2}-\frac{\lambda_{1}}{N_{2} l} \Delta=\ldots=\left(\frac{\partial h^{\left(\eta_{1}, \ldots, \eta_{n-l}\right)}}{\partial Y_{l-2}}\right)^{2}\right. \\
\left.\left.-\frac{\lambda_{l-2}}{N_{2} l} \Delta=0\right)\left.\right|_{\left(Z_{1}, \ldots, Z_{n-l}, Z_{n-l+1}^{(4)}, Y_{l, 1}^{(4)}, \ldots, Y_{l, l-1}^{(4)}\right)}\right) .
\end{gathered}
$$

This formula is true for any point situated on the planes of the kind $\left\{Z_{1}=\right.$ $\left.\eta_{1} \wedge \cdots \wedge Z_{n-l}=\eta_{n-l} \& Z_{n-l+1}=\eta_{n-l+1}\right\}$ which belongs to the closure of a curve of the type $C_{2}$ (see Section 3). Note that the number of these points is finite (see Lemma 12). Moreover, each of these points is a connected component of 
the semialgebraic set determined by the latter formula $\varphi_{0}^{(i)}$, taking into account the properties of the formula $\varphi^{(j)}, \varphi$.

Thus, in all three cases the formula $\varphi_{0}^{(i)}$ satisfies the properties similar to the formula $\varphi^{(j)}$ with respect to the next $(n-l+1)$-th step of the recursion. The formula $\varphi_{0}^{(i)}$ is equivalent to a formula of the following form:

$$
\begin{gathered}
\exists T_{1,1 \ldots} \exists T_{1, \tau_{1}} \exists Y_{l, 1 \ldots}^{(3)} \exists Y_{l, l-1}^{(3)} \exists Y_{l, 1 \ldots} \exists Y_{l, l-1} \exists R \\
\forall T_{2,1 \ldots} \forall T_{2, \tau_{2}} \forall R^{(1)} \forall R^{(4)} \exists T_{3,1 \ldots} \exists T_{3, \tau_{3}} \exists Z_{n-l+1}^{(1)} \\
\exists Y_{l, 1 \ldots}^{(1)} \exists Y_{l, l-1}^{(1)} \exists Z_{n-l+1}^{(2)} \exists Y_{l, 1 \ldots}^{(2)} \exists Y_{l, l-1}^{(2)} \\
\exists Z_{n-l+1}^{(4)} \exists Y_{l, 1 \ldots}^{(4)} \exists Y_{l, l-1}^{(4)}(\psi),
\end{gathered}
$$

where $\psi$ is a certain quantifier-free formula, the conjunction of the quantifierfree parts of the formula $\varphi^{(j)}, \varphi$, and the suitable formula specified above. Indeed, $\varphi_{0}^{(i)}$ has the form of a conjunction of at most three formulas each having the quantifier prefix of the form $\exists \forall \exists$, and because of this, $\varphi_{0}^{(i)}$ is equivalent to a formula with the prefix of the same form $\exists \forall \exists$.

Now we estimate the parameters of formula (16). The number of quantifiers in the first $\exists$-block is at most $\tau_{1}+2 l-1$, in the second $\forall$-block is at most $\tau_{2}+2$, and in the last $\exists$-block is at most $\tau_{3}+3 l$. We conclude by induction on $(n-l)$ that at any step of the recursion a formula of the form (16) with the quantifier prefix $\exists \forall \exists$ in each of three blocks has at most $3 n^{2}$ quantifiers. Thereupon we estimate the number of the atomic subformulae in (16). Denote by $N_{4}$ the number of the atomic subformulae in $\varphi^{(j)}$. Then the number of the atomic subformulae in (16) can be bounded by $N_{4}+O(n)$. Hence by induction on $(n-l)$ we deduce the bound $O((n+p) n)$ on the number of atomic subformulae in a formula of the form (16) on any step of the recursion, taking into account that on the first step the number of atomic subformulae does not exceed $O(p n)$ (see the points $u^{(1)}, \ldots, u^{(p)}$ at the beginning of Section 3 ).

After that we estimate the degrees of the polynomials occurring in formula (16). At the first step of the recursion the formula contains the polynomial $h$, the polynomials from $F[Z]$ determining the points $u^{(1)}, \ldots, u^{(p)}$, and also the element $\mathcal{R}^{2} \in F$. Therefore, for any polynomial $f$ occurring in the latter formula we have the bound $\operatorname{deg}(f) \leq O(D)$ (see the beginning of Section 3), where deg denotes the degree with respect to all the variables, and the bound $\operatorname{deg}_{\delta_{1}, \ldots, \delta_{m}}(f) \leq O\left(D_{0}\right)$. One can easily check that the degrees of the polynomials occurring in (16) in an arbitrary step of the recursion satisfy the similar bounds. 
Now we estimate the degrees of the polynomials

$$
\varphi^{\left(\eta_{n-l}\right)}, \varphi_{j, j_{1}}^{\left(\eta_{1}, \ldots, \eta_{n-l}\right)} \in F[Z]
$$

determining the points $u^{(1)}\left(\eta_{1}, \ldots, \eta_{n-l}\right), \ldots, u^{(q)}\left(\eta_{1}, \ldots, \eta_{n-l}\right)$ given at the beginning of the recursive step in the algorithm. For this purpose, for each $1 \leq j \leq q$, to the family of all the polynomials occurring in the formula $\varphi^{(j)}$, we apply Proposition 3 successively three times (see the Introduction); first with respect to the variables $T_{3,1}, \ldots, T_{3, \tau_{3}}$, second with respect to the variables $T_{2,1}, \ldots, T_{2, \tau_{2}}$ and finally with respect to $T_{1,1}, \ldots, T_{1, \tau_{1}}$. As a result, we get a family of the polynomials

$$
\tilde{g}_{1}, \ldots, \tilde{g}_{\rho} \in F\left[Z_{1}, \ldots, Z_{n-l}, Y_{1}, \ldots, Y_{l}\right]
$$

for which the following bounds are valid: $\rho \leq(n p D)^{O\left(n^{15}\right)}$,

$$
\operatorname{deg}\left(\tilde{g}_{i}\right) \leq(n p D)^{O\left(n^{12}\right)} \text {, and } \operatorname{deg}_{\delta_{1}, \ldots, \delta_{m}}\left(\tilde{g}_{i}\right) \leq D_{0}(n p D)^{O\left(n^{12}\right)},
$$

for $1 \leq i \leq \rho$. The point $u^{(j)}\left(\eta_{1}, \ldots, \eta_{n-l}\right)$ is a null-dimensional connected component (in other words, an isolated point) of a certain $\left\{\tilde{g}_{1}, \ldots, \tilde{g}_{\rho}\right\}$-cell, i.e., an element of the partition $\mathcal{U}\left(\left\{\tilde{g}_{1}, \ldots, \tilde{g}_{\rho}\right\}\right)$ (see Proposition 2). Therefore, there exists a suitable set of indices $I \subset\{1, \ldots, \rho\}$ such that $u^{(j)}\left(\eta_{1}, \ldots, \eta_{n-l}\right)$ is a null-dimensional connected component of the variety $\left\{\sum_{i \in I} \tilde{g}_{i}^{2}=0\right\}$. Applying Proposition 2 to the polynomial $\sum_{i \in I} \tilde{g}_{i}^{2}$ one obtains the bounds:

$$
\begin{gathered}
\operatorname{deg}_{Z}\left(\varphi^{\left(\eta_{n-l}\right)}\right), \operatorname{deg}_{Z}\left(\varphi_{j, j_{1}}^{\left(\eta_{1}, \ldots, \eta_{n-l}\right)} \leq(n p D)^{O\left(n^{13}\right)}\right. \\
\operatorname{deg}_{\delta_{1}, \ldots, \delta_{m}}\left(\varphi^{\left(\eta_{n-l}\right)}\right), \operatorname{deg}_{\delta_{1}, \ldots, \delta_{m}}\left(\varphi_{j_{j}, j_{1}}^{\left(\eta_{1}, \ldots, \eta_{n-l}\right)}\right) \leq D_{0}(n p D)^{O\left(n^{14}\right)} .
\end{gathered}
$$

Applying Proposition 3 to the families of the polynomials from the left sides of systems $(9 \mathrm{a}, \mathrm{b})$ and $(9 \mathrm{~b}, \mathrm{c})$ respectively, the algorithm in Section 3 yields the polynomials $g_{0}^{(i)} \in F\left[T_{2}, \ldots, T_{l}\right]$ for $1 \leq i \leq N_{0}$ and $g_{1}^{(i)} \in F\left[T_{2}, \ldots, T_{l}\right]$ for $1 \leq i \leq N_{1}$, respectively. The following bounds are true:

$$
\begin{aligned}
N_{0}, N_{1} \leq & (n p D)^{O\left(n^{16}\right)} ; \operatorname{deg}_{T_{2}, \ldots, T_{l}}\left(g_{0}^{(i)}\right) ; \operatorname{deg}_{T_{2}, \ldots, T_{l}}\left(g_{1}^{(i)}\right) \leq(n p D)^{O\left(n^{14}\right)} ; \\
& \operatorname{deg}_{\delta_{1}, \ldots, \delta_{m}}\left(g_{0}^{(i)}\right), \operatorname{deg}_{\delta_{1}, \ldots, \delta_{m}}\left(g_{1}^{(i)}\right) \leq D_{0}(n p D)^{O\left(n^{14}\right)} .
\end{aligned}
$$

Hence $\operatorname{deg}_{T_{2}, \ldots, T_{l}}\left(g^{\prime}\right) \leq N^{\prime} \leq(n p D)^{O\left(n^{16}\right)}$. Because of this, the bit lengths of the (integer) entries of the matrix $\mathcal{B}$ are bounded by $l\left(t_{i}\right) \leq O\left(n^{16} \log (n p D)\right)$; thus the bit length of any (integer) entry of the matrix $\overline{\mathcal{M}}^{\left(\eta_{1}, \ldots, \eta_{n-l}\right)}$ does not exceed $O\left(n^{17} \log (n p D)\right)$. Therefore, the length of coefficients $l\left(h^{\left(\eta_{1}, \ldots, \eta_{n-l} l\right)}\right) \leq$ 
$\mathcal{M}+O\left(n^{17} \log (n p D)\right)$ (see the beginning of Section 3). Note that a similar value estimates also the length of the coefficients of every polynomial occurring in formula (16).

Applying again, as above, Proposition 3 three times to formula (16), one obtains the bounds $l\left(\tilde{g}_{i}\right) \leq\left(\mathcal{M}+m D_{0}\right) \mathcal{P}\left((n p D)^{n^{12}}\right)$. Applying again Proposition 2 to the polynomial $\sum_{i \in I} \tilde{g}_{i}^{2}$, one deduces the bounds $l\left(\varphi^{\left(\eta_{n-l}\right)}\right), l\left(\varphi_{j_{j} j_{1}}^{\left(\eta_{1}, \ldots, \eta_{n-l}\right)}\right) \leq$ $\left(\mathcal{M}+m D_{0}\right) \mathcal{P}\left((n p D)^{n^{13}}\right)$.

With the help of Proposition 1 the algorithm finds (see Section 3) all the components, irreducible over the field $F$, of the variety determined by system (12). Proposition 1 implies that the polynomials $g_{1}, \ldots, g_{\tau} \in F\left[Z_{1}, \ldots, Z_{n-l+1}\right.$, $\left.Y_{1}, \ldots, Y_{l-1}\right]$ determining a component $W^{(1)} \subset \bar{F}^{n}$ satisfy $\left(\mathcal{P}\left((n p D)^{n^{14}}\right.\right.$, $D_{0} \mathcal{P}\left((n p D)^{n^{14}}\right),\left(\mathcal{M}+m D_{0}\right) \mathcal{P}\left((n p D)^{n^{14}}\right)$-bounds. Furthermore $\tau \leq$ $\mathcal{P}\left((n p D)^{n^{14}}\right)$.

Thereupon for each component $W^{(1)} \subset \bar{F}^{n}$ which is a curve, and pair $\eta^{(1)}<$ $\eta^{(2)}$ of adjacent options for $\eta_{n-l+1}$, the algorithms finds the connected components of the intersection $W^{(1)} \cap\left\{\eta^{(1)}<Z_{n-l+1}<\eta^{(2)} \wedge Z_{1}=\eta_{1} \wedge \cdots \wedge Z_{n-l}=\right.$ $\left.\eta_{n-1}\right\} \cap \tilde{F}^{n}$ involving Proposition 4. Any such connected component $C_{3}$ (see Section 3 ) is represented by the algorithm as a union of connected semialgebraic curves of the form $\left\{w_{1}>0 \wedge \cdots \wedge w_{\chi_{1}}>0 \wedge w_{\chi_{1}+1} \geq 0 \wedge \cdots \wedge w_{\chi} \geq 0\right\}$. Moreover, the polynomials $w_{i} \in F\left[Z_{1}, \ldots, Z_{n-l+1}, Y_{1}, \ldots, Y_{l-1}\right]$ for $1 \leq i \leq \chi$ satisfy $\left(\mathcal{P}\left((n p D)^{n^{17}}\right), D_{0} \mathcal{P}\left((n p D)^{n^{17}}\right),\left(\mathcal{M}+m D_{0}\right) \mathcal{P}\left((n p D)^{n^{17}}\right)\right)$-bounds. Therefore, these bounds are valid for the representation of the skeleton $\Sigma^{\left(\eta_{1}, \ldots, \eta_{n-l+1}\right)}$ and in particular of the skeleton $\Sigma$.

Now we proceed to the time analysis of the algorithm designed in Section 3. The algorithm has a recursive structure and it can be represented as a rooted tree with depth $n$ in which the branching at the vertex of depth $n-l$ (from the root) corresponding to the values $Z_{1}=\eta_{1}, \ldots, Z_{n-l}=\eta_{n-l}$ is in bijective correspondence with the set of all the options for $\eta_{n-l+1}$. The number of options for $\eta_{n-l+1}$ produced according to the first procedure is at most $\mathcal{P}\left((n p D)^{n^{14}}\right)$ by virtue of Bezout's inequality (see, e.g., Shafarevich 1974), and by taking into account system $(10 a, b)$ and the bounds obtained above on the degrees of $\varphi^{\left(\eta_{i}\right)}$. The number of the options for $\eta_{n-l+1}$ produced according to the third procedure can be bounded by $\mathcal{P}\left((n p D)^{n^{15}}\right.$ ) (see the polynomial $G$ and the variety $\mathcal{W}$ in Section 3). After that, the algorithm, relying on Proposition 2, produces points of the type $u^{(i)}\left(\eta_{1}, \ldots, \eta_{n-l}, \eta_{n-l+1}\right)$ obtained as the intersections of the curves of the kind $W^{(1)} \cap \tilde{F}^{n}$ with the planes $\left\{Z_{1}=\eta_{1} \wedge \cdots \wedge Z_{n-l}=\eta_{n-l} \wedge Z_{n-l+1}=\right.$ $\left.\eta_{n-l+1}\right\}$. The number of points of intersection of curves of the type $W^{(1)}$ with the plane does not exceed the degree of the variety determined by system (12), and hence does not exceed $\mathcal{P}\left((n p D)^{n^{14}}\right)$ because of Bezout's theorem. Thus, 
one can bound the fan-out at each vertex of the tree by $\mathcal{P}\left((n p D)^{n^{15}}\right)$. Therefore, the total number of vertices of tree is at most $\mathcal{P}\left((n p D)^{n^{16}}\right)$.

It suffices to estimate the running time of the recursive step described in Section 3. First, the algorithm looks over all the integer vectors $\left(t_{2}, \ldots, t_{l}\right) \in$ $\left\{0,1, \ldots, N^{\prime}\right\}^{l-1}$ and tests whether the vector $\left(t_{2}, \ldots, t_{l}\right)$ satisfies the conditions from Lemma 9, deciding the solvability of systems $(9 a, b)$ and $(9 b, c)$ (with substituted $\left.t_{2}, \ldots, t_{l}\right)$ using Proposition 2. This requires time $\mathcal{P}\left(\mathcal{M}, D_{0}^{m}\right.$, $\left.(n p D)^{n^{14}(m+1)}\right)$. Thus, taking into account the procedure of looking over the vectors $\left(t_{2}, \ldots, t_{l}\right)$, this stage of the operation of the algorithm takes time $\mathcal{P}(\mathcal{M}$, $\left.D_{0}^{m},(n p D)^{n^{14}\left(n^{3}+m\right)}\right)$. Thereupon the algorithm yields the matrix $\mathcal{M}^{\left(\eta_{1}, \ldots, \eta_{n-1}\right)}$ and the polynomial $h^{\left(\eta_{1}, \ldots, \eta_{n-1}\right)}$ also within the latter time bound. After that the algorithm solves system $(10 a, b)$ using Proposition 2. Similarly, the same time is sufficient for this purpose.

Next, the algorithm considers all $(l-2)$-tuples $0 \leq \lambda_{1}, \ldots, \lambda_{l-2} \leq N_{2}=$ $(2 D)^{l}$, and for each of them finds the irreducible components of the variety determined by system (12), using Proposition 1 , in time $\mathcal{P}\left(\mathcal{M}, D_{0}^{m+n}\right.$, $\left.(n p D)^{n^{14}(n+m)}\right)$. Thereupon the algorithm tests solvability of the quantifierfree formula $(13 a, b)$ with the help of Proposition 2 in the same time.

After that the algorithm produces a representative set of points for the variety $\mathcal{W}$ in the space $(\tilde{F}(\varepsilon))^{n}$, relying on Proposition 2 , in time $\mathcal{P}\left(\mathcal{M}, D_{0}^{m}\right.$, $\left.(n p D)^{n^{15}(m+1)}\right)$. For each of the produced representative points $y^{\prime}$, the algorithm finds its standard part $s t\left(y^{\prime}\right) \in \tilde{F}^{n}$ using Lemma 8 from Grigor'ev \& Vorobjov (1988) within the same time bound. The algorithm tests whether the coordinate function $Z_{n-l+1}$ reaches a local extremum in the point $s t\left(y^{\prime}\right)$ on the curve $W^{(1)} \cap \tilde{F}^{n}$ by applying Proposition 2 to formula (14). For this, time $\mathcal{P}\left(\mathcal{M}, D_{0}^{m},(n p D)^{n^{14}(m+1)}\right)$ suffices.

After that the algorithm applies Proposition 4 to the curve

$$
W^{(1)} \cap\left\{Z_{1}=\eta_{1} \wedge \cdots \wedge Z_{n-l}=\eta_{n-l} \wedge \eta^{(1)}<Z_{n-l+1}<\eta^{(2)}\right\} \cap \tilde{F}^{n}
$$

(for all adjacent options $\eta^{(1)}<\eta^{(2)}$ for $\eta_{n-l+1}$ ) and finds its connected components in time $\mathcal{P}\left(\mathcal{M}, D_{0}^{n+m},(n p D)^{n^{17}(n+m)}\right)$. For each of these connected components $C_{3}$, and the points of the intersection

$$
W_{1} \cap\left\{Z_{1}=\eta_{1} \wedge \cdots \wedge Z_{n-l}=\eta_{n-l} \wedge Z_{n-l+1}=\eta_{n-l+1}\right\} \cap \tilde{F}^{n}
$$

(which are obtained with the aid of Proposition 2, also within the latter time bound), the algorithm finds out which of the obtained points belong to the closure $\bar{C}_{3}$, again using Proposition 2 within the same time-bound. This allows one to construct the skeleton $\Sigma^{\left(\eta_{1}, \ldots, \eta_{n-l}, \eta_{n-l+1}\right)}$ and the corresponding graph 
$F^{\left(\eta_{1}, \ldots, \eta_{n-l}, \eta_{n-l+1}\right)}$ (see the beginning of Section 4). Thus we can summarize as follows the bound on the running time of the algorithm from Section 3 constructing the skeleton $\Sigma$ (and the graph $F$ ), taking into account the bound obtained above on the number of vertices in the tree of the recursion.

LEMMA 14. The algorithm designed in Section 3 constructs a skeleton $\Sigma$ (and the graph $F$ ) for a hypersurface $\{h=0\} \cap \mathcal{D}_{0}(\mathcal{R})$ (having at most one nonsingular point) in time $\mathcal{P}\left(\mathcal{M}, D_{0}^{n+m},(n p D)^{n^{17}(n+m)}\right)$. Furthermore, $\Sigma$ contains the points $u^{(1)}, \ldots, u^{(p)}$. The algorithm represents $\Sigma$ as a union of the connected semialgebraic curves of the type $\left\{w_{1}>0 \wedge \cdots \wedge w_{x_{1}}>\wedge w_{x_{1}+1} \geq 0 \wedge \cdots \wedge w_{x} \geq 0\right\}$, where the polynomials $w_{i} \in F\left[X_{1}, \ldots, X_{n}\right]$ for $1 \leq i \leq x$ satisfy $\left(\mathcal{P}\left((n p D)^{n^{17}}\right.\right.$, $\left.D_{0} \mathcal{P}\left((n p D)^{n^{17}}\right),\left(\mathcal{M}+m D_{0}\right) \mathcal{P}\left((n p D)^{n^{17}}\right)\right)$-bounds, and the graph $F$ specifies which pairs of curves have common endpoints.

Now we turn to complexity bounds of the reduction, as described in Section 2, of the proof of Theorem 1 for the case of system of inequalities (2) (see Section 1) to the case of a bounded nonsingular hypersurface considered in Section 3 (see also Lemma 14). Assume that the polynomials $f_{i} \in \mathbf{Q}_{m}\left[X_{1}, \ldots, X_{n}\right]$ for $1 \leq i \leq k$, occurring in system (2) (see Section 1) satisfy $\left(d, d_{0}, M\right)$-bounds, and also that the points $x$ and $y$ from the semialgebraic set determined by system (2) satisfy $\left(\bar{d}, \bar{d}_{0}, \bar{M}\right)$-bounds. Recall that in Section 2 a polynomial $g_{1} \in Q_{m}\left[\varepsilon_{1}, \varepsilon_{2}\right]\left[X_{1}, X_{n+1}\right]$ was produced which satisfies a $\left(O\left(d_{k}\right), d_{0} \mathcal{P}\left((k d)^{n}\right)\right.$, $\left.\left(M+m \log d_{0}\right) \mathcal{P}\left((k d)^{n}\right)\right)$-bound. In order to prove the first statement of the theorem in the case under consideration, we apply the algorithm designed in Section 3 to the polynomial $h=g_{1}$ and $\mathcal{R}^{2}=R^{2}+\varepsilon_{1}$ (see the beginning of Section 3), where $R$ was produced in Lemma 8 from Section 2 . Therefore, $\mathcal{R}^{2}$ satisfies a $\left(1, d_{0} \mathcal{P}\left((k d)^{n}\right), M \mathcal{P}\left((k d)^{n}\right)\right)$-bound by Lemma 8 . Thus, we construct a skeleton $\Sigma$ and select among the connected components of $\Sigma$ those which lie in the cell $\left\{f_{1}+\varepsilon_{1}>0 \wedge \cdots \wedge f_{k+4}+\varepsilon_{1}>0\right\}$ (the latter is equivalent to the property that an arbitrary point of the connected component belongs to this cell). The number of selected connected components coincides with the number of connected components of the semialgebraic set $V_{0}$ determined by system (2). Therefore the algorithm computes this number in time $\mathcal{P}\left(M, d_{0}^{n+m}\right.$, $\left.(n k d)^{n^{17}(n+m)}\right)$ by virtue of Lemma 14 .

To prove the second statement of Theorem 1, let us assume that $g_{1}^{\prime}$, produced at the end of Section 2, plays the role of $h$ and $\left(R^{\prime}\right)^{2}+\varepsilon_{1}$ plays the role of $\mathcal{R}^{2}$, where $R^{\prime}$ equals to the largest among $R$ and an element $R^{\prime \prime} \in F$ such that the points $x^{\prime}, y^{\prime} \in \mathcal{D}_{0}\left(R^{\prime \prime}\right)$ (see the very end of Section 2). One can easily see that $R^{\prime \prime}$ satisfies a $\left(1, \bar{d}_{0} \mathcal{P}\left((k \bar{d})^{n}\right), \bar{M} \mathcal{P}\left(\left(k \bar{d}^{n}\right)\right)\right.$-bound. Hence $\mathcal{R}^{2}$ satisfies a $\left(1,\left(d_{0}+\bar{d}_{0}\right) \mathcal{P}\left((k \bar{d} \bar{d})^{n}\right),(M+\bar{M}) \mathcal{P}\left((k d \bar{d})^{n}\right)\right)$-bound. Because of this, $g_{1}^{\prime}$ 
satisfies a $\left(O(k d),\left(d_{0}+\bar{d}_{0}\right) \mathcal{P}\left((k d \bar{d})^{n}\right),\left(M+\bar{M}+m \log d_{0}\right) \mathcal{P}\left((k d \bar{d})^{n}\right)\right)$-bound. Furthermore, the algorithm produces the points $x^{(0)}$ and $y^{(0)}$, picking them out from the representative set for the semialgebraic set $U$ (see the end of Section 2), which the algorithm produces using Proposition 2. For this purpose, $\mathcal{P}\left(M, \bar{M},\left(d_{0} \bar{d}_{0}\right)^{m},(k \overline{d d})^{n(m+1)}\right)$ time suffices, and the points $x^{(0)}$ and $y^{(0)}$ satisfy $\left(\mathcal{P}\left((k d \bar{d})^{n}\right),\left(d_{0}+\bar{d}_{0}\right) \mathcal{P}\left((k d \bar{d})^{n},\left(M+\bar{M}+m \log \left(d_{0}+\bar{d}_{0}\right)\right) \mathcal{P}\left((k d \bar{d})^{n}\right)\right)\right.$-bounds. Applying the algorithm designed in Section 3 , we assume that $x^{(0)}$ and $y^{(0)}$ play the role of the points $u^{(1)}$ and $u^{(2)}$. Thus, the algorithm tests whether the points $x$ and $y$ belong to the same connected component of the semialgebraic set $V_{0}$ in time $\mathcal{P}\left(M, \bar{M},\left(d_{0} \bar{d}_{0}\right)^{n+m},(k d \bar{d})^{n^{18}(n+m)}\right)$ by Lemma 14 . This completes the proof of the theorem for the case of system of inequalities (2).

In conclusion let us prove Theorem 1 in the general case (see Section 1). Recall that for an input quantifier-free formula $\Xi$, the algorithm first enumerates all $\left\{f_{1}, \ldots, f_{k}\right\}$-cells using Proposition 2 , and gives a representative set for the partition $\mathcal{U}\left(\left\{f_{1}, \ldots, f_{k}\right\}\right)$. This takes time $\mathcal{P}\left(M, d_{0}^{m+n},(k d)^{n(m+1)}\right)$ and each point from the representative set satisfies a $\left(\mathcal{P}\left((k d)^{n}\right), d_{0} \mathcal{P}\left((k d)^{n}\right)\right.$, $\left.\left.\left(M+m d_{0}\right) \mathcal{P}(k d)^{n}\right)\right)$-bound. Applying the algorithms designed in Sections 2 and 3 to every cell, one can obtain a representative set for the partition $\mathcal{U}\left(\left\{f_{1}, \ldots, f_{k}\right\}\right)$ such that each element of the partition contains exactly one representative point. By what we have shown above, this requires time $\mathcal{P}\left(M, d_{0}^{m+n}\right.$, $\left.(k d)^{n^{19}(n+m)}\right)$.

After that, for every element $V_{1}$ of the partition $\mathcal{U}\left(\left\{f_{1}, \ldots, f_{k}\right\}\right)$, the algorithm tests whether, for the unique connected component $\mathcal{V}_{1}$ of the set $\mathcal{U}_{1}$ such that $\mathcal{V}_{1} \supset V_{1}$ (see Lemma 1 in Section 1), we have $\mathcal{V}_{1} \cap V_{2}^{\left(\varepsilon_{1}, \varepsilon_{2}\right)} \neq \emptyset$. For this we apply Proposition 2 to the semialgebraic set $\mathcal{U}_{1} \cap U_{2}^{\left(\varepsilon_{1}, \varepsilon_{2}\right)}$. It requires time $\mathcal{P}\left(M, d_{0}^{m+n},(k d)^{n(m+1)}\right)$, and each of the produced representative points $x \in \mathcal{U}_{1} \cap U_{2}^{\left(\varepsilon_{1}, \varepsilon_{2}\right)}$ satisfies a $\left(\mathcal{P}\left((k d)^{n}\right), d_{0} \mathcal{P}\left((k d)^{n}\right),\left(M+m d_{0}\right) \mathcal{P}\left((k d)^{n}\right)\right)$-bound. After that one can test whether $x \in V_{2}^{\left(\varepsilon_{1}, \varepsilon_{2}\right)}$ using the algorithms designed in Sections 2 and 3 . This can be done in time $\mathcal{P}\left(M, d_{0}^{n+m},(k d)^{n^{19}(n+m)}\right)$. Consider a point $y$ of the yielded representative set for the partition $\mathcal{U}\left(\left\{f_{1}, \ldots, f_{k}\right\}\right)$ such that $y \in V_{1}$. Again applying the algorithms designed in Sections 2 and 3 , one can find out whether the points $x$ and $y$ lie in the same connected component of the set $\mathcal{U}_{1}$. This also takes time $\mathcal{P}\left(M, d_{0}^{n+m},(k d)^{n^{19}(n+m)}\right)$. This completes the proof of the first statement of the theorem.

To prove the second statement of the theorem (see the very end of Section 1 ), the algorithm, using Proposition 2, finds out in which cells the points $u^{(1)}$ and $u^{(2)}$ lie. For this purpose time $\mathcal{P}\left(M, \bar{M},\left(d_{0} \bar{d}_{0}\right)^{n+m},(k d \bar{d})^{n(m+1)}\right)$ suffices. Thereupon one applies the algorithms designed in Sections 2 and 3 to the cell which contains the point $u^{(1)}$ to specify a connected component of this cell 
which contains the point $u^{(1)}$ (a similar procedure can be done for the point $\left.u^{(2)}\right)$. This requires time $\mathcal{P}\left(M, \bar{M},\left(d_{0} \bar{d}_{0}\right)^{n+m},\left((k d)^{n} \bar{d}\right)^{n^{18}(n+m)}\right)$. The proof of Theorem 1 is now completed.

\section{References}

M. BEN-OR, D. KozEn, AND J. REIF, The complexity of elementary algebra and geometry. J. Comput. System Sci. 32 (1986), 251-264.

J. Canny, The Complexity of Robot Motion Planning. MIT Press, Cambridge, 1988.

J. Canny, D. Yu. Grigor'ev, and N. N. Vorobjov, Jr., Finding connected components of a semialgebraic set in subexponential time. Submitted to Appl. Alg. in Eng. Comm. Comput., 1991.

A. L. Christov and D. Yu. Grigor'ev, Subexponential-time solving systems of algebraic equations, I, II. Preprints LOMI E-9-83 \& E-10-83, Leningrad, 1983.

G. E. Collins, Quantifier elimination for real closed fields by cylindrical algebraic decomposition. Springer Lec. Notes Comp. Sci. 33 (1975), 134-183.

A. DoLd, Lectures on Algebraic Topology. Springer-Verlag, Berlin, 1972.

N. Fitchas, A. Galligo, and J. Morgenstern, Algorithmes rapides en séquentiel et en parallèle pour l'élimination de quantificateurs en géométrie élémentaire. UER de Mathématiques Université de Paris VII, 1988.

D. YU. Grigor'ev, Computational complexity in polynomial algebra. Proc. International Congress of Mathematicians, Berkeley, 1986, 1452-1460.

D. Yu. Grigor'ev, Complexity of deciding Tarski algebra. J. Symb. Comp. 5 (1988), 65-108.

D. Yu. Grigor'ev, J. Heintz, M.-F. Roy, P. Solernó, and N. N. Vorobjov, $\mathrm{J}_{\mathrm{R}}$, Comptage des composantes connexes d'un ensemble semi-algebrique en temps simplement exponential. C. R. Acad. des Sci. Paris, Ser. I 311 (1990), 879-882.

D. YU. Grigor'EV and N. N. Vorobjov, JR., Solving systems of polynomial inequalities in subexponential time. J. Symb. Comp. 5 (1988), 37-64.

J. Heintz, Definability and fast quantifier elimination in algebraically closed field. Theor. Comp. Sci. 24 (1983), 239-278.

J. Heintz, M.-F. Roy, And P. Solernó, Construction de chemins dans un ensemble semi-algébrique. Preprint, Univ. Buenos Aires, Argentina, 1990a.

J. Heintz, M.-F. RoY, AND P. Solernó, Single exponential path finding in semialgebraic sets. Proc. AAECC Conf., Tokyo, 1990b.

J. Heintz, M.-F. RoY, And P. Solernó, Sur la complexité du principe de TarskiSeidenberg. Bull. Soc. Math. France 118 (1990c), 101-126. 
M. W. Hirsch, Differential Topology. Springer-Verlag, Berlin, 1976.

S. LANG, Algebra. Addison-Wesley, New York, 1965.

J. RENEGAR, On the computational complexity and geometry of the first-order the ory of reals, Parts I-III. Technical Report, Cornell University, 1989.

I. R. Shafarevich, Basic Algebraic Geometry. Springer-Verlag, Berlin, 1974. A. TARSKI, A Decision Method for Elementary Algebra and Geometry. University of California Press, 1951.

N. N. Vorobjov, JR., Deciding consistency of systems of inequalities being poly. nomial in exponential functions in subexponential times. Notes of Sci. Seminars of Leningrad Department of Math. Steklov Inst. 176 (1989), 3-52 (in Russian).

N. N. Vorobjov, JR. AND D. YU. Grigor'Ev, Determination of the number of connected components of a semialgebraic set in subexponential time. Soviet Math. Dokl. 42 (1991), 563-566.

H. R. WÜTHRICH, Ein Entscheidungsverfahren für die Theorie der reell-abgeschlossenen Körper. Springer Lec. Notes Comp. Sci. 43 (1976), 138-162.

Manuscript received 17 May, 1990

D. YU. GRIGOR'EV

N. N. VOROBJOV, JR.

V. A. Steklov Mathematical Institute

Academy of Sciences

Fontanka Embankment 27

St. Petersburg, Russia 191011

Current address of GRIGor'Ev:

Department of Computer Science

Pennsylvania State University

State College, PA 16802

USA 